\title{
Geometrical/Physical Interpretation of the Conserved Quantities Corresponding to Noether Symmetries of Plane Symmetric Space-Times
}

\author{
Bismah Jamil, ${ }^{1}$ Tooba Feroze, ${ }^{1}$ and Andrés Vargas ${ }^{2}$ \\ ${ }^{1}$ School of Natural Sciences, National University of Sciences and Technology, Sector H-12, Islamabad, Pakistan \\ ${ }^{2}$ Departamento de Matemáticas, Facultad de Ciencias, Pontificia Universidad Javeriana, Cra. 7 N. 40-62, Bogotá, Colombia \\ Correspondence should be addressed to Bismah Jamil; bismah.jamil@gmail.com
}

Received 23 February 2017; Revised 26 April 2017; Accepted 21 May 2017; Published 30 August 2017

Academic Editor: Igor L. Freire

Copyright (C) 2017 Bismah Jamil et al. This is an open access article distributed under the Creative Commons Attribution License, which permits unrestricted use, distribution, and reproduction in any medium, provided the original work is properly cited.

The aim of this paper is to give the geometrical/physical interpretation of the conserved quantities corresponding to each Noether symmetry of the geodetic Lagrangian of plane symmetric space-times. For this purpose, we present a complete list of plane symmetric nonstatic space-times along with the generators of all Noether symmetries of the geodetic Lagrangian. Additionally, the structure constants of the associated Lie algebras, the Riemann curvature tensors, and the energy-momentum tensors are obtained for each case. It is worth mentioning that the list contains all classes of solutions that have been obtained earlier during the classification of plane symmetric space-times by isometries and homotheties.

\section{Introduction}

Solving a differential equation (DE) by symmetry methods was the original motivation for the development of the classical theory of continuous groups, initiated by S. Lie [1]. Symmetry methods are very useful to construct solutions for a wide class of DEs, especially for nonlinear ones. A very important example of nonlinear partial differential equations (PDEs) from general relativity is the Einstein field equations (EFEs):

$$
R_{i j}-\frac{1}{2} g_{i j} R+g_{i j} \Lambda=\kappa T_{i j}, \quad(i, j=1, \ldots, n)
$$

where $R_{i j}, R, \Lambda$, and $\kappa$ are the Ricci tensor, the Ricci scalar, the cosmological constant, and $\kappa=8 \pi G / c^{4}$, respectively.

Finding general solutions to the EFEs is practically impossible, but various approaches have been employed to find families of solutions to these equations and to classify the corresponding space-times [2]. In this context, symmetries play a fundamental role. They not only provide the solutions to EFEs but also classify the space-times by the symmetries it has. These symmetries appear as inherent geometric symmetries of the set of physically relevant DEs [3-6]. Among the most relevant DEs are the geodesic equations

$$
\ddot{q}^{i}+\Gamma_{j k}^{i} \dot{q}^{j} \dot{q}^{k}=0, \quad(i, j, k=1, \ldots, n)
$$

that describe the trajectories followed by objects moving under the influence of a gravitational field; the study of their symmetries provides insight into the geometric/physical properties of the space-time under consideration.

In the past few decades, a significant amount of relativistic literature has been devoted to the study of symmetries in general relativity [7-24]. In [7], the authors considered Killing vectors (KVs) of spherically symmetric static space-times and concluded that they admit either ten KVs (corresponding to de Sitter, Minkowski, and anti-de Sitter metrics), seven KVs (corresponding to Einstein and anti-Einstein metrics), six KVs (incorporating the Bertotti-Robinson and two other metrics), or only four KVs (the minimal set of isometries). Ahmed and Ziad found the homotheties of spherically symmetric space-times admitting maximal isometry groups larger than $\mathrm{SO}(3)$ along with their metrics, without imposing any restriction on the stress-energy tensor in [12]. Their 
results show that these space-times admit 11, 7, or 5 homotheties. Feroze et al. [13] gave a complete classification of the plane symmetric space-times according to their isometries. They obtained these metrics by solving the Killing equations. The isometries admitted by these metrics are given by the group of motions $G_{r}$ (where $r=3,4,5,6,7$, and 10). Plane symmetric Lorentzian manifolds are classified according to their homothetic vector fields and metrics in [14]. These manifolds can admit 4-, 5-, 7-, and 11-dimensional homothetic Lie algebras.

Lie and Noether symmetries of the geodesic equations in pseudo-Riemannian manifolds have also been studied. For example, they have been computed in terms of the special projective group and homotheties (including KVFs) of the metric in [17]. Tsamparlis derives in [23] the Lie and Noether condition for the equations of motion of a dynamical system in an $n$-dimensional Riemannian space and expresses the symmetry generating vectors in terms of the homothetic and projective collineations of the space.

In this paper, we consider Noether symmetries of the geodetic Lagrangian for general plane symmetric spacetimes. The remaining text is divided into four sections. Section 2 briefly presents basic preliminaries. Section 3 explains the geometry of plane symmetric space-time metrics. Section 4 contains a system of PDEs arising from the Noether symmetry condition (3) of the geodetic Lagrangian for a generic metric of this type (6). After that, a complete list of possible plane symmetric space-times with their corresponding Noether symmetries, conservation laws, and geometric invariants is presented. The physical interpretation of the different conserved quantities that appear is also presented for the most relevant cases. In conclusion, in Section 5 we provide some commentary on the results.

\section{Preliminaries}

The symmetry group of a system of DEs is the largest local group of transformations acting on a manifold $M$ and sending solutions of the system to other solutions. The main feature of a Lie point symmetry of an ordinary differential equation (ODE) is that it is a point transformation in the space of variables, which preserves the set of solutions of the ODE. Explicitly, it will be denoted by $\widetilde{q}^{j}=\widetilde{q}^{j}\left(q^{i} ; \varepsilon\right)$, where the parameter $\varepsilon \in(a, b) \subset \mathbb{R}$ and indices $i, j$ take values from 1 to $n$. These transformations satisfy the axioms of a group under composition. A group of point transformations defines (at least locally) a family of curves in an $n$-dimensional differentiable manifold $M$, which are parameterized by $\varepsilon$ and are called orbits of the group of transformations. These curves may be viewed as the integral curves of a differentiable vector field $\mathbf{X} \in M$ (in this context $\mathbf{X}$ is called an infinitesimal generator and at each point of the manifold $M$ it is tangent to some smooth integral curve $\gamma(\varepsilon) \subset M)$. With the help of these symmetry transformations (or simply symmetries), Lie provided different integration strategies for differential equations [25-27].

Noether symmetries are a special class of Lie point symmetries, associated with a dynamical system described by an action (or variational) principle. If the Lagrangian formulation is given for a dynamical system, one can obtain the conservation laws with the aid of Noether's theorem [28]. In other words, each symmetry determines a conserved quantity. Conservation laws help us to understand and describe a dynamical system.

A generator of an infinitesimal transformation $\mathbf{X}$ is called a Noether symmetry [25] of the dynamical system described by the Lagrangian $L\left(s, q^{i}, \dot{q}^{i}\right)$ if it satisfies

$$
\mathbf{X} L+(\mathbf{D} \xi) L=\mathbf{D} A,
$$

where $A\left(s, q^{i}\right)$ is some gauge term, $\mathbf{D}$ is an operator given by $\mathbf{D}=\partial_{s}+\dot{q}^{i} \partial_{q^{i}}$, and

$$
\begin{aligned}
& \mathbf{X}=\xi\left(s, q^{i}\right) \partial_{s}+\eta^{j}\left(s, q^{i}\right) \partial_{q^{j}}+\dot{\eta}^{j}\left(s, q^{i}, \dot{q}^{i}\right) \partial_{\dot{q}^{j}} \\
&(i, j=1, \ldots, n) .
\end{aligned}
$$

Here $\dot{\eta}^{i}=\eta_{, s}^{i}+\dot{q}^{j} \eta_{, j}^{i}-\dot{q}^{i} \xi_{, s}-\dot{q}^{i} \dot{q}^{j} \xi_{, j}$ is the first-order prolonged part of the generator and $q^{i}$ represents space-time coordinates and $\dot{q}^{i}$ their derivatives with respect to an affine parameter $s$. Corresponding to each Noether symmetry, one can find a conserved quantity

$$
\varphi=\xi\left[\dot{q}^{k} L_{, \dot{q}^{k}}-L\right]-\eta^{k} L_{, \dot{q}^{k}}+A\left(s, q^{i}\right),
$$

satisfying $\mathbf{X} \varphi=0$. Noether symmetries with $A=0$ are called the variational symmetries [29]. Noether symmetries of the geodetic Lagrangian constitute subalgebra of the algebra of Lie symmetries of the geodesic equations. It was shown in [30] that this subalgebra is generated (in the space of variables) by the coordinate vector associated with the affine parameter of the geodesics and the homothetic Lie algebra of the underlying space-time.

Lie algebras come with a skew-symmetric product called a Lie bracket and can be characterized by the so-called structure constants with respect to some basis of the linear space. Explicitly, for a given basis $\left\{\mathbf{X}_{i}\right\}$, the Lie brackets satisfy relations of the form $\left[\mathbf{X}_{i}, \mathbf{X}_{j}\right]=C_{i j}^{k} \mathbf{X}_{k}$, for some appropriate constants $C_{i j}^{k}(i, j, k=1, \ldots, m)$ called structure constants of the Lie algebra. Throughout the text, we exhibit the Lie algebra of symmetries for each case in terms of these structure constants.

There are also important tensorial quantities that describe the geometric and physical properties of each space-time. Let us recall briefly the definition and meaning of those ones that we explicitly calculate for the metrics in the classification. These quantities allow us to identify the space-times as most metrics do not appear in recognizable form due to the use of nonstandard coordinates. Hence, these quantities are important to study all the possible nonstatic plane symmetric cases.

(i) The Riemann curvature tensor $R_{i j k l}$ is defined in local coordinates $\left(q^{i}\right)$ by the noncommutativity of the LeviCivita covariant derivatives $\nabla_{i}$ and $\nabla_{j}$ acting on a coordinate vector $\partial_{k}$; namely, $R_{i j k}^{l} \partial_{l}=\nabla_{[i} \nabla_{j]} \partial_{k}$. In terms of geodesic normal coordinates (adapted to the 
motion of free moving particles in space-time) the Riemann tensor at a point encodes the information of the difference up to second order (as a function of the distance to that point) between the standard Minkowski metric and the space-time metric.

(ii) The Ricci tensor $R_{i j}=R_{i k j}^{k}=g^{k l} R_{l i k j}$ in normal coordinates is proportional to the relative difference up to second order between the volume elements of the Minkowski metric and the space-time metric around a given point. Similarly, the scalar curvature or Ricci scalar $R=R_{i}^{i}=g^{i j} R_{i j}$ is a scalar invariant proportional to the relative difference up to second order between the volume of a Minkowski metric ball and the volume of a space-time metric ball around the point in consideration.

(iii) The stress-energy tensor $T_{i j}$ and its trace $T=T^{i}{ }_{i}$ provide information on the momentum/energy content of the given space-time coming from the presence of particles or fields in it. In the EFEs the components of this tensor are the source of space-time curvature [31].

\section{Geometry of Plane Symmetric Nonstatic Space-Times}

A plane symmetric space-time is a Lorentzian manifold possessing a physical stress-energy tensor and some symmetry properties that reduce the general metric of the manifold to the form [2]

$$
d s^{2}=e^{2 \gamma(t, x)} d t^{2}-e^{2 \lambda(t, x)} d x^{2}-e^{2 \mu(t, x)}\left(d y^{2}+d z^{2}\right) .
$$

Plane symmetric metrics admit the Lie group $\mathrm{SO}(2) \ltimes \mathbb{R}^{2}$ as the minimal set of isometries in such a way that group orbits are two-dimensional hypersurfaces of zero curvature. The minimal set of Noether symmetries for these space-times is

$$
\begin{aligned}
& \mathbf{X}_{1}=z \partial_{y}-y \partial_{z}, \\
& \mathbf{X}_{2}=\partial_{z} \\
& \mathbf{X}_{3}=\partial_{y} \\
& \mathbf{Y}_{0}=\partial_{s}
\end{aligned}
$$

that is, all metrics listed in this paper admit this minimal set. The nonvanishing components of the geometrical/physical quantities previously described which are associated with a space-time metric of form (6) are as follows:

$R_{i j k l}$

$$
\begin{aligned}
& R_{0101}=e^{2 \lambda}\left(\ddot{\lambda}+\dot{\lambda}^{2}-\dot{\nu} \dot{\lambda}\right)-e^{2 \nu}\left(\nu^{\prime \prime}+\nu^{\prime 2}-\nu^{\prime} \lambda^{\prime}\right), \\
& R_{0202}=R_{0303}=e^{2 \mu}\left(\ddot{\mu}+\dot{\mu}^{2}-\dot{\nu} \dot{\mu}\right)-e^{2(\mu+\nu-\lambda)} \nu^{\prime} \mu^{\prime},
\end{aligned}
$$

$$
\begin{aligned}
& R_{0212}=R_{0313}=e^{2 \mu}\left(\dot{\mu}^{\prime}+\dot{\mu} \mu^{\prime}-\nu^{\prime} \dot{\mu}-\dot{\lambda} \mu^{\prime}\right), \\
& R_{1212}=R_{1313}=e^{2 \mu}\left(\mu^{\prime \prime}+\mu^{\prime 2}-\lambda^{\prime} \mu^{\prime}\right)-e^{2(\mu-\nu+\lambda)} \dot{\lambda} \dot{\mu}, \\
& R_{2323}=\mu^{\prime 2} e^{2(2 \mu-\lambda)}-\dot{\mu}^{2} e^{2(2 \mu-\nu)} .
\end{aligned}
$$

$R_{i j}$

$$
\begin{aligned}
R_{00}= & e^{2(\nu-\lambda)}\left(\nu^{\prime \prime}+\nu^{\prime 2}-\nu^{\prime} \lambda^{\prime}+2 \nu^{\prime} \mu^{\prime}\right) \\
& -\left(\ddot{\lambda}+\dot{\lambda}^{2}-\dot{\nu} \dot{\lambda}+2 \ddot{\mu}+2 \dot{\mu}^{2}-2 \dot{\nu} \dot{\mu}\right), \\
R_{01}= & -2\left(\dot{\mu}^{\prime}+\dot{\mu} \mu^{\prime}-\nu^{\prime} \dot{\mu}-\dot{\lambda} \mu^{\prime}\right), \\
R_{11}= & e^{2(\lambda-\nu)}\left(\ddot{\lambda}+\dot{\lambda}^{2}-\dot{v} \dot{\lambda}+2 \dot{\lambda} \dot{\mu}\right) \\
& -\left(\nu^{\prime \prime}+\nu^{\prime 2}-\nu^{\prime} \lambda^{\prime}+2 \mu^{\prime \prime}+2 \mu^{\prime 2}-2 \lambda^{\prime} \mu^{\prime}\right), \\
R_{22}= & R_{33} \\
= & e^{2(\mu-\nu)}\left(\ddot{\mu}+2 \dot{\mu}^{2}-\dot{v} \dot{\mu}+\dot{\lambda} \dot{\mu}\right) \\
& -e^{2(\mu-\lambda)}\left(\mu^{\prime \prime}+2 \mu^{\prime 2}+\nu^{\prime} \mu^{\prime}-\lambda^{\prime} \mu^{\prime}\right) .
\end{aligned}
$$

$R_{i}^{i}$

$$
\begin{aligned}
R & =2 e^{-2 \lambda}\left(2 \mu^{\prime \prime}+3 \mu^{\prime 2}+2 \nu^{\prime} \mu^{\prime}-2 \lambda^{\prime} \mu^{\prime}+\nu^{\prime \prime}+\nu^{\prime 2}\right. \\
& \left.-\nu^{\prime} \lambda^{\prime}\right)-2 e^{-2 \nu}\left(2 \ddot{\mu}+3 \dot{\mu}^{2}-2 \dot{\nu} \dot{\mu}+2 \dot{\lambda} \dot{\mu}+\ddot{\lambda}+\dot{\lambda}^{2}\right. \\
& -\dot{\nu} \dot{\lambda}) .
\end{aligned}
$$

$T_{i j}$

$$
\begin{aligned}
\kappa T_{00} & =e^{2(\nu-\lambda)}\left(2 \lambda^{\prime} \mu^{\prime}-2 \mu^{\prime \prime}-3 \mu^{\prime 2}\right)+\left(\dot{\mu}^{2}+2 \dot{\lambda} \dot{\mu}\right), \\
\kappa T_{01} & =R_{01}, \\
\kappa T_{11} & =e^{2(\lambda-\nu)}\left(2 \dot{\nu} \dot{\mu}-2 \ddot{\mu}-3 \dot{\mu}^{2}\right)+\left(\mu^{\prime 2}+2 \nu^{\prime} \mu^{\prime}\right), \\
\kappa T_{22} & =\kappa T_{33} \\
= & e^{2(\mu-\lambda)}\left(\mu^{\prime \prime}+\mu^{\prime 2}+\nu^{\prime} \mu^{\prime}-\lambda^{\prime} \mu^{\prime}+\nu^{\prime \prime}+\nu^{\prime 2}-\nu^{\prime} \lambda^{\prime}\right) \\
& -e^{2(\mu-\nu)}\left(\ddot{\mu}+\dot{\mu}^{2}-\dot{v} \dot{\mu}+\dot{\lambda} \dot{\mu}+\ddot{\lambda}+\dot{\lambda}^{2}-\dot{\nu} \dot{\lambda}\right) .
\end{aligned}
$$

$$
T_{i}^{i}
$$

$$
\begin{aligned}
T & =-2 e^{-2 \lambda}\left(2 \mu^{\prime \prime}+3 \mu^{\prime 2}+2 \nu^{\prime} \mu^{\prime}-2 \lambda^{\prime} \mu^{\prime}+\nu^{\prime \prime}+\nu^{\prime 2}\right. \\
& \left.-\nu^{\prime} \lambda^{\prime}\right)+2 e^{-2 \nu}\left(2 \ddot{\mu}+3 \dot{\mu}^{2}-2 \dot{\nu} \dot{\mu}+2 \dot{\lambda} \dot{\mu}+\ddot{\lambda}+\dot{\lambda}^{2}\right. \\
& -\dot{\nu} \dot{\lambda}) .
\end{aligned}
$$

Here, the stress-energy tensor $T_{i j}$ is obtained by assuming the validity of EFEs (1) with cosmological constant $\Lambda=0$. 
In the list of Noether symmetries associated with the geodetic Lagrangian for each metric we find two different categories of vector fields, one containing only geometric collineations of space-time alone and the other containing symmetries that can be interpreted as collineations in the extended space of the all the variables $\left(s, q^{i}\right)$ involved in the geodesic equations (2).

Noether symmetries that are collineations of the spacetime will be denoted by $\mathbf{X}_{i}$ and all of them correspond to Killing vector fields (KVFs); that is, they are infinitesimal generators of isometries or, equivalently, their flow preserves the metric on $M$. For each of these generators it is always possible to find an appropriate coordinate frame, called adapted frame, such that the vector field $\mathbf{X}_{i}$ points in the direction of one of the coordinate basis vectors. This means that $\mathbf{X}_{i}$ takes the form $\partial_{u}:=\partial / \partial u$ of an infinitesimal generator of translations along the $u$-coordinate direction. Moreover, by Noether's theorem the conservation law arising from this symmetry can be interpreted as the conservation of generalized momentum associated with that particular $u$-direction. For example, for the Minkowski metric linear momentum conservation arises from space translations, conservation of angular momentum arises from rotations (translations in angular directions), and conservation of energy arises from translations in the time coordinate.

For the second category of Noether symmetries we use the notation $\mathbf{Y}_{i}$. In general, these symmetries are associated with the affine parameter $s$ of the geodesics and they cannot be interpreted as space-time collineations. Nevertheless, extending space-time and its metric in an appropriate way to the whole space of variables, which locally takes the form $\left(s, q^{i}\right)$, those symmetries can still be interpreted as collineations of this extended space. Here we consider the simple extension of the metric given by $g_{r s}=g_{s r}=\delta_{r s}$, where $r \in\left\{s, q^{i}\right\}$ and $s$ is the affine parameter.

(i) The first family of these symmetries can be interpreted as KVFs of the extended space; they can take the forms $\partial_{s}$ and $s \partial_{i}$. The conserved quantity $\varphi\left(s, q^{i}, \dot{q}^{i}\right)$ corresponding to $\partial_{s}$ is the geodetic Lagrangian $L$ (which does not depend explicitly on $s$ ). The one corresponding to $s \partial_{i}$ for spatial $i$-components is the invariant $\varphi\left(s, q^{i}, \dot{q}^{i}\right)=q^{i}-s \dot{q}^{i}$ and it corresponds to the uniform motion of the center of mass along the $q^{i}$ coordinate. Finally the symmetry $s \partial_{t}$ has $\varphi(s, t, \dot{t})=$ $t-s \dot{t}$ as invariant quantity and it represents the retarded time arising from a change of center of mass reference frame to one at rest with respect to the coordinate system chosen.

(ii) The second family of these symmetries correspond to homothetic vector fields (HVFs) of the extended space of variables. They take the general form $s \partial_{s}+$ $q^{i} \partial_{i}$, where, depending on the particular metric, the sum on the repeated index $i$ may be needed on all or some (even only one) of its values. These proper HVFs arise from the scaling invariance of the geodesic equations. To see how they are derived from the action of the group of dilations on the extended space $\left(s, q^{i}\right)$, suppose that $\Phi_{\lambda}(s)=\lambda(s, \gamma(s))$ represents a uniparametric family of dilations acting on a given geodesic $(s, \gamma(s))$ and indexed by a continuous scaling parameter $\lambda>0$. Then, the infinitesimal action along the points on the geodesics is given by $\mathbf{Y}_{\mathrm{HVF}}=$ $\left.(\partial / \partial \lambda) \Phi_{\lambda}(s)\right|_{\lambda=1}=(s, \gamma(s))$. With respect to the local coordinate basis $\left(\partial_{s}, \partial_{i}\right)$ it becomes $\mathbf{Y}_{\mathrm{HVF}}=s \partial_{s}+q^{i} \partial_{i}$.

(iii) Finally, the last group of Noether symmetries in this category corresponds to a particular class of projective vector fields (PVFs) on the extended space. They are of the form $s^{2} \partial_{s}+s q^{i} \partial_{i}$, where again the sum on $i$ may appear on all or some of its values. These proper PVFs arise from a fractional linear transformation of the affine parameter of a geodesic. Explicitly, for a continuous $\alpha>0$ let

$$
\Phi_{\alpha}(s)=\left(\frac{s}{1-\alpha s}, \frac{\gamma(s)}{1-\alpha s}\right)
$$

represent a uniparametric family of projective transformations acting on a geodesic $(s, \gamma(s))=\Phi_{0}(s)$. It is important to note that these transformations take the trajectory defined by $\gamma$ to itself. In this case, the infinitesimal generator along $\gamma(s)$ is given by $\mathbf{Y}_{\mathrm{PVF}}=$ $\left.(\partial / \partial \alpha) \Phi_{\alpha}(s)\right|_{\alpha=0}=s(s, \gamma(s))$. With respect to the local coordinate basis $\left(\partial_{s}, \partial_{i}\right)$ it becomes $\mathbf{Y}_{\mathrm{PVF}}=s^{2} \partial_{s}+s q^{i} \partial_{i}$.

We remark that, in general, proper PVFs of the spacetime should not appear as Noether symmetries of the affine geodesic equations. But the flows of the PVFs of the form $\mathbf{Y}_{\text {PVF }}$ just explained send each geodesic to itself changing only its affine parameter from $s$ to $\widetilde{s}=s /(1-\alpha s)$. The transformed points on a geodesic have the form $(\widetilde{s}, \gamma(\widetilde{s}))$, where $\gamma(\widetilde{s})$ satisfies again the affine geodesic equations in terms of $\widetilde{s}$. This is why infinitesimal generators of this type do appear as Noether symmetries, but only when considering the extended space $\left(s, q^{i}\right)$.

\section{Noether Symmetries of the Geodetic Lagrangian of Plane Symmetric Nonstatic Space-Times}

Our aim is to find Noether symmetries for all geodetic Lagrangians of plane symmetric nonstatic space-times. Therefore, we consider the geodetic Lagrangian associated with the space-time (6); that is,

$$
L=e^{2 v(t, x)} \dot{t}^{2}-e^{2 \lambda(t, x)} \dot{x}^{2}-e^{2 \mu(t, x)}\left(\dot{y}^{2}+\dot{z}^{2}\right) .
$$

Following the procedure adopted in [9], we find that the Noether symmetry condition (3) for Lagrangian (14) splits into the following system of PDEs:

$$
\begin{aligned}
\xi_{, t} & =\xi_{, x}=\xi_{, y}=\xi_{, z}=A_{, s}=0, \\
2 \dot{\nu} \eta^{0}+2 v^{\prime} \eta^{1}+2 \eta_{, t}^{0} & =\xi_{, s},
\end{aligned}
$$


TABLE 1: Conserved quantities corresponding to each Noether symmetry.

\begin{tabular}{ll}
\hline Generator; gauge term: $A\left(s, q^{i}\right)$ & Conserved quantity: $\varphi\left(s, q^{i}, \dot{q}^{i}\right)$ \\
\hline $\mathbf{X}_{0}=\partial_{t}$ & $-2 \dot{t}$ \\
$\mathbf{X}_{1}=z \partial_{y}-y \partial_{z}$ & $2(z \dot{y}-y \dot{z})$ \\
$\mathbf{X}_{2}=\partial_{z}$ & $2 \dot{z}$ \\
$\mathbf{X}_{3}=\partial_{y}$ & $2 \dot{y}$ \\
$\mathbf{X}_{4}=x \partial_{t}+t \partial_{x}$ & $2(x \dot{t}-t \dot{x})$ \\
$\mathbf{X}_{5}=\partial_{x}$ & $2 \dot{x}$ \\
$\mathbf{X}_{6}=z \partial_{x}-x \partial_{z}$ & $2(z \dot{x}-x \dot{z})$ \\
$\mathbf{X}_{7}=z \partial_{t}+t \partial_{z}$ & $2(z \dot{t}-t \dot{z})$ \\
$\mathbf{X}_{8}=y \partial_{x}-x \partial_{y}$ & $2(y \dot{x}-x \dot{y})$ \\
$\mathbf{X}_{9}=y \partial_{t}+t \partial_{y}$ & $2(y \dot{t}-t \dot{y})$ \\
$\mathbf{Y}_{0}=\partial_{s}$ & $L$ \\
$\mathbf{Y}_{1}=s \partial_{y} ; A_{1}=-2 y$ & $2(y-s \dot{y})$ \\
$\mathbf{Y}_{2}=s \partial_{z} ; A_{2}=-2 z$ & $2(z-s \dot{z})$ \\
$\mathbf{Y}_{3}=\frac{1}{2}\left(s^{2} \partial_{s}+s t \partial_{t}+s x \partial_{x}+s y \partial_{y}+s z \partial_{z}\right) ; A_{3}=\frac{1}{2}\left(t^{2}-x^{2}-y^{2}-z^{2}\right)$ & 1 \\
$\mathbf{Y}_{4}=s \partial_{t} ; A_{4}=2 t$ & 2 \\
$\mathbf{Y}_{5}=s \partial_{x} ; A_{5}=-2 x$ & $2(t-s \dot{t}-x \dot{x}-y \dot{y}-z \dot{z})+\frac{1}{2}\left(t^{2}-x^{2}-y^{2}-z^{2}\right)$ \\
$\mathbf{Y}_{6}=s \partial_{s}+\frac{1}{2}\left(t \partial_{t}+x \partial_{x}+y \partial_{y}+z \partial_{z}\right)$ & $2(x-s \dot{x})$ \\
\hline
\end{tabular}

$$
\begin{aligned}
2 \dot{\lambda} \eta^{0}+2 \lambda^{\prime} \eta^{1}+2 \eta_{, x}^{1} & =\xi_{, s}, \\
2 \dot{\mu} \eta^{0}+2 \mu^{\prime} \eta^{1}+2 \eta_{, y}^{2} & =\xi_{, s}, \\
2 \dot{\mu} \eta^{0}+2 \mu^{\prime} \eta^{1}+2 \eta_{, z}^{3} & =\xi_{, s}, \\
e^{2 \lambda(t, x)} \eta_{, y}^{1}+e^{2 \mu(t, x)} \eta_{, x}^{2} & =0, \\
e^{2 \lambda(t, x)} \eta_{, z}^{1}+e^{2 \mu(t, x)} \eta_{, x}^{3} & =0, \\
\eta_{, z}^{2}+\eta_{, y}^{3} & =0, \\
e^{2 \nu(t, x)} \eta_{, x}^{0} & =e^{2 \lambda(t, x)} \eta_{, t}^{1}, \\
e^{2 \nu(t, x)} \eta_{, y}^{0} & =e^{2 \mu(t, x)} \eta_{, t}^{2}, \\
e^{2 \nu(t, x)} \eta_{, z}^{0} & =e^{2 \mu(t, x)} \eta_{, t}^{3}, \\
2 e^{2 \gamma(t, x)} \eta_{, s}^{0} & =A_{, t}, \\
-2 e^{2 \lambda(t, x)} \eta_{, s}^{1} & =A_{, x}, \\
-2 e^{2 \mu(t, x)} \eta_{, s}^{2} & =A_{, y}, \\
-2 e^{2 \mu(t, x)} \eta_{, s}^{3} & =A_{, z},
\end{aligned}
$$

where "." and ", " represent derivatives with respect to the coordinates $t$ and $x$, respectively. Here $\xi, A$, and $\eta^{i}(i=$ $0,1,2,3)$ are functions depending on $s$ and $q^{i}$, where $q^{i}$ stands for $t, x, y$, and $z$. The set of solutions to this system of equations provides all possible metrics admitting a higher (or equal) number of symmetries compared to the minimal symmetry group along with their Noether symmetries. These solutions comprise all the existing results listed in [13, 32-34]. A complete list of metrics along with their Noether symmetry generators, structure constants of the corresponding Lie algebras, and the relevant associated tensors defined in the previous section is provided here. The list is organized by decreasing number of symmetries.

4.1. Seventeen Noether Symmetries. Our list begins with the standard constant Lorentzian metric that admits the maximum number of symmetries. We provide for this case a detailed interpretation of all the conserved quantities. Similar symmetries and associated quantities appear later for other metrics and their interpretation is analogous to the one given in this case.

Minkowski Metric. The geodetic Lagrangian of the well known Minkowski space-time metric

$$
d s^{2}=d t^{2}-d x^{2}-d y^{2}-d z^{2}
$$

admits seventeen Noether symmetries [33, 35]. The Noether symmetry generators along with their gauge terms denoted by $A_{i}(i=1, \ldots, 5)$ and their associated conserved quantities are given in Table 1.

The structure constants of the Lie algebra generated by all these symmetries are

$$
\begin{aligned}
& C_{13}^{2}=C_{04}^{5}=C_{07}^{2}=C_{09}^{3}=C_{13}^{2}=C_{18}^{6}=C_{19}^{7}=C_{27}^{0} \\
& =C_{89}^{4}=C_{39}^{0}=C_{\widehat{0} \hat{3}}^{\widehat{4}}=C_{1 \widehat{1}}^{\hat{2}}=C_{6 \widehat{6}}^{\widehat{2}}=C_{8 \widehat{6}}^{\hat{1}}=C_{46}^{7} \\
& =C_{48}^{9}=C_{56}^{2}=C_{58}^{3}=C_{67}^{4}=C_{79}^{1}=1 \text {, } \\
& C_{27}^{0}=C_{39}^{0}=C_{\widehat{0} \hat{1}}^{3}=C_{\widehat{0} \widehat{2}}^{2}=C_{\widehat{04}}^{0}=C_{\widehat{0} \widehat{5}}^{0}=C_{\widehat{0} \widehat{6}}^{5}=1 \text {, }
\end{aligned}
$$




$$
\begin{aligned}
& C_{\widehat{1} \widehat{4}}^{\hat{1}}=C_{2 \widehat{3}}^{\hat{2}}=-\frac{1}{2}, \\
& C_{0 \widehat{3}}^{\widehat{5}}=C_{5 \widehat{3}}^{\widehat{6}}=C_{0 \widehat{4}}^{0}=C_{2 \widehat{4}}^{2}=C_{3 \widehat{4}}^{3}=C_{5 \widehat{4}}^{5}=C_{\widehat{4} \widehat{5}}^{0}=C_{\widehat{4} \hat{6}}^{5} \\
& =\frac{1}{2} \text {, } \\
& C_{12}^{3}=C_{8 \hat{1}}^{\widehat{6}}=C_{9 \hat{1}}^{\widehat{5}}=C_{\widehat{1} \hat{2}}^{\widehat{1}}=C_{\widehat{6} \hat{2}}^{\widehat{6}}=C_{7 \widehat{2}}^{\hat{5}}=C_{\widehat{3} \hat{4}}^{\widehat{3}}=C_{4 \widehat{5}}^{\widehat{6}} \\
& =C_{7 \widehat{5}}^{\widehat{2}}=C_{9 \widehat{5}}^{\widehat{1}}=C_{4 \widehat{6}}^{\widehat{5}}=C_{12}^{3}=C_{16}^{8}=C_{17}^{9}=C_{26}^{5} \\
& =C_{38}^{5}=C_{45}^{0}=C_{47}^{6}=C_{49}^{8}=C_{68}^{1}=-1 \text {. }
\end{aligned}
$$

Here, the hat on a superscript (or subscript) index $\hat{i}$ refers to components associated with elements of the basis given by symmetries of type $\mathbf{Y}_{i}$. The Noether symmetries denoted by $\mathbf{X}_{i}$ correspond to isometry generators and the ones denoted by $\mathbf{Y}_{j}$ correspond to proper (nonisometry) generators. It is important to observe that all nonisometry symmetry generators $\mathbf{Y}_{j}$ are dependent on the affine parameter $s$.

Let us now present a brief discussion of the geometri$\mathrm{cal} /$ physical interpretation of these generators. The Noether symmetry generated by $\mathbf{X}_{0}$ corresponds to time translation, this is an isometry of the space-time, and it is well known that the associated conservation law is the conservation of energy. The generators $\mathbf{X}_{2}, \mathbf{X}_{3}$, and $\mathbf{X}_{5}$ represent spatial translations and correspond to the conservation of linear momentum in the $z$-, $y$-, and $x$-direction, respectively. The generators $\mathbf{X}_{1}$, $\mathbf{X}_{6}$, and $\mathbf{X}_{8}$ correspond to spatial rotations with respect to the $x$-, $y$-, and $z$-axes, respectively, and their presence imply the conservation of angular momentum around these axes. The Noether symmetries $\mathbf{X}_{4}, \mathbf{X}_{7}$, and $\mathbf{X}_{9}$ can be interpreted as "hyperbolic rotations" and correspond physically to the change of reference frame given by Lorentz boosts. The conserved quantities associated with these symmetries are similar to the expressions for the angular momentum. This is not surprising given that boosts are a special kind of rotations of the underlying Lorentzian coordinates involving the time $t$ [36].

The generator $\mathbf{Y}_{0}$ is a symmetry arising from the invariance of the geodetic Lagrangian under translations of the affine parameter $s$. In other words, it corresponds to the freedom in the choice of origin for this parameter. The Noether symmetries associated with $\mathbf{Y}_{1}, \mathbf{Y}_{2}$, and $\mathbf{Y}_{5}$ (neither isometries nor homotheties of space-time alone) correspond to Galilean symmetries. The Galilean principle stating that all reference frames that move with constant velocity relative to each other are equivalent is also a symmetry principle. In other words, the Galilean symmetry represents the change of reference frame to one moving along some geodesic (center of mass frame) in a particular direction. Similarly, $\mathbf{Y}_{4}$ is neither an isometry nor homothety of space-time but represents the retarded time between an observer moving with constant velocity along the geodesic (center of mass frame) and a fixed observer static at the origin of the geodesic. In other words, the difference between the time measured by two observers (one fixed and one moving with constant velocity).
It is important to note that these four generators $\mathbf{Y}_{1}, \mathbf{Y}_{2}, \mathbf{Y}_{4}$, and $\mathbf{Y}_{5}$ can be interpreted geometrically as Killings of the parameter space $\mathbb{R} \times M$, represented locally by the coordinates $(s, t, x, y, z)$ and assuming that the metric has been extended to this space by taking the product of the Euclidean metric of $\mathbb{R}$ with the Lorentzian metric of the space-time $M$. From this perspective, it is possible to say that the generator $\mathbf{Y}_{3}$ is not homothety nor a projective symmetry of space-time (i.e., not a pure geometric symmetry of the underlying Lorentzian manifold $M$ ), but it does correspond to a projective symmetry of the solutions in the parameter space $\mathbb{R} \times M$ endowed with this extended metric. As it was explained in the previous section, this symmetry preserves geodesic trajectories but not their affine parameter. After composition of any solution with the flow of this symmetry, the trajectory of each geodesic is sent to itself, but the parametrization will not necessarily be affine anymore. Similarly, the generator $\mathbf{Y}_{6}$ can be interpreted as homothety of the extended space $(s, t, x, y, z)$ that corresponds physically to the scaling invariance of the geodesic equations. Indeed, if we scale the affine parameter $s$ along the geodesic, then we have to scale each coordinate in their respective directions to preserve the solution. Therefore, under this scaling symmetry, the solution space remains invariant.

Since Minkowski space-time is a flat space-time, all the components of the Riemann curvature tensor $R_{i j k l}$, the Ricci tensor $R_{i j}$, and the stress-energy tensor $T_{i j}$ and their traces vanish.

Milne Metric. The Milne metric is a special case of a Friedmann-Lemaitre-Robertson-Walker (FLRW) model in the limit of zero energy density (empty universe). It illustrates some features that have been associated with an expanding universe. It admits seventeen Noether symmetries and all the components of the Riemann curvature tensor vanish; therefore, it is locally flat [37]. In other words, the Milne metric is a simple reparametrization of flat Minkowski space. The form of the metric is given by

$$
d s^{2}=\left(\frac{x}{a}\right)^{2} d t^{2}-d x^{2}-e^{2 t / a}\left(\frac{x}{a}\right)^{2}\left(d y^{2}+d z^{2}\right),
$$

and its nonstatic analogue is

$$
d s^{2}=d t^{2}-\left(\frac{t}{a}\right)^{2} d x^{2}-e^{2 x / a}\left(\frac{t}{a}\right)^{2}\left(d y^{2}+d z^{2}\right),
$$

$(a \neq 0)$.

4.2. Eleven Noether Symmetries. The metrics admitting eleven Noether symmetries are variants of de Sitter and anti-de Sitter space-times.

de Sitter Space-Times. The following metric is a variant of de Sitter space-time in flat coordinates (with an appropriate change of variables it is possible to transform the metric into the original form of the de Sitter space-time). It is a maximally symmetric Lorentzian manifold with constant 
TABLE 2: Conserved quantities corresponding to each Noether symmetry.

Generator
$\mathbf{X}_{0}=\partial_{x}$
$\mathbf{X}_{4}=y \partial_{x}-x \partial_{y}$
$\mathbf{X}_{5}=z \partial_{x}-x \partial_{z}$
$\mathbf{X}_{6}=\partial_{t}-\frac{1}{a}\left(x \partial_{x}+y \partial_{y}+z \partial_{z}\right)$
$\mathbf{X}_{7}=z \partial_{t}-\frac{z}{a}\left(x \partial_{x}+y \partial_{y}\right)+\frac{1}{2 a}\left(-a^{2} e^{-2 t / a}+x^{2}+y^{2}-z^{2}\right) \partial_{z}$
$\mathbf{X}_{8}=y \partial_{t}-\frac{y}{a}\left(x \partial_{x}+z \partial_{z}\right)+\frac{1}{2 a}\left(-a^{2} e^{-2 t / a}+x^{2}-y^{2}+z^{2}\right) \partial_{y}$
$\mathbf{X}_{9}=x \partial_{t}-\frac{x}{a}\left(y \partial_{y}+z \partial_{z}\right)+\frac{1}{2 a}\left(-a^{2} e^{-2 t / a}-x^{2}+y^{2}+z^{2}\right) \partial_{x}$

positive curvature. de Sitter space is an Einstein manifold since the Ricci tensor $R_{i j}$ is proportional to the metric tensor $g_{i j}$. It admits eleven Noether symmetries, out of which seven of them along with their conserved quantities are given in Table 2 and the remaining four correspond to the minimal set given in (7). The structure constants of the associated Lie algebra and the nonzero components of the relevant tensors appear below the equation.

$$
d s^{2}=d t^{2}-e^{2 t / a}\left(d x^{2}+d y^{2}+d z^{2}\right), \quad(a \neq 0) .
$$

$$
\begin{gathered}
C_{i j}^{k} \\
C_{12}^{3}=C_{04}^{3}=C_{05}^{2}=C_{15}^{4}=C_{17}^{8}=C_{45}^{1}=C_{48}^{9}=C_{49}^{8} \\
=C_{57}^{9}=C_{59}^{7}=-1, \\
C_{13}^{2}=C_{09}^{6}=C_{14}^{5}=C_{18}^{7}=C_{25}^{0}=C_{27}^{6}=C_{34}^{0}=C_{38}^{6}=1, \\
C_{06}^{0}=C_{07}^{5}=C_{08}^{4}=C_{26}^{2}=C_{36}^{3}=C_{37}^{1}=C_{67}^{7}=C_{68}^{8} \\
=C_{69}^{6}=-\frac{1}{a}, \\
C_{28}^{1}=C_{29}^{5}=C_{39}^{4}=\frac{1}{a} . \\
R_{i j k l} \\
R_{0101}=R_{0202}=R_{0303}=\frac{1}{a^{2}} e^{2 t / a}, \\
R_{1212}=R_{1313}=R_{2323}=-\frac{1}{a^{2}} e^{4 t / a} . \\
R_{i j} \quad \\
R_{00}=-\frac{3}{a^{2}}, \\
R_{11}=R_{22}=R_{33}=\frac{3}{a^{2}} e^{t / a} .
\end{gathered}
$$

Conserved quantity: $\varphi\left(s, q^{i}, \dot{q}^{i}\right)$

$2 e^{2 t / a} \dot{x}$

$2 e^{2 t / a}(y \dot{x}-x \dot{y})$

$2 e^{2 t / a}(z \dot{x}-x \dot{z})$

$-2\left[\dot{t}+\frac{1}{a} e^{2 t / a}(x \dot{x}+y \dot{y}+z \dot{z})\right]$

$-2\left[z \dot{t}+\frac{z}{a} e^{2 t / a}(x \dot{x}+y \dot{y})-\frac{1}{2 a}\left(-a^{2} e^{-2 t / a}+x^{2}+y^{2}-z^{2}\right) e^{2 t / a} \dot{z}\right]$

$-2\left[y \dot{t}+\frac{y}{a} e^{2 t / a}(x \dot{x}+z \dot{z})-\frac{1}{2 a}\left(-a^{2} e^{-2 t / a}+x^{2}-y^{2}+z^{2}\right) e^{2 t / a} \dot{y}\right]$

$2\left[x \dot{t}-\frac{x}{a} e^{2 t / a}(y \dot{y}+z \dot{z})+\frac{1}{2 a}\left(-a^{2} e^{-2 t / a}-x^{2}+y^{2}+z^{2}\right) e^{2 t / a} \dot{t}\right]$
$R_{i}^{i}$

$$
R=-\frac{12}{a^{2}}
$$

$T_{i j}$

$$
\begin{aligned}
& \kappa T_{00}=\frac{3}{a^{2}}, \\
& \kappa T_{11}=\kappa T_{22}=\kappa T_{33}=-\frac{3}{a^{2}} e^{2 t / a} .
\end{aligned}
$$

$T^{i}{ }_{i}$

$$
T=\frac{12}{a^{2}} .
$$

The Noether symmetries $\mathbf{X}_{0}, \mathbf{X}_{4}$, and $\mathbf{X}_{5}$ correspond to the conservation of linear momentum in $x$-direction and angular momentums, respectively. The conservation laws corresponding to $\mathbf{X}_{6}, \mathbf{X}_{7}, \mathbf{X}_{8}$, and $\mathbf{X}_{9}$ are Killings of the spacetime manifold $M$, associated with different manifestations of generalized momenta. The particular local forms of such geometric symmetries of the underlying space depend upon the chosen coordinate system. For example, if we consider a transformation $\tilde{t}=a e^{-t / a}$ ( $a$ is a constant) then metric (20) takes the following form [38]:

$$
d s^{2}=\left(\frac{a}{\widetilde{t}}\right)^{2}\left(d \tilde{t}^{2}-d x^{2}-d y^{2}-d z^{2}\right), \quad(a \neq 0)
$$

which is an alternative form of the de Sitter metric in conformally flat coordinates. The Noether symmetries in this representation have exactly the same form as given in Table 2 except for $\mathbf{X}_{6}$ to $\mathbf{X}_{9}$ which, in the new coordinates, are given in Table 3. The generators $\mathbf{X}_{7}, \mathbf{X}_{8}$, and $\mathbf{X}_{9}$ in Table 3 can be obtained by conjugation of $\mathbf{X}_{2}, \mathbf{X}_{3}$, and $\mathbf{X}_{0}$, respectively, with the inversion map through a hyperboloid $q^{i}=q^{i} / q^{j} q_{j}$ (in a similar way to what happens for conformal vector fields of the Minkowski metric, cf. [36, Section 9]), since Killings of the 
TABLE 3: Conserved quantities corresponding to each Noether symmetry.

\begin{tabular}{|c|c|}
\hline Generator & Conserved quantity: $\varphi\left(s, q^{i}, \dot{q}^{i}\right)$ \\
\hline $\mathbf{X}_{6}=\widetilde{t} \partial_{\bar{t}}+x \partial_{x}+y \partial_{y}+z \partial_{z}$ & $-2\left(\frac{a}{\tilde{t}}\right)^{2}(\tilde{t} \dot{\vec{t}}-x \dot{x}-y \dot{y}-z \dot{z})$ \\
\hline $\mathbf{X}_{7}=\frac{1}{2}\left(\widetilde{t}^{2}-x^{2}-y^{2}+z^{2}\right) \partial_{z}+z\left(\widetilde{t} \partial_{\tilde{t}}+x \partial_{x}+y \partial_{y}\right)$ & $2\left(\frac{a}{\tilde{t}}\right)^{2}\left[-z(\tilde{t} \dot{\vec{t}}-x \dot{x}-y \dot{y})+\frac{1}{2}\left(\widetilde{t}^{2}-x^{2}-y^{2}+z^{2}\right) \dot{z}\right.$ \\
\hline $\mathbf{X}_{8}=\frac{1}{2}\left(\widetilde{t}^{2}-x^{2}+y^{2}-z^{2}\right) \partial_{y}+y\left(\widetilde{t} \partial_{\tilde{t}}+x \partial_{x}+z \partial_{z}\right)$ & $2\left(\frac{a}{\tilde{t}}\right)^{2}\left[-y(\tilde{t} \dot{\vec{t}}-x \dot{x}-z \dot{z})+\frac{1}{2}\left(\widetilde{t}^{2}-x^{2}+y^{2}-z^{2}\right) \dot{y}\right.$ \\
\hline $\mathbf{X}_{9}=\frac{1}{2}\left(\widetilde{t}^{2}+x^{2}-y^{2}-z^{2}\right) \partial_{x}+x\left(\tilde{t}_{\partial_{t}}+y \partial_{y}+z \partial_{z}\right)$ & $2\left(\frac{a}{\tilde{t}}\right)^{2}\left[-x(\tilde{t} \dot{\tilde{t}}-y \dot{y}-z \dot{z})+\frac{1}{2}\left(\widetilde{t}^{2}+x^{2}-y^{2}-z^{2}\right) \dot{x}\right.$ \\
\hline
\end{tabular}

TABLE 4: Conserved quantities corresponding to each Noether symmetry.

\begin{tabular}{ll}
\hline Generator $\left(\right.$ with $\left.\zeta_{ \pm}=\left(b^{2} / x^{2}\right) \pm\left(y^{2}-z^{2}\right) / K^{2}\right)$ & Conserved quantity: $\varphi\left(s, q^{i}, \dot{q}^{i}\right)$ \\
\hline $\mathbf{X}_{0}=\partial_{t}$ & $-2\left(\frac{x}{a}\right)^{2} \dot{t}$ \\
$\mathbf{X}_{4}=y \partial_{t}+\left(\frac{K}{a}\right)^{2} t \partial_{y}$ & $2\left(\frac{x}{a}\right)^{2}(y \dot{t}-t \dot{y})$ \\
$\mathbf{X}_{5}=z \partial_{t}+\left(\frac{K}{a}\right)^{2} t \partial_{z}$ & $2\left(\frac{x}{a}\right)^{2}(z \dot{t}-t \dot{z})$ \\
$\mathbf{X}_{6}=-t \partial_{t}+x \partial_{x}-y \partial_{y}-z \partial_{z}$ & $2\left[\left(\frac{x}{a}\right)^{2} t \dot{t}+\left(\frac{b}{x}\right)^{2} x \dot{x}-\left(\frac{x}{K}\right)^{2}(y \dot{y}+z \dot{z})\right]$ \\
$\mathbf{X}_{7}=-\left(\frac{a}{K}\right)^{2} z\left(t \partial_{t}-x \partial_{x}+y \partial_{y}\right)-\frac{1}{2}\left(t^{2}-a^{2} \zeta_{+}\right) \partial_{z}$ & $2\left(\frac{x}{K}\right)^{2}\left[z t \dot{t}-z\left(\frac{a}{K}\right)^{2} y \dot{y}+\frac{1}{2}\left(t^{2}-a^{2} \zeta_{+}\right) \dot{z}\right]+2 z\left(\frac{a}{K}\right)^{2}\left(\frac{b}{x}\right)^{2} x \dot{x}$ \\
$\mathbf{X}_{8}=-\left(\frac{a}{K}\right)^{2} y\left(t \partial_{t}-x \partial_{x}+z \partial_{z}\right)-\frac{1}{2}\left(t^{2}-a^{2} \zeta_{-}\right) \partial_{y}$ & $2\left(\frac{x}{K}\right)^{2}\left[y t \dot{t}-y\left(\frac{a}{K}\right)^{2} z \dot{z}+\frac{1}{2}\left(t^{2}-a^{2} \zeta_{-}\right) \dot{y}\right]+2 y\left(\frac{a}{K}\right)^{2}\left(\frac{b}{x}\right)^{2} x \dot{x}$ \\
$\mathbf{X}_{9}=t\left(x \partial_{x}-y \partial_{y}-z \partial_{z}\right)-\frac{1}{2}\left[t^{2}+a^{2}\left(\frac{b^{2}}{x^{2}}+\frac{z^{2}+y^{2}}{K^{2}}\right)\right] \partial_{t}$ & {$\left[t^{2}+a^{2}\left(\frac{b^{2}}{x^{2}}+\frac{z^{2}+y^{2}}{K^{2}}\right)\right]\left(\frac{x}{a}\right)^{2} \dot{t}+2\left[t\left(\frac{b}{x}\right)^{2} x \dot{x}-t\left(\frac{x}{K}\right)^{2}(y \dot{y}+z \dot{z})\right]$} \\
\hline
\end{tabular}

TABLE 5: Conserved quantities corresponding to each Noether symmetry.

\begin{tabular}{ll}
\hline Generator & Conserved Quantity: $\varphi\left(s, q^{i}, \dot{q}^{i}\right)$ \\
\hline $\mathbf{X}_{0}=\partial_{t}$ & $-2 e^{2 x / a} \dot{t}$ \\
$\mathbf{X}_{4}=y \partial_{t}+t \partial_{y}$ & $2 e^{2 x / a}(y \dot{t}-t \dot{y})$ \\
$\mathbf{X}_{5}=z \partial_{t}+t \partial_{z}$ & $2 e^{2 x / a}(z \dot{t}-t \dot{z})$ \\
$\mathbf{X}_{6}=\partial_{x}-\frac{1}{a}\left(t \partial_{t}+y \partial_{y}+z \partial_{z}\right)$ & $2\left[\dot{x}+\frac{1}{a} e^{2 x / a}(t \dot{t}-y \dot{y}-z \dot{z})\right]$ \\
$\mathbf{X}_{7}=z \partial_{x}-\frac{z}{a}\left(t \partial_{t}+y \partial_{y}\right)+\frac{1}{2 a}\left(a^{2} e^{-2 x / a}-t^{2}+y^{2}-z^{2}\right) \partial_{z}$ & $2\left[z \dot{x}+\frac{z}{a} e^{2 x / a}(t \dot{t}-y \dot{y})+\frac{1}{2 a}\left(a^{2} e^{-2 x / a}-t^{2}+y^{2}-z^{2}\right) e^{2 x / a} \dot{z}\right]$ \\
$\mathbf{X}_{8}=y \partial_{x}-\frac{y}{a}\left(t \partial_{t}+z \partial_{z}\right)+\frac{1}{2 a}\left(a^{2} e^{-2 x / a}-t^{2}-y^{2}+z^{2}\right) \partial_{y}$ & $2\left[y \dot{x}+\frac{y}{a} e^{2 x / a}(t \dot{t}-z \dot{z})+\frac{1}{2 a}\left(a^{2} e^{-2 x / a}-t^{2}-y^{2}+z^{2}\right) e^{2 x / a} \dot{y}\right]$ \\
$\mathbf{X}_{9}=t \partial_{x}-\frac{t}{a}\left(y \partial_{y}+z \partial_{z}\right)-\frac{1}{2 a}\left(a^{2} e^{-2 x / a}+t^{2}+y^{2}+z^{2}\right) \partial_{t}$ & $2\left[t \dot{x}-\frac{t}{a} e^{2 x / a}(y \dot{y}+z \dot{z})+\frac{1}{2 a}\left(a^{2} e^{-2 x / a}+t^{2}+y^{2}+z^{2}\right) e^{2 x / a} \dot{t}\right]$ \\
\hline
\end{tabular}

de Sitter metric (27) are conformal Killings of the Minkowski metric (in variables $\tilde{t}, x, y, z$ ).

Anti-de Sitter Space-Times. In this section, the metrics are variants of anti-de Sitter space-times in flat coordinates, maximally symmetric manifold with constant nonzero negative curvature. All the metrics presented here admit the minimal set of Noether symmetries (7) and the remaining symmetry generators along with their conserved quantities (or conservation laws) are given in Tables 4 and 5, respectively. The structure constants of the corresponding Lie algebras and the nonzero components of the relevant tensors are also given after each equation.

Case 1.

$$
\begin{array}{r}
d s^{2}=\left(\frac{x}{a}\right)^{2} d t^{2}-\left(\frac{b}{x}\right)^{2} d x^{2}-\left(\frac{x}{K}\right)^{2}\left(d y^{2}+d z^{2}\right), \\
(a \neq b \neq K \neq 0) .
\end{array}
$$




$$
\begin{aligned}
& C_{i j}^{k} \\
& C_{04}^{3}=C_{05}^{2}=C_{49}^{8}=C_{59}^{7}=\left(\frac{K}{a}\right)^{2} \\
& C_{07}^{5}=C_{08}^{4}=C_{45}^{1}=-\left(\frac{K}{a}\right)^{2}, \\
& C_{27}^{6}=C_{28}^{1}=C_{38}^{6}=\left(\frac{a}{K}\right)^{2}, \\
& C_{29}^{5}=C_{37}^{1}=C_{39}^{4}=-\left(\frac{a}{K}\right)^{2}, \\
& C_{13}^{2}=C_{09}^{6}=C_{14}^{5}=C_{18}^{7}=C_{25}^{0}=C_{34}^{0}=C_{48}^{9}=C_{57}^{9} \\
&=1 \\
& C_{12}^{3}=C_{06}^{0}=C_{15}^{4}=C_{17}^{8}=C_{26}^{2}=C_{36}^{3}=C_{67}^{7}=C_{68}^{8} \\
& \quad=C_{69}^{6}=-1 . \\
& R_{i j k l}
\end{aligned}
$$$$
\begin{aligned}
& R_{0101}=-\frac{1}{a^{2}} \\
& R_{0202}=R_{0303}=-\left(\frac{x^{2}}{a b K}\right)^{2} \\
& R_{1212}=R_{1313}=\frac{1}{K^{2}} \\
& R_{2323}=\left(\frac{x^{2}}{b K}\right)^{2}
\end{aligned}
$$

$R_{i j}$

$$
\begin{aligned}
& R_{00}=3\left(\frac{x}{a b}\right)^{2}, \\
& R_{11}=-\frac{3}{x^{2}} \\
& R_{22}=R_{33}=-3\left(\frac{x}{b K}\right)^{2} .
\end{aligned}
$$

$R_{i}^{i}$

$$
R=\frac{12}{b^{2}}
$$

$T_{i j}$

$$
\begin{aligned}
& \kappa T_{00}=-3\left(\frac{x}{a}\right)^{2}, \\
& \kappa T_{11}=\frac{3}{x^{2}}, \\
& \kappa T_{22}=\kappa T_{33}=3\left(\frac{x}{b K}\right)^{2} .
\end{aligned}
$$

$T_{i}^{i}$

$$
T=-\frac{12}{b^{2}} .
$$

Case 2.

$$
\begin{aligned}
d s^{2} & =e^{2 x / a}\left(d t^{2}-d y^{2}-d z^{2}\right)-d x^{2}, \quad(a \neq 0) . \\
& C_{i j}^{k} \\
C_{13}^{2} & =C_{04}^{3}=C_{05}^{2}=C_{09}^{6}=C_{14}^{5}=C_{18}^{7}=C_{25}^{0}=C_{27}^{6} \\
& =C_{34}^{0}=C_{38}^{6}=C_{48}^{9}=C_{49}^{8}=C_{57}^{9}=C_{59}^{7}=C_{67}^{7} \\
& =C_{68}^{8}=C_{69}^{6}=1, \\
C_{12}^{3} & =C_{15}^{4}=C_{17}^{8}=C_{45}^{1}=-1, \\
C_{28}^{1} & =\frac{1}{a}, \\
C_{06}^{0} & =C_{07}^{5}=C_{08}^{4}=C_{26}^{2}=C_{29}^{5}=C_{36}^{3}=C_{37}^{1}=C_{39}^{4} \\
& =-\frac{1}{a} . \\
& R_{i j k l}
\end{aligned}
$$

$$
\begin{aligned}
& R_{00}=\frac{3}{a^{2}} e^{2 x / a}, \\
& R_{11}=-\frac{3}{a^{2}}, \\
& R_{22}=R_{33}=-\frac{3}{a^{2}} e^{2 x / a} .
\end{aligned}
$$

$R_{i}^{i}$

$$
R=\frac{12}{a^{2}} .
$$

$T_{i j}$

$$
\begin{aligned}
\kappa T_{00} & =-\frac{3}{a^{2}} e^{2 x / a}, \\
\kappa T_{11} & =\frac{3}{a^{2}}, \\
T_{22} & =\kappa T_{33}=\frac{3}{a^{2}} e^{2 x / a} .
\end{aligned}
$$


TABLE 6: Conserved quantities corresponding to each Noether symmetry.

\begin{tabular}{ll}
\hline $\begin{array}{l}\text { Generator; gauge term: } \\
A\left(s, q^{i}\right)\end{array}$ & Conserved quantity: $\varphi\left(s, q^{i}, \dot{q}^{i}\right)$ \\
\hline $\mathbf{X}_{0}=\partial_{t}$ & $-2 e^{2 x / a} \dot{t}$ \\
$\mathbf{X}_{4}=$ & $\frac{1}{a}\left(t^{2}+a^{2} e^{-2 x / a}\right) e^{2 x / a} \dot{t}+2 t \dot{x}$ \\
$-\frac{1}{2 a}\left(t^{2}+a^{2} e^{-2 x / a}\right) \partial_{t}+t \partial_{x}$ & $2\left(\frac{1}{a} e^{2 x / a} t \dot{t}+\dot{x}\right)$ \\
$\mathbf{X}_{5}=-\frac{1}{a} t \partial_{t}+\partial_{x}$ & $2(y-s \dot{y})$ \\
$\mathbf{Y}_{1}=s \partial_{y} ; A_{1}=-2 y$ & $2(z-s \dot{z})$ \\
$\mathbf{Y}_{2}=s \partial_{z} ; A_{2}=-2 z$ &
\end{tabular}

TABLE 7: Conserved quantities corresponding to each Noether symmetry.

\begin{tabular}{ll}
\hline $\begin{array}{l}\text { Generator; gauge } \\
\text { term: } A\left(s, q^{i}\right)\end{array}$ & Conserved quantity: $\varphi\left(s, q^{i}, \dot{q}^{i}\right)$ \\
\hline $\mathbf{X}_{0}=\partial_{x}$ & $2 e^{2 t / a} \dot{x}$ \\
$\mathbf{X}_{4}=x \partial_{t}-$ & $-2\left(x \dot{t}+\frac{1}{2 a}\left(a^{2} e^{-2 t / a}+x^{2}\right) e^{2 t / a} \dot{x}\right)$ \\
$\frac{1}{2 a}\left(a^{2} e^{-2 t / a}+x^{2}\right) \partial_{x}$ & $-2\left[\dot{t}+\frac{1}{a} e^{2 t / a} x \dot{x}\right]$ \\
$\mathbf{X}_{5}=\partial_{t}-\frac{x}{a} \partial_{x}$ & $2(y-s \dot{y})$ \\
$\mathbf{Y}_{1}=s \partial_{y} ; A_{1}=-2 y$ & $2(z-s \dot{z})$ \\
$\mathbf{Y}_{2}=s \partial_{z} ; A_{2}=-2 z$ & \\
\hline
\end{tabular}

$$
T_{i}^{i}
$$

$$
T=-\frac{12}{a^{2}}
$$

The conservation laws corresponding to the Noether symmetries $\mathbf{X}_{6}-\mathbf{X}_{9}$ in this case are similar to the ones of de Sitter space-time.

4.3. Nine Noether Symmetries. In this section, all metrics admit nine Noether symmetries out of which four (minimal set) are given in (7) and the remaining symmetry generators along with their conserved quantities are presented in Tables 6-11, respectively. The structure constants and the nonzero components of the relevant tensors are also calculated in each case. Some of the metrics are variants of well-known spacetimes that are solutions of the EFEs.

Bertotti-Robinson Space-Times. The following are the list of Bertotti-Robinson-like metrics.

Case 1.

$$
\begin{aligned}
& d s^{2}=e^{2 x / a} d t^{2}-d x^{2}-d y^{2}-d z^{2}, \quad(a \neq 0) . \\
& C_{i j}^{k} \\
& \\
& \quad C_{13}^{2}=C_{\widehat{01}}^{3}=C_{\widehat{02}}^{2}=C_{1 \hat{1}}^{\hat{2}}=C_{04}^{5}=1, \\
& C_{12}^{3}=C_{1 \hat{2}}^{\widehat{1}}=-1,
\end{aligned}
$$

$$
\begin{aligned}
& C_{05}^{0}=-\frac{1}{a}, \\
& C_{45}^{4}=\frac{1}{a} .
\end{aligned}
$$

$R_{i j k l}$

$$
R_{0101}=-\frac{1}{a^{2}} e^{2 x / a}
$$

$R_{i j}$

$$
\begin{aligned}
& R_{00}=-\frac{1}{a^{2}} e^{2 x / a}, \\
& R_{11}=-\frac{1}{a^{2}}
\end{aligned}
$$

$R_{i}^{i}$

$$
R=\frac{2}{a^{2}}
$$

$T_{i j}$

$$
\kappa T_{22}=\kappa T_{33}=\frac{1}{a^{2}}
$$

$T_{i}^{i}$

$$
T=-\frac{2}{a^{2}}
$$

Case 2.

$$
d s^{2}=d t^{2}-e^{2 t / a} d x^{2}-d y^{2}-d z^{2}, \quad(a \neq 0) .
$$

The structure constants of the corresponding Lie algebra are the same as for metric (42).

$$
\begin{aligned}
R_{i j k l} & R_{0101}=\frac{1}{a^{2}} e^{2 t / a} . \\
R_{i j} & R_{00}=-\frac{1}{a^{2}}, \\
R_{11} & =\frac{1}{a^{2}} e^{2 t / a} . \\
R_{i}^{i} \quad R & =-\frac{2}{a^{2}} . \\
T_{i j} \quad & \kappa T_{22}=\kappa T_{33}=-\frac{1}{a^{2}} .
\end{aligned}
$$


TABLE 8: Conserved quantities corresponding to each Noether symmetry.

\begin{tabular}{ll}
\hline Generator; gauge term: $A\left(s, q^{i}\right)$ & Conserved quantity: $\varphi\left(s, q^{i}, \dot{q}^{i}\right)$ \\
\hline $\mathbf{X}_{0}=\partial_{t}$ & $-2 \cos ^{2}\left(\frac{x}{a}\right) \dot{t}$ \\
$\mathbf{X}_{4}=\tan \left(\frac{x}{a}\right) \sinh \left(\frac{t}{a}\right) \partial_{t}+\cosh \left(\frac{t}{a}\right) \partial_{x}$ & $-2 \sin \left(\frac{x}{a}\right) \cos \left(\frac{x}{a}\right) \sinh \left(\frac{t}{a}\right) \dot{t}+2 \cosh \left(\frac{t}{a}\right) \dot{x}$ \\
$\mathbf{X}_{5}=\tan \left(\frac{x}{a}\right) \cosh \left(\frac{t}{a}\right) \partial_{t}+\sinh \left(\frac{t}{a}\right) \partial_{x}$ & $-2 \sin \left(\frac{x}{a}\right) \cos \left(\frac{x}{a}\right) \cosh \left(\frac{t}{a}\right) \dot{t}+2 \sinh \left(\frac{t}{a}\right) \dot{x}$ \\
$\mathbf{Y}_{1}=s \partial_{y} ; A_{1}=-2 y$ & $2(y-s \dot{y})$ \\
$\mathbf{Y}_{2}=s \partial_{z} ; A_{2}=-2 z$ & $2(z-s \dot{z})$ \\
\hline
\end{tabular}

TABLE 9: Conserved quantities corresponding to each Noether symmetry.

\begin{tabular}{ll}
\hline Generator; gauge term: $A\left(s, q^{i}\right)$ & Conserved quantity: $\varphi\left(s, q^{i}, \dot{q}^{i}\right)$ \\
\hline $\mathbf{X}_{0}=\partial_{x}$ & $2 \cos ^{2}\left(\frac{t}{a}\right) \dot{x}$ \\
$\mathbf{X}_{4}=\cosh \left(\frac{x}{a}\right) \partial_{t}+\tan \left(\frac{t}{a}\right) \sinh \left(\frac{x}{a}\right) \partial_{x}$ & $-2 \cosh \left(\frac{x}{a}\right) \dot{t}+2 \sin \left(\frac{t}{a}\right) \cos \left(\frac{t}{a}\right) \sinh \left(\frac{x}{a}\right) \dot{x}$ \\
$\mathbf{X}_{5}=\sinh \left(\frac{x}{a}\right) \partial_{t}+\tan \left(\frac{t}{a}\right) \cosh \left(\frac{x}{a}\right) \partial_{x}$ & $-2 \sinh \left(\frac{x}{a}\right) \dot{t}+2 \sin \left(\frac{t}{a}\right) \cos \left(\frac{t}{a}\right) \cosh \left(\frac{x}{a}\right) \dot{x}$ \\
$\mathbf{Y}_{1}=s \partial_{y} ; A_{1}=-2 y$ & $2(y-s \dot{y})$ \\
$\mathbf{Y}_{2}=s \partial_{z} ; A_{2}=-2 z$ & $2(y-s \dot{y})$ \\
\hline
\end{tabular}

TABLE 10: Conserved quantities corresponding to each Noether symmetry.

\begin{tabular}{ll}
\hline Generator; gauge term: $A\left(s, q^{i}\right)$ & Conserved quantity: $\varphi\left(s, q^{i}, \dot{q}^{i}\right)$ \\
\hline $\mathbf{X}_{0}=\partial_{t}$ & $-2 \cosh ^{2}\left(\frac{x}{a}\right) \dot{t}$ \\
$\mathbf{X}_{4}=-\tanh \left(\frac{x}{a}\right) \sin \left(\frac{t}{a}\right) \partial_{t}+\cos \left(\frac{t}{a}\right) \partial_{x}$ & $2 \sinh \left(\frac{x}{a}\right) \cosh \left(\frac{x}{a}\right) \sin \left(\frac{t}{a}\right) \dot{t}+2 \cos \left(\frac{t}{a}\right) \dot{x}$ \\
$\mathbf{X}_{5}=\tanh \left(\frac{x}{a}\right) \cos \left(\frac{t}{a}\right) \partial_{t}+\sin \left(\frac{t}{a}\right) \partial_{x}$ & $-2 \sinh \left(\frac{x}{a}\right) \cosh \left(\frac{x}{a}\right) \cos \left(\frac{t}{a}\right) \dot{t}+2 \sin \left(\frac{t}{a}\right) \dot{x}$ \\
$\mathbf{Y}_{1}=s \partial_{y} ; A_{1}=-2 y$ & $2(y-s \dot{y})$ \\
$\mathbf{Y}_{2}=s \partial_{z} ; A_{2}=-2 z$ & $2(z-s \dot{z})$ \\
\hline
\end{tabular}

TABLE 11: Conserved quantities corresponding to each Noether symmetry.

\begin{tabular}{ll}
\hline Generator; gauge term: $A\left(s, q^{i}\right)$ & Conserved quantity: $\varphi\left(s, q^{i}, \dot{q}^{i}\right)$ \\
\hline $\mathbf{X}_{0}=\partial_{x}$ & $2 \cosh ^{2}\left(\frac{t}{a}\right) \dot{x}$ \\
$\mathbf{X}_{4}=\cos \left(\frac{x}{a}\right) \partial_{t}-\tanh \left(\frac{t}{a}\right) \sin \left(\frac{x}{a}\right) \partial_{x}$ & $-2 \cos \left(\frac{x}{a}\right) \dot{t}-2 \sinh \left(\frac{t}{a}\right) \cosh \left(\frac{t}{a}\right) \sin \left(\frac{x}{a}\right) \dot{x}$ \\
$\mathbf{X}_{5}=\sin \left(\frac{x}{a}\right) \partial_{t}+\tanh \left(\frac{t}{a}\right) \cos \left(\frac{x}{a}\right) \partial_{x}$ & $-2 \sin \left(\frac{x}{a}\right) \dot{t}+2 \sinh \left(\frac{t}{a}\right) \cosh \left(\frac{t}{a}\right) \cos \left(\frac{x}{a}\right) \dot{x}$ \\
$\mathbf{Y}_{1}=s \partial_{y} ; A_{1}=-2 y$ & $2(y-s \dot{y})$ \\
$\mathbf{Y}_{2}=s \partial_{z} ; A_{2}=-2 z$ & $2(z-s \dot{z})$ \\
\hline
\end{tabular}

$T_{i}^{i}$

$$
T=\frac{2}{a^{2}} .
$$

Case 3.

$$
d s^{2}=\cos ^{2}\left(\frac{x}{a}\right) d t^{2}-d x^{2}-d y^{2}-d z^{2}, \quad(a \neq 0) .
$$

$C_{i j}^{k}$

$$
C_{04}^{5}=C_{05}^{4}=C_{45}^{0}=\frac{1}{a},
$$

$C_{12}^{3}=C_{1 \widehat{2}}^{\widehat{1}}=-1$,

$C_{13}^{2}=C_{\widehat{01}}^{3}=C_{\widehat{02}}^{2}=C_{1 \widehat{1}}^{\hat{2}}=1$. 
$R_{i j k l}$

$$
R_{0101}=\frac{1}{a^{2}} \cos ^{2}\left(\frac{x}{a}\right)
$$

$R_{i j}$

$$
\begin{aligned}
& R_{00}=-\frac{1}{a^{2}} \cos ^{2}\left(\frac{x}{a}\right), \\
& R_{11}=\frac{1}{a^{2}} .
\end{aligned}
$$

$R_{i}^{i}$

$$
R=-\frac{2}{a^{2}} .
$$

$T_{i j}$

$$
\kappa T_{22}=\kappa T_{33}=-\frac{1}{a^{2}} .
$$

$T_{i}^{i}$

$$
T=\frac{2}{a^{2}} .
$$

Case 4.

$$
d s^{2}=d t^{2}-\cos ^{2}\left(\frac{t}{a}\right) d x^{2}-d y^{2}-d z^{2}, \quad(a \neq 0) .
$$

The structure constants of the corresponding Lie algebra are the same as for (55).

$R_{i j k l}$

$$
R_{0101}=-\frac{1}{a^{2}} \cos ^{2}\left(\frac{t}{a}\right)
$$

$R_{i j}$

$$
\begin{aligned}
& R_{00}=\frac{1}{a^{2}}, \\
& R_{11}=-\frac{1}{a^{2}} \cos ^{2}\left(\frac{t}{a}\right) .
\end{aligned}
$$

$R_{i}^{i}$

$$
R=\frac{2}{a^{2}} .
$$

$T_{i j}$

$$
\kappa T_{22}=\kappa T_{33}=\frac{1}{a^{2}} .
$$

$T_{i}^{i}$

$$
T=-\frac{2}{a^{2}} .
$$

Case 5.

$$
d s^{2}=\cosh ^{2}\left(\frac{x}{a}\right) d t^{2}-d x^{2}-d y^{2}-d z^{2}, \quad(a \neq 0) .
$$

$$
C_{i j}^{k}
$$

$$
\begin{aligned}
& C_{13}^{2}=C_{\widehat{0} \hat{1}}^{3}=C_{\widehat{0} \hat{2}}^{2}=C_{1 \widehat{1}}^{\hat{2}}=1, \\
& C_{04}^{5}=-\frac{1}{a}, \\
& C_{05}^{4}=C_{45}^{0}=\frac{1}{a}, \\
& C_{12}^{3}=C_{1 \widehat{1}}^{\widehat{1}}=-1 .
\end{aligned}
$$

$R_{i j k l}$

$$
R_{0101}=-\frac{1}{a^{2}} \cosh ^{2}\left(\frac{x}{a}\right) .
$$

$R_{i j}$

$$
\begin{aligned}
& R_{00}=\frac{1}{a^{2}} \cosh ^{2}\left(\frac{x}{a}\right), \\
& R_{11}=-\frac{1}{a^{2}} .
\end{aligned}
$$

$R_{i}^{i}$

$$
R=\frac{2}{a^{2}} \text {. }
$$

$T_{i j}$

$$
\kappa T_{22}=\kappa T_{33}=\frac{1}{a^{2}} .
$$

$T^{i}{ }_{i}$

$$
T=-\frac{2}{a^{2}} .
$$

Case 6.

$$
d s^{2}=d t^{2}-\cosh ^{2}\left(\frac{t}{a}\right) d x^{2}-d y^{2}-d z^{2}, \quad(a \neq 0) .
$$

The structure constants of the corresponding Lie algebra are the same as for (68).

$$
R_{i j k l}
$$

$$
R_{0101}=\frac{1}{a^{2}} \cosh ^{2}\left(\frac{t}{a}\right) .
$$

$R_{i j}$

$$
\begin{aligned}
& R_{00}=-\frac{1}{a^{2}}, \\
& R_{11}=\frac{1}{a^{2}} \cosh ^{2}\left(\frac{t}{a}\right) .
\end{aligned}
$$


TABLE 12: Conserved quantities corresponding to each Noether symmetry.

\begin{tabular}{ll}
\hline Generator; gauge term: $A\left(s, q^{i}\right)$ & Conserved quantity: $\varphi\left(s, q^{i}, \dot{q}^{i}\right)$ \\
\hline $\mathbf{X}_{0}=\partial_{x}$ & $2 \dot{x}$ \\
$\mathbf{X}_{4}=\partial_{t}-\frac{1}{a}\left(y \partial_{y}+z \partial_{z}\right)$ & $2\left[\dot{t}-\frac{1}{a} e^{2 t / a}(y \dot{y}+z \dot{z})\right]$ \\
$\mathbf{X}_{5}=y \partial_{t}+\frac{1}{2 a}\left(-a^{2} e^{-2 t / a}-y^{2}+z^{2}\right) \partial_{y}-\frac{y z}{a} \partial_{z}$ & $2\left[-y \dot{t}+e^{2 t / a}\left\{\frac{1}{2 a}\left(-a^{2} e^{-2 t / a}-y^{2}+z^{2}\right) \dot{y}-\frac{y}{a} z \dot{z}\right\}\right]$ \\
$\mathbf{X}_{6}=z \partial_{t}+\frac{1}{2 a}\left(-a^{2} e^{-2 t / a}+y^{2}-z^{2}\right) \partial_{z}-\frac{y z}{a} \partial_{y}$ & $2\left[-z \dot{t}+e^{2 t / a}\left\{\frac{1}{2 a}\left(-a^{2} e^{-2 t / a}-y^{2}+z^{2}\right) \dot{z}-\frac{z}{a} y \dot{y}\right\}\right]$ \\
$\mathbf{Y}_{1}=s \partial_{x} ; A_{1}=-2 x$ & $2(x-s \dot{x})$ \\
\hline
\end{tabular}

$R_{i}^{i}$

$$
R=-\frac{2}{a^{2}}
$$

$T_{i j}$

$$
\kappa T_{22}=\kappa T_{33}=-\frac{1}{a^{2}} .
$$

$T_{i}^{i}$

$$
T=\frac{2}{a^{2}} .
$$

The Noether symmetries $\mathbf{X}_{4}$ and $\mathbf{X}_{5}$ are also geometric collineations of the underlying space that correspond to other KVFs. The conservation law associated with these symmetries is the conservation of a particular form of generalized momentum. As explained in Section 3, there are adapted coordinate frames where these symmetries take the form $f(u, v) \partial_{u}$, along some particular direction $u$ and for an appropriate conformal factor $f(u, v)$. In such a coordinate system, each symmetry is associated with the conservation of momentum along the $u$-direction. The symmetry generators $\mathbf{Y}_{1}$ and $\mathbf{Y}_{2}$ are the Galilean symmetries that can be interpreted geometrically as Killings of the extended space $\mathbb{R} \times M$ of variables $(s, t, x, y, z)$. They represent a change of reference frame to one moving along some geodesic (center of mass frame) in a particular direction.

Einstein Universe. The following metric admits nine Noether symmetries, out of which five along with their conserved forms are given in Table 12 and the remaining four corresponds to the minimal set given in (7). The structure constants of the associated Lie algebra and the nonzero components of the relevant tensors are also calculated.

$$
\begin{aligned}
& d s^{2}=d t^{2}-d x^{2}-e^{2 t / a}\left(d y^{2}+d z^{2}\right), \quad(a \neq 0) . \\
& C_{i j}^{k} \\
& C_{13}^{2}=C_{\widehat{01}}^{0}=C_{15}^{4}=C_{26}^{4}=C_{35}^{1}=C_{46}^{6}=1, \\
& C_{12}^{3}=C_{16}^{5}=-1,
\end{aligned}
$$

$$
\begin{aligned}
& C_{24}^{2}=C_{34}^{3}=C_{45}^{5}=C_{36}^{1}=-\frac{1}{a}, \\
& C_{25}^{1}=\frac{1}{a} .
\end{aligned}
$$

$R_{i j k l}$

$$
\begin{aligned}
& R_{0202}=R_{0303}=\frac{1}{a^{2}} e^{2 t / a}, \\
& R_{2323}=-\frac{1}{a^{2}} e^{4 t / a} .
\end{aligned}
$$

$R_{i j}$

$$
\begin{aligned}
& R_{00}=-\frac{2}{a^{2}}, \\
& R_{22}=R_{33}=\frac{2}{a^{2}} e^{2 t / a} .
\end{aligned}
$$

$R_{i}^{i}$

$$
R=-\frac{6}{a^{2}}
$$

$T_{i j}$

$$
\begin{aligned}
& \kappa T_{00}=\frac{1}{a^{2}}, \\
& \kappa T_{11}=-\frac{3}{a^{2}}, \\
& \kappa T_{22}=\kappa T_{33}=-\frac{1}{a^{2}} e^{2 t / a} .
\end{aligned}
$$

$T_{i}^{i}$

$$
T=\frac{6}{a^{2}}
$$

Since de Sitter space-time is conformally related to an Einstein universe, the interpretation of the symmetry generators $\mathbf{X}_{5}$ and $\mathbf{X}_{6}$ is similar to one presented earlier (see Section 4.2). Finally, the Noether symmetry generator $\mathbf{Y}_{1}$ is a Galilean symmetry. This generator, as it was previously 
TABLE 13: Conserved quantities corresponding to each Noether symmetry.

Generator; gauge term: $A\left(s, q^{i}\right)$

$\mathbf{X}_{0}=\partial_{t}$

$\mathbf{X}_{4}=\partial_{x}-\frac{1}{a}\left(y \partial_{y}+z \partial_{z}\right)$

$\mathbf{X}_{5}=y \partial_{x}+\frac{1}{2 a}\left(a^{2} e^{-2 x / a}+z^{2}-y^{2}\right) \partial_{y}-\frac{y z}{a} \partial_{z}$

$\mathbf{X}_{6}=z \partial_{x}-\frac{y z}{a} \partial_{y}+\frac{1}{2 a}\left(a^{2} e^{-2 x / a}+y^{2}-z^{2}\right) \partial_{z}$

$\mathbf{Y}_{1}=s \partial_{t} ; A_{1}=2 t$

Conserved quantity: $\varphi\left(s, q^{i}, \dot{q}^{i}\right)$
$2 \dot{t}$
$2 \dot{x}-\frac{2}{a} e^{2 x / a}(y \dot{y}+z \dot{z})$
$2\left[y \dot{x}+e^{2 x / a}\left\{\frac{1}{2 a}\left(a^{2} e^{-2 x / a}+z^{2}-y^{2}\right) \dot{y}-\frac{y}{a} z \dot{z}\right\}\right]$
$2\left[z \dot{x}+e^{2 x / a}\left\{\frac{1}{2 a}\left(a^{2} e^{-2 x / a}-z^{2}+y^{2}\right) \dot{z}-\frac{z}{a} y \dot{y}\right\}\right]$
$2(t-s \dot{t})$

TABLE 14: Conserved quantities corresponding to each Noether symmetry.

\begin{tabular}{ll}
\hline Generator; gauge term: $A\left(s, q^{i}\right)$ & Conserved quantity: $\varphi\left(s, q^{i}, \dot{q}^{i}\right)$ \\
\hline $\mathbf{X}_{0}=\partial_{t}$ & $-2 \dot{t}$ \\
$\mathbf{X}_{4}=x \partial_{x}-y \partial_{y}-z \partial_{z}$ & $2\left[\left(\frac{a}{x}\right)^{2} x \dot{x}-\left(\frac{x}{K}\right)^{2}(y \dot{y}+z \dot{z})\right]$ \\
$\mathbf{X}_{5}=z x \partial_{x}-z y \partial_{y}+\frac{1}{2}\left(\frac{a^{2} K^{2}}{x^{2}}+y^{2}-z^{2}\right) \partial_{z}$ & $2\left[\left(\frac{a}{x}\right)^{2} z x \dot{x}-\left(\frac{x}{K}\right)^{2}\left\{z y \dot{y}-\frac{1}{2}\left(\frac{a^{2} K^{2}}{x^{2}}+y^{2}-z^{2}\right) \dot{z}\right\}\right]$ \\
$\mathbf{X}_{6}=y x \partial_{x}+\frac{1}{2}\left(\frac{a^{2} K^{2}}{x^{2}}-y^{2}+z^{2}\right) \partial_{y}-y z \partial_{z}$ & $2\left[\left(\frac{a}{x}\right)^{2} y x \dot{x}+\left(\frac{x}{K}\right)^{2}\left\{\frac{1}{2}\left(\frac{a^{2} K^{2}}{x^{2}}-y^{2}+z^{2}\right) \dot{y}-y z \dot{z}\right\}\right]$ \\
$\mathbf{Y}_{1}=s \partial_{t} ; A_{1}=2 t$ & $2(t-s \dot{t})$
\end{tabular}

discussed, corresponds geometrically to a Killing of the parameter space $\mathbb{R} \times M$ represented locally by the coordinates $(s, t, x, y, z)$.

Anti-Einstein Space-Times. These metrics admit nine Noether symmetries (corresponding to their conserved forms) that are given in Tables 13 and 14 (respectively) along with the minimal set (7). Moreover, their structure constants of the associated Lie algebra and the nonzero components of the relevant tensors are also determined.

Case 1.

$$
d s^{2}=d t^{2}-d x^{2}-e^{2 x / a}\left(d y^{2}+d z^{2}\right), \quad(a \neq 0) .
$$

The structure constants of the corresponding Lie algebra are the same as for (81).

$$
\begin{aligned}
R_{i j k l} & R_{1212}=R_{1313}=\frac{1}{a^{2}} e^{2 x / a}, \\
R_{2323} & =\frac{1}{a^{2}} e^{4 x / a} . \\
R_{i j} & R_{11}=-\frac{2}{a^{2}}, \\
R_{22} & =R_{33}=\frac{2}{a^{2}} e^{2 x / a} .
\end{aligned}
$$

$R_{i}^{i}$

$$
R=\frac{6}{a^{2}} .
$$

$T_{i j}$

$$
\begin{aligned}
& \kappa T_{00}=-\frac{3}{a^{2}}, \\
& \kappa T_{11}=\frac{3}{a^{2}}, \\
& \kappa T_{22}=\kappa T_{33}=\frac{1}{a^{2}} e^{2 x / a} .
\end{aligned}
$$

$T^{i}{ }_{i}$

$$
T=-\frac{6}{a^{2}} .
$$

Case 2.

$$
d s^{2}=d t^{2}-\left(\frac{a}{x}\right)^{2} d x^{2}-\left(\frac{x}{K}\right)^{2}\left(d y^{2}+d z^{2}\right),
$$

$(a \neq K \neq 0)$

$$
\begin{gathered}
C_{i j}^{k} \\
C_{13}^{2}=C_{16}^{5}=C_{25}^{4}=C_{26}^{1}=C_{36}^{4}=C_{\widehat{01}}^{0}=1, \\
C_{12}^{3}=C_{15}^{6}=C_{24}^{2}=C_{34}^{3}=C_{35}^{1}=C_{45}^{5}=C_{46}^{6}=-1 .
\end{gathered}
$$

$R_{i j k l}$

$$
R_{2323}=\frac{x^{2}}{K^{4}} .
$$


TABLE 15: Conserved quantities corresponding to each Noether symmetry.

\begin{tabular}{ll}
\hline $\begin{array}{l}\text { Generator; gauge term: } \\
A\left(s, q^{i}\right)\end{array}$ & Conserved quantity: $\varphi\left(s, q^{i}, \dot{q}^{i}\right)$ \\
\hline $\mathbf{X}_{0}=\partial_{t}$ & $-2\left(\frac{x}{a}\right)^{2} \dot{t}$ \\
$\mathbf{X}_{4}=y \partial_{t}+\left(\frac{b}{a}\right)^{2} t \partial_{y}$ & $2\left(\frac{x}{a}\right)^{2}(y \dot{t}-t \dot{y})$ \\
$\mathbf{X}_{5}=z \partial_{t}+\left(\frac{b}{a}\right)^{2} t \partial_{z}$ & $2\left(\frac{x}{a}\right)^{2}(z \dot{t}-t \dot{z})$ \\
$\mathbf{Y}_{1}=\frac{1}{2}\left(s^{2} \partial_{s}+s x \partial_{x}\right) ;$ & $\frac{1}{2} s^{2} L+s x \dot{x}-\frac{1}{2} x^{2}$ \\
$A_{1}=-\frac{1}{2} x^{2}$ & $s L+x \dot{x}$ \\
$\mathbf{Y}_{2}=s \partial_{s}+\frac{x}{2} \partial_{x}$ &
\end{tabular}

$R_{i j}$

$$
R_{22}=R_{33}=-\frac{2}{a^{2}}\left(\frac{x}{K}\right)^{2} \text {. }
$$

$R_{i}^{i}$

$$
R=\frac{6}{a^{2}}
$$

$T_{i j}$

$$
\begin{aligned}
& \kappa T_{00}=-\frac{3}{a^{2}}, \\
& \kappa T_{11}=\frac{1}{x^{2}}, \\
& \kappa T_{22}=\kappa T_{33}=\frac{1}{a^{2}}\left(\frac{x}{K}\right)^{2} .
\end{aligned}
$$

$T_{i}^{i}$

$$
T=-\frac{6}{a^{2}}
$$

The interpretation of these Noether symmetries is similar to the one given for the symmetries of the Einstein universe.

Other Metrics (9 Symmetries). These are the metrics whose corresponding Lagrangian admit nine Noether symmetries, out of which four are given in (7). The symmetry generators along with their conserved quantities are given in Tables 15-20, respectively. Additionally, the structure constants of the associated Lie algebra and the nonzero components of the relevant tensors are explicitly given below for each case. The following list of metrics do not belong to known class of the space-times.
TABLE 16: Conserved quantities corresponding to each Noether symmetry.

\begin{tabular}{ll}
\hline $\begin{array}{l}\text { Generator; gauge term: } \\
A\left(s, q^{i}\right)\end{array}$ & Conserved quantity: $\varphi\left(s, q^{i}, \dot{q}^{i}\right)$ \\
\hline $\mathbf{X}_{0}=\partial_{t}$ & $-2\left(\frac{x}{a}\right)^{2} \dot{t}$ \\
$\mathbf{X}_{4}=y \partial_{t}+t \partial_{y}$ & $2\left(\frac{x}{a}\right)^{2}(y \dot{t}-t \dot{y})$ \\
$\mathbf{X}_{5}=z \partial_{t}+t \partial_{z}$ & $2\left(\frac{x}{a}\right)^{2}(z \dot{t}-t \dot{z})$ \\
$\mathbf{Y}_{1}=\frac{1}{2}\left(s^{2} \partial_{s}+s x \partial_{x}\right) ;$ & $\frac{1}{2} s^{2} L+s x \dot{x}-\frac{1}{2} x^{2}$ \\
$A_{1}=-\frac{1}{2} x^{2}$ & $s L+x \dot{x}$ \\
$\mathbf{Y}_{2}=s \partial_{s}+\frac{x}{2} \partial_{x}$ & \\
\hline
\end{tabular}

TABLE 17: Conserved quantities corresponding to each Noether symmetry.

\begin{tabular}{ll}
\hline $\begin{array}{l}\text { Generator; gauge term: } \\
A\left(s, q^{i}\right)\end{array}$ & Conserved quantity: $\varphi\left(s, q^{i}, \dot{q}^{i}\right)$ \\
\hline $\mathbf{X}_{0}=\partial_{x}$ & $2\left(\frac{t}{a}\right)^{2} \dot{x}$ \\
$\mathbf{X}_{4}=y \partial_{x}-x \partial_{y}$ & $2\left(\frac{t}{a}\right)^{2}(y \dot{x}-x \dot{y})$ \\
$\mathbf{X}_{5}=z \partial_{x}-x \partial_{z}$ & $2\left(\frac{t}{a}\right)^{2}(z \dot{x}-x \dot{z})$ \\
$\mathbf{Y}_{1}=\frac{1}{2}\left(s^{2} \partial_{s}+s t \partial_{t}\right)$, & $\frac{1}{2} s^{2} L-s t \dot{t}+\frac{1}{2} t^{2}$ \\
$A_{1}=\frac{1}{2} t^{2}$ & $s L-t \dot{t}$ \\
$\mathbf{Y}_{2}=s \partial_{s}+\frac{t}{2} \partial_{t}$ &
\end{tabular}

Case 1.

$$
d s^{2}=\left(\frac{x}{a}\right)^{2} d t^{2}-d x^{2}-\left(\frac{x}{b}\right)^{2}\left(d y^{2}+d z^{2}\right),
$$

$(a \neq b \neq 0)$.

$$
\begin{aligned}
C_{i j}^{k} & \\
C_{13}^{2} & =C_{14}^{5}=C_{25}^{0}=C_{34}^{0}=C_{\widehat{0} \hat{1}}^{\widehat{1}}=C_{\widehat{0} \hat{2}}^{\widehat{0}}=1, \\
C_{12}^{3} & =C_{15}^{4}=C_{\widehat{12}}^{\hat{1}}=-1, \\
C_{04}^{3} & =C_{05}^{2}=\left(\frac{b}{a}\right)^{2}, \\
C_{45}^{1} & =-\left(\frac{b}{a}\right)^{2} \cdot \\
R_{i j k l} & \\
R_{0202} & =R_{0303}=-\frac{1}{b^{2}}\left(\frac{x}{a}\right)^{2}, \\
& R_{2323}=\frac{1}{b^{2}}\left(\frac{x}{b}\right)^{2} \cdot
\end{aligned}
$$


TABLE 18: Conserved quantities corresponding to each Noether symmetry.

Generator; gauge term: $A\left(s, q^{i}\right)$

$\mathbf{X}_{0}=-\partial_{t}+\partial_{x}+y \partial_{y}+z \partial_{z}$

$\mathbf{X}_{4}=-z \partial_{t}+z \partial_{x}+y z \partial_{y}+\frac{1}{2}\left(z^{2}-y^{2}-\zeta\right) \partial_{z}$

$\mathbf{X}_{5}=y \partial_{t}-y \partial_{x}+\frac{1}{2}\left(z^{2}-y^{2}+\zeta\right) \partial_{y}-y z \partial_{z}$

$\mathbf{Y}_{1}=\frac{s^{2}}{2}+\frac{s}{2}\left[C_{1}(t, x) \partial_{t}-C_{2}(t, x) \partial_{x}\right] ;$

$A_{1}=\int e^{2 v} C_{1}(t, x) d t+\int e^{2 \lambda} C_{2}(t, x) d x$

$\mathbf{Y}_{2}=s \partial_{s}+\frac{1}{2}\left[C_{1}(t, x) \partial_{t}-C_{2}(t, x) \partial_{x}\right]$

\section{Conserved quantity: $\varphi\left(s, q^{i}, \dot{q}^{i}\right)$}

$2\left[\dot{t} e^{2 \gamma}+\dot{x} e^{2 \lambda}+(y \dot{y}+z \dot{z}) e^{2(t+f(t+x))}\right]$

$2\left[z\left(\dot{t} e^{2 \gamma}+\dot{x} e^{2 \lambda}\right)+e^{2(t+f(t+x))}\left\{y \dot{y} z-\frac{\dot{z}}{2}\left(z^{2}-y^{2}-\zeta\right)\right\}\right]$

$2\left[-y\left(\dot{t} e^{2 v}+\dot{x} e^{2 \lambda}\right)+e^{2(t+f(t+x))}\left\{\frac{\dot{y}}{2}\left(z^{2}-y^{2}+\zeta\right)-y z \dot{z}\right\}\right]$

$\frac{s^{2}}{2} L-s\left[C_{1}(t, x) \dot{t} e^{2 v}+C_{2}(t, x) \dot{x} e^{2 \lambda}\right]+A_{1}$

$s L-\left[C_{1}(t, x) \dot{t} e^{2 v}+C_{2}(t, x) \dot{x} e^{2 \lambda}\right]$

TABLE 19: Conserved quantities corresponding to each Noether symmetry.

\begin{tabular}{ll}
\hline Generator; gauge term: $A\left(s, q^{i}\right)$ & Conserved quantity: $\varphi\left(s, q^{i}, \dot{q}^{i}\right)$ \\
\hline $\mathbf{X}_{0}=-\partial_{t}+\partial_{x}+y \partial_{y}+z \partial_{z}$ & $2\left[\dot{t} e^{2 v}+\dot{x} e^{2 \lambda}+(y \dot{y}+z \dot{z}) e^{2(t+f(t+x))}\right]$ \\
$\mathbf{X}_{4}=-z \partial_{t}+z \partial_{x}+z y \partial_{y}+\frac{1}{2}\left(z^{2}-y^{2}-\sigma\right) \partial_{z}$ & $2\left[z\left(\dot{t} e^{2 v}+\dot{x} e^{2 \lambda}\right)+e^{-2 x / a}\left\{y \dot{y} z-\frac{\dot{z}}{2}\left(z^{2}-y^{2}+\sigma\right)\right\}\right]$ \\
$\mathbf{X}_{5}=y \partial_{t}-y \partial_{x}+\frac{1}{2}\left(z^{2}-y^{2}+\sigma\right) \partial_{y}+y z \partial_{z}$ & $2\left[-y\left(\dot{t} e^{2 v}+\dot{x} e^{2 \lambda}\right)+e^{-2 x / a}\left\{\frac{\dot{y}}{2}\left(z^{2}-y^{2}-\sigma\right)-y z \dot{z}\right\}\right]$ \\
$\mathbf{Y}_{1}=\frac{s^{2}}{2} \partial_{s}+\frac{s}{2}\left[\left(\frac{\dot{\lambda}+1}{\dot{\lambda}}\right) \partial_{t}-\partial_{x}\right] ;$ & $\frac{s^{2}}{2} L-s\left[\left(\frac{\dot{\lambda}+1}{\dot{\lambda}}\right) \dot{t} e^{2 v}+\dot{x} e^{2 \lambda}\right]+A_{1}$ \\
$A_{1}=\int e^{2 v}\left(\frac{\dot{\lambda}+1}{\dot{\lambda}}\right) d t+\int e^{2 \lambda} d x$ & $s L-\left(\frac{\dot{\lambda}+1}{\dot{\lambda}}\right) \dot{t} e^{2 v}-\dot{x} e^{2 \lambda}$ \\
$\mathbf{Y}_{2}=s \partial_{s}+\frac{1}{2}\left[\left(\frac{\dot{\lambda}+1}{\dot{\lambda}}\right) \partial_{t}-\partial_{x}\right]$ & \\
\hline
\end{tabular}

TABLE 20: Conserved quantities corresponding to each Noether symmetry.

\begin{tabular}{ll}
\hline Generator; gauge term: $A\left(s, q^{i}\right)$ & Conserved quantity: $\varphi\left(s, q^{i}, \dot{q}^{i}\right)$ \\
\hline $\mathbf{X}_{0}=-\partial_{t}+\partial_{x}+y \partial_{y}+z \partial_{z}$ & $2\left[\dot{t} e^{2 \gamma}+\dot{x} e^{2 \lambda}+(y \dot{y}+z \dot{z}) e^{2(t+f(t+x))}\right]$ \\
$\mathbf{X}_{4}=-z \partial_{t}+z \partial_{x}+z y \partial_{y}+\frac{1}{2}\left[\left(z^{2}-y^{2}\right)-\psi\right] \partial_{z}$ & $2\left[z\left(\dot{t} e^{2 v}+\dot{x} e^{2 \lambda}\right)+e^{2 t / a}\left\{y \dot{y} z-\frac{\dot{z}}{2}\left(z^{2}-y^{2}+\psi\right)\right\}\right]$ \\
$\mathbf{X}_{5}=y \partial_{t}-y \partial_{x}+\frac{1}{2}\left[\left(z^{2}-y^{2}\right)+\psi\right] \partial_{y}+y z \partial_{z}$ & $2\left[-y\left(\dot{t} e^{2 \nu}+\dot{x} e^{2 \lambda}\right)+e^{2 t / a}\left\{\frac{\dot{y}}{2}\left(z^{2}-y^{2}-\psi\right)-y z \dot{z}\right\}\right]$ \\
$\mathbf{Y}_{1}=\frac{s^{2}}{2} \partial_{s}+\frac{s}{2}\left[\partial_{t}+\left(\frac{1-\dot{v}}{\dot{v}}\right) \partial_{x}\right] ;$ & $\frac{s^{2}}{2} L-s\left[\dot{t} e^{2 v}-\left(\frac{1-\dot{v}}{\dot{v}}\right) \dot{x} e^{2 \lambda}\right]+A_{1}$ \\
$A_{1}=\int e^{2 v} d t-\int e^{2 \lambda}\left(\frac{1-\dot{v}}{\dot{v}}\right) d x$ & $s L-\dot{t} e^{2 v}+\left(\frac{1-\dot{v}}{\dot{v}}\right) \dot{x} e^{2 \lambda}$ \\
$\mathbf{Y}_{2}=s \partial_{s}+\frac{1}{2}\left[\partial_{t}+\left(\frac{1-\dot{v}}{\dot{v}}\right) \partial_{x}\right]$ &
\end{tabular}

$R_{i j}$

$$
\begin{aligned}
& R_{00}=\frac{2}{a^{2}}, \\
& R_{22}=R_{33}=\frac{2}{b^{2}} .
\end{aligned}
$$

$R_{i}^{i}$

$$
R=\frac{6}{x^{2}}
$$

$T_{i j}$

$$
\begin{aligned}
& \kappa T_{00}=-\frac{1}{a^{2}}, \\
& \kappa T_{11}=\frac{3}{x^{2}}, \\
& \kappa T_{22}=\kappa T_{33}=\frac{1}{b^{2}} .
\end{aligned}
$$

$T_{i}^{i}$

$$
T=-\frac{6}{x^{2}} .
$$


Case 2.

$$
\begin{aligned}
d s^{2} & =\left(\frac{x}{a}\right)^{2}\left(d t^{2}-d y^{2}-d z^{2}\right)-d x^{2}, \quad(a \neq 0) . \\
& C_{i j}^{k} \\
C_{13}^{2} & =C_{04}^{3}=C_{05}^{2}=C_{14}^{5}=C_{34}^{0}=C_{25}^{0}=C_{\widehat{0} \hat{1}}^{\hat{1}}=C_{\widehat{0} \hat{2}}^{\hat{0}} \\
& =1, \\
C_{13}^{2} & =C_{15}^{4}=C_{45}^{1}=C_{\hat{1} 2}^{\hat{1}}=-1 . \\
& R_{i j k l}
\end{aligned}
$$$$
\begin{aligned}
& R_{0202}=R_{0303}=-\frac{1}{a^{2}}\left(\frac{x}{a}\right)^{2}, \\
& R_{2323}=\frac{1}{a^{2}}\left(\frac{x}{a}\right)^{2} .
\end{aligned}
$$

$R_{i j}$

$$
\begin{aligned}
& R_{00}=\frac{2}{a^{2}}, \\
& R_{22}=R_{33}=\frac{2}{a^{2}} .
\end{aligned}
$$

$R_{i}^{i}$

$$
R=\frac{6}{x^{2}} .
$$

$$
\begin{aligned}
& T_{i j} \\
& \kappa T_{00}=-\frac{1}{a^{2}}, \\
& \kappa T_{11}=\frac{3}{x^{2}}, \\
& \kappa T_{22}=\kappa T_{33}=\frac{1}{a^{2}} .
\end{aligned}
$$

$T^{i}$

$$
T=-\frac{6}{x^{2}} .
$$

Case 3.

$$
\begin{aligned}
& d s^{2}=d t^{2}-\left(\frac{t}{a}\right)^{2}\left(d x^{2}+d y^{2}+d z^{2}\right), \quad(a \neq 0) . \\
& C_{i j}^{k} \\
& C_{12}^{3}=C_{04}^{3}=C_{05}^{2}=C_{15}^{4}=C_{\widehat{12}}^{\hat{1}}=-1, \\
& C_{13}^{2}=C_{14}^{5}=C_{25}^{0}=C_{34}^{0}=C_{45}^{1}=C_{\widehat{01}}^{\widehat{1}}=C_{\widehat{02}}^{\widehat{0}}=1 .
\end{aligned}
$$

$$
R_{i j k l}
$$

$$
\begin{aligned}
& R_{1212}=R_{1313}=-\frac{1}{a^{2}}\left(\frac{t}{a}\right)^{2} \\
& R_{2323}=-\frac{1}{a^{2}}\left(\frac{t}{a}\right)^{2}
\end{aligned}
$$

$R_{i j}$

$$
R_{00}=R_{22}=R_{33}=\frac{2}{a^{2}} .
$$

$R_{i}^{i}$

$$
R=-\frac{6}{t^{2}}
$$

$T_{i j}$

$$
\begin{aligned}
& \kappa T_{00}=\frac{3}{t^{2}}, \\
& \kappa T_{11}=-\frac{1}{a^{2}}, \\
& \kappa T_{22}=\kappa T_{33}=-\frac{1}{a^{2}} .
\end{aligned}
$$

$T_{i}^{i}$

$$
T=\frac{6}{t^{2}} .
$$

Case 4.

$$
\begin{aligned}
d s^{2}= & e^{2 \nu(t+x)} d t^{2}-e^{2 \lambda(t+x)} d x^{2} \\
& -e^{2[t+f(t+x)]}\left(d y^{2}+d z^{2}\right),
\end{aligned}
$$

subject to the constraint $\zeta=2 \int e^{2(\gamma(t+x)-t-f(t+x))}=$ $e^{2(v(t+x)-t-f(t+x))}-e^{2(\lambda(t+x)-t-f(t+x))}$. We denote $C_{i}(t, x)=1+$ $2 f_{i}(t+x)$, where $(i=1,2)$ in Table 18. For metrics (122), (129) and (135), we substitute $\alpha=\left(\lambda^{\prime \prime}+\lambda^{\prime 2}-\nu^{\prime} \lambda^{\prime}\right)$ and $\beta=\left(\nu^{\prime \prime}+\nu^{\prime 2}-\nu^{\prime} \lambda^{\prime}\right)$ for simplicity in the components of relevant tensors.

$$
\begin{aligned}
& C_{i j}^{k} \\
C_{13}^{2} & =C_{14}^{5}=C_{24}^{0}=C_{25}^{1}=C_{34}^{1}=C_{04}^{4}=C_{05}^{5}=C_{\widehat{0} \hat{1}}^{\hat{2}} \\
& =C_{\widehat{0} \hat{2}}^{\hat{0}}=1, \\
C_{12}^{3} & =C_{02}^{2}=C_{03}^{3}=C_{15}^{4}=C_{35}^{0}=C_{\widehat{1} 2}^{\hat{1}}=-1 .
\end{aligned}
$$




$$
\begin{aligned}
& R_{i j k l} \\
& R_{0101}=\alpha e^{2 \lambda}-\beta e^{2 \nu}, \\
& R_{0202}=R_{0303}=e^{2 \mu}\left[f^{\prime \prime}+\left(1+f^{\prime}\right)\left(1+f^{\prime}-\nu^{\prime}\right)\right. \\
& \left.-e^{2(v-\lambda)} v^{\prime} f^{\prime}\right] \text {, } \\
& R_{0212}=R_{0313}=e^{2 \mu}\left[f^{\prime \prime}+\left(1+f^{\prime}\right)\left(f^{\prime}-v^{\prime}\right)\right. \\
& \left.-\lambda^{\prime} f^{\prime}\right] \\
& R_{2323}=e^{4(t+f(t+x))}\left[f^{\prime 2} e^{-2 \lambda}-\left(1+f^{\prime}\right)^{2} e^{-2 \nu}\right], \\
& R_{1212}=R_{1313}=e^{2(t+f(t+x))}\left[f^{\prime \prime}-f^{\prime}\left(\lambda^{\prime}-f^{\prime}\right)\right. \\
& \left.-\lambda^{\prime}\left(1+f^{\prime}\right) e^{2(\lambda-v)}\right] \text {. } \\
& R_{i j} \\
& R_{00}=e^{2(\nu-\lambda)}\left(\beta+2 v^{\prime} f^{\prime}\right)-\left(\alpha+2 f^{\prime \prime}+2\left(1+f^{\prime}\right)^{2}\right. \\
& \left.-2 v^{\prime} f^{\prime}\right) \\
& R_{01}=-2\left[f^{\prime \prime}+\left(1+f^{\prime}\right)\left(f^{\prime}-\nu^{\prime}\right)-\lambda^{\prime} f^{\prime}\right], \\
& R_{22}=R_{33}=e^{2(t+f(t+x))}\left[e ^ { - 2 v } \left\{f^{\prime \prime}\right.\right. \\
& \left.+\left(1+f^{\prime}\right)\left(\lambda^{\prime}+2\left(1+f^{\prime}\right)-\nu^{\prime}\right)\right\}-e^{-2 \lambda}\left\{f^{\prime \prime}\right. \\
& \left.\left.+f^{\prime}\left(\nu^{\prime}+2 f^{\prime}-\lambda^{\prime}\right)\right\}\right] \\
& R_{11}=e^{2(\lambda-\nu)}\left[\alpha+2 \lambda^{\prime}\left(1+f^{\prime}\right)\right]-\left[\beta+2\left(f^{\prime \prime}\right.\right. \\
& \left.\left.-\lambda^{\prime} f^{\prime}+f^{\prime 2}\right)\right] \text {. } \\
& R_{i}^{i} \\
& R=2 e^{-2 \lambda}\left[2 f^{\prime \prime}+f^{\prime}\left(2 v^{\prime}-2 \lambda^{\prime}+3 f^{\prime}\right)+\beta\right] \\
& -2 e^{-2 v}\left[2 f^{\prime \prime}-\left(1+f^{\prime}\right)\left(2 v^{\prime}-3\left(1+f^{\prime}\right)-2 \lambda^{\prime}\right)\right. \\
& +\alpha] \text {. } \\
& T_{i j} \\
& \kappa T_{00}=e^{2(\nu-\lambda)}\left(2 \lambda^{\prime} f^{\prime}-2 f^{\prime \prime}-3 f^{\prime 2}\right)+\left(1+f^{\prime}\right)(1 \\
& \left.+f^{\prime}+2 \lambda^{\prime}\right) \text {, } \\
& \kappa T_{01}=R_{01} \text {, } \\
& \kappa T_{11}=e^{2(\lambda-v)}\left[\left(1+f^{\prime}\right)\left(2 v^{\prime}-3-3 f^{\prime}\right)-2 f^{\prime \prime}\right] \\
& +f^{\prime}\left(f^{\prime}+2 v^{\prime}\right) \text {, }
\end{aligned}
$$

$$
\begin{aligned}
& \kappa T_{22}=\kappa T_{33} \\
& \quad=e^{2(t+f(t+x))}\left[e^{-2 \lambda}\left(f^{\prime \prime}+f^{\prime}\left(v^{\prime}-\lambda^{\prime}+f^{\prime}\right)+\beta\right)\right. \\
& \left.+e^{-2 v}\left(f^{\prime \prime}-\left(1+f^{\prime}\right)\left(v^{\prime}-\lambda^{\prime}-f^{\prime}-1\right)+\alpha\right)\right] .
\end{aligned}
$$

$T^{i}{ }_{i}$

$$
\begin{aligned}
T & =-2 e^{-2 \lambda}\left[2 f^{\prime \prime}+f^{\prime}\left(2 \nu^{\prime}-2 \lambda^{\prime}+3 f^{\prime}\right)+\beta\right] \\
& +2 e^{-2 \nu}\left[2 f^{\prime \prime}-\left(1+f^{\prime}\right)\left(2 \nu^{\prime}-3\left(1+f^{\prime}\right)-2 \lambda^{\prime}\right)\right. \\
& +\alpha] .
\end{aligned}
$$

Case 5.

$$
\begin{array}{r}
d s^{2}=e^{2 \gamma(t+x)} d t^{2}-e^{2 \lambda(t+x)} d x^{2}-e^{-2 x / a}\left(d y^{2}+d z^{2}\right), \\
(a \neq 0)
\end{array}
$$

subject to the constraint $\sigma=2 \int e^{2(\nu+x / a)} d t=e^{2(\lambda+x / a)}-$ $e^{2(v+x / a)}$.

$R_{a b c d}$

$$
\begin{aligned}
& R_{0101}=\alpha e^{2 \lambda}-\beta e^{2 \nu} \\
& R_{0202}=R_{0303}=\frac{1}{a} \nu^{\prime} e^{2(v-\lambda-x / a)}, \\
& R_{0212}=R_{0313}=\frac{1}{a} \lambda^{\prime} \\
& R_{1212}=R_{1313}=\frac{1}{a}\left(\frac{1}{a}-\lambda^{\prime}\right) e^{-2 x / a}, \\
& R_{2323}=\frac{1}{a^{2}} e^{-2(2 x / a+\lambda)}
\end{aligned}
$$

$$
\begin{aligned}
& R_{a b} \\
& R_{00}=e^{2(\nu-\lambda)}\left(\beta-\frac{2}{a} \nu^{\prime}\right)-\alpha, \\
& R_{01}=-\frac{2}{a} \lambda^{\prime}, \\
& R_{11}=\alpha e^{2(\lambda-\nu)}-\left(\beta-\frac{2}{a^{2}}+\frac{2}{a} \lambda^{\prime}\right), \\
& R_{22}=R_{33}=-\frac{1}{a}\left[\left(\lambda^{\prime}-\nu^{\prime}\right)+\frac{2}{a}\right] e^{-2(x / a+\lambda)} .
\end{aligned}
$$

$R_{i}^{i}$

$$
R=2 e^{-2 \lambda}\left(\frac{3}{a^{2}}-\frac{2}{a}\left(\nu^{\prime}-\lambda^{\prime}\right)+\beta\right)-2 \alpha e^{-2 \nu} .
$$




$$
\begin{aligned}
& T_{a b} \\
& \kappa T_{00}=-\frac{1}{a}\left(2 \lambda^{\prime}+\frac{3}{a}\right) e^{2(\nu-\lambda)}, \\
& \kappa T_{01}=R_{01}, \\
& \kappa T_{11}=\frac{1}{a}\left(\frac{1}{a}-2 \nu^{\prime}\right), \\
& \kappa T_{22}= \kappa T_{33} \\
&= e^{-2(x / a+\lambda)}\left(\frac{1}{a^{2}}-\frac{1}{a}\left(\nu^{\prime}-\lambda^{\prime}\right)+\beta\right) \\
&-\alpha e^{-2(x / a+v)} .
\end{aligned}
$$

$T$

$T=-2 e^{-2 \lambda}\left(\frac{3}{a^{2}}-\frac{2}{a}\left(\nu^{\prime}-\lambda^{\prime}\right)+\beta\right)+2 \alpha e^{-2 \nu}$.

Case 6.

$$
\begin{array}{r}
d s^{2}=e^{2 v(t+x)} d t^{2}-e^{2 \lambda(t+x)} d x^{2}-e^{2 t / a}\left(d y^{2}+d z^{2}\right) \\
(a \neq 0)
\end{array}
$$

subject to the constraint $\psi=2 \int e^{2(\lambda-t / a)} d t=e^{2(\lambda-t / a)}-$ $e^{2(v-t / a)}$.

$$
\begin{aligned}
& R_{i j k l} \\
& R_{0101}=\alpha e^{2 \lambda}-\beta e^{2 \nu} \\
& R_{0202}=R_{0303}=-\frac{1}{a}\left(v^{\prime}-\frac{1}{a}\right) e^{2 t / a}, \\
& R_{0212}=R_{0313}=-\frac{1}{a} v^{\prime} e^{2 t / a} \text {, } \\
& R_{1212}=R_{1313}=\frac{1}{a} \lambda^{\prime} e^{2(t / a-\nu+\lambda)} \\
& R_{2323}=-\frac{1}{a^{2}} e^{2(2 t / a-v)} . \\
& R_{i j} \\
& R_{00}=\beta e^{2(\nu-\lambda)}-\left(\alpha+\frac{2}{a^{2}}-\frac{2}{a} \nu^{\prime}\right), \\
& R_{01}=\frac{2}{a} v^{\prime}, \\
& R_{11}=e^{2(\lambda-\nu)}\left(\alpha+\frac{2}{a} \lambda^{\prime}\right)-\beta, \\
& R_{22}=R_{33}=\frac{1}{a}\left[\frac{2}{a}+\left(\lambda^{\prime}-\nu^{\prime}\right)\right] e^{2(t / a-v)} . \\
& R=2 \beta e^{-2 \lambda}-2 e^{-2 \nu}\left(\frac{3}{a^{2}}-\frac{2}{a}\left(\nu^{\prime}-\lambda^{\prime}\right)+\alpha\right) .
\end{aligned}
$$

TABLE 21: Conserved quantities corresponding to each Noether symmetry.

\begin{tabular}{ll}
\hline $\begin{array}{l}\text { Generator; gauge term: } \\
A\left(s, q^{i}\right)\end{array}$ & Conserved quantity: $\varphi\left(s, q^{i}, \dot{q}^{i}\right)$ \\
\hline $\mathbf{X}_{0}=\partial_{t}$ & $-2\left(\frac{x}{a}\right)^{2 b} \dot{t}$ \\
$\mathbf{Y}_{1}=s \partial_{y} ; A_{1}=-2 y$ & $2(y-s \dot{y})$ \\
$\mathbf{Y}_{2}=s \partial_{z} ; A_{2}=-2 z$ & $2(z-s \dot{z})$ \\
$\mathbf{Y}_{3}=s \partial_{s}+(1-b) \frac{t}{2} \partial_{t}+$ & $s L-2(1-b)\left(\frac{x}{a}\right)^{2 b} t \dot{t}+x \dot{x}+y \dot{y}+z \dot{z}$ \\
$\frac{x}{2} \partial_{x}+\frac{y}{2} \partial_{y}+\frac{z}{2} \partial_{z}$ & \\
\hline
\end{tabular}

TABLE 22: Conserved quantities corresponding to each Noether symmetry.

\begin{tabular}{ll}
\hline Generator; gauge term: $A\left(s, q^{i}\right)$ & $\begin{array}{l}\text { Conserved quantity: } \\
\varphi\left(s, q^{i}, \dot{q}^{i}\right)\end{array}$ \\
\hline $\mathbf{X}_{0}=\partial_{x}$ & $2\left(\frac{t}{a}\right)^{2 b} \dot{x}$ \\
$\mathbf{Y}_{1}=s \partial_{y} ;$ & $2(y-s \dot{y})$ \\
$A_{1}=-2 y$ & \\
$\mathbf{Y}_{2}=s \partial_{z} ;$ & $2(z-s \dot{z})$ \\
$A_{2}=-2 z$ & $s L-t \dot{t}+(1-b)\left(\frac{t}{b}\right)^{2} x \dot{x}+$ \\
$\mathbf{Y}_{3}=s \partial_{s}+\frac{1}{2}\left(t \partial_{t}+y \partial_{y}+z \partial_{z}\right)+$ & $y \dot{y}+z \dot{z}$ \\
$\frac{(1-b)}{2} x \partial_{x}$ & \\
\hline
\end{tabular}

$$
\begin{aligned}
& T_{i j} \\
& \kappa T_{00}=\frac{1}{a}\left(2 \lambda^{\prime}+\frac{1}{a}\right), \\
& \kappa T_{01}=R_{01} \text {, } \\
& \kappa T_{11}=\frac{1}{a}\left(2 \nu^{\prime}-\frac{3}{a}\right) e^{2(\nu-\lambda)}, \\
& \kappa T_{22}=\kappa T_{33} \\
& =\beta e^{2(t / a-\lambda)}-e^{2(t / a-v)}\left(\frac{1}{a^{2}}-\frac{1}{a}\left(\nu^{\prime}-\lambda^{\prime}\right)+\alpha\right) . \\
& T_{i}^{i} \\
& T=-2 e^{-2 \lambda}\left(v^{\prime \prime}+v^{\prime 2}-v^{\prime} \lambda^{\prime}\right) \\
& +2 e^{-2 v}\left(\frac{3}{a^{2}}-\frac{2}{a}\left(v^{\prime}-\lambda^{\prime}\right)+\lambda^{\prime \prime}+\lambda^{\prime 2}-v^{\prime} \lambda^{\prime}\right) .
\end{aligned}
$$

The structure constants of the corresponding Lie algebra for (129) and (135) are the same as for (122).

4.4. Eight Noether Symmetries. All metrics that appear in this section admit eight Noether symmetries, out of which four are given in (7) and four are presented in Tables 21-28, respectively. In addition, we have also calculated the structure constants of the associated Lie algebra and the nonzero components of the relevant tensors. 
TABLE 23: Conserved quantities corresponding to each Noether symmetry.

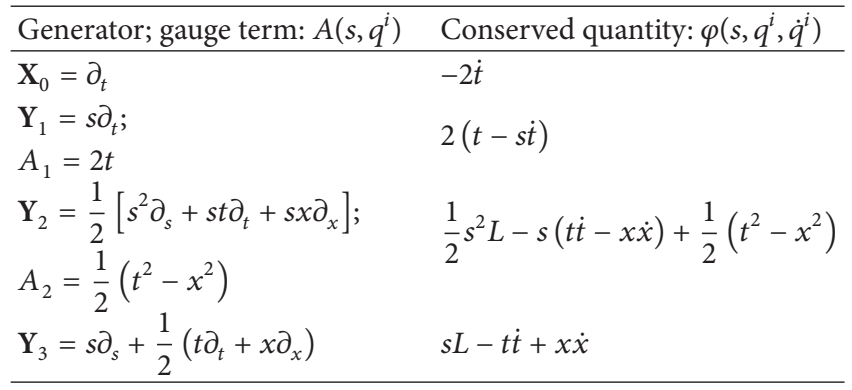

Case 1.

$$
\begin{aligned}
& d s^{2}=\left(\frac{x}{a}\right)^{2 b} d t^{2}-d x^{2}-d y^{2}-d z^{2}, \\
& (a \neq 0, b \neq 0,1) \text {. } \\
& C_{i j}^{k} \\
& C_{13}^{2}=C_{1 \widehat{1}}^{\hat{2}}=C_{1 \widehat{2}}^{\hat{1}}=C_{\widehat{0} \hat{1}}^{3}=C_{\widehat{0} \hat{2}}^{2}=C_{\widehat{0} \widehat{3}}^{\hat{0}}=1 \text {, } \\
& C_{\widehat{1} \widehat{3}}^{\hat{1}}=C_{\widehat{2} \widehat{3}}^{\widehat{2}}=-\frac{1}{2} \text {, } \\
& C_{2 \widehat{3}}^{2}=C_{3 \widehat{3}}^{3}=\frac{1}{2} \text {, } \\
& C_{12}^{3}=-1 \text {, } \\
& C_{0 \widehat{3}}^{0}=\frac{1-b}{2} \text {. } \\
& R_{i j k l} \\
& R_{0101}=\frac{b(b-1)}{x^{2}}\left(\frac{x}{a}\right)^{2 b} \\
& R_{i j} \\
& R_{00}=\frac{b(b-1)}{x^{2}}\left(\frac{x}{a}\right)^{2 b}, \\
& R_{11}=\frac{b(1-b)}{x^{2}} \text {. } \\
& R=2 \frac{b(b-1)}{x^{2}}
\end{aligned}
$$

$T_{i j}$

$$
\kappa T_{22}=\kappa T_{33}=\frac{b(b-1)}{x^{2}}
$$

$T_{i}^{i}$

$$
T=-2 \frac{b(b-1)}{x^{2}}
$$

Metric (141) is a gravitational wave solution when $R_{i j} \rightarrow 0$ as $x \rightarrow \infty$ provided $b<1$ and $b \neq 0$.

Case 2.

$$
\begin{aligned}
d s^{2}=d t^{2}-\left(\frac{t}{a}\right)^{2 b} d x^{2}-d y^{2}-d z^{2}, & \\
& (b \neq 0,1, a \neq 0) .
\end{aligned}
$$

The structure constants of the corresponding Lie algebra are the same as for (141).

$$
R_{i j k l}
$$

$$
R_{0101}=\frac{b(b-1)}{t^{2}}\left(\frac{t}{a}\right)^{2 b}
$$

$R_{i j}$

$$
\begin{aligned}
& R_{00}=\frac{b(1-b)}{t^{2}}, \\
& R_{11}=\frac{b(b-1)}{t^{2}}\left(\frac{t}{a}\right)^{2 b} .
\end{aligned}
$$

$R_{i}^{i}$

$$
R=-2 \frac{b(b-1)}{t^{2}}
$$

$T_{i j}$

$$
\kappa T_{22}=\kappa T_{33}=-\frac{b(b-1)}{t^{2}} .
$$

$T_{i}^{i}$

$$
T=2 \frac{b(b-1)}{t^{2}}
$$

Metric (148) is a gravitational wave solution $\left(R_{i j} \rightarrow 0\right.$ as $t \rightarrow \infty)$ provided $b<1$ and $b \neq 0$.

Case 3.

$$
d s^{2}=d t^{2}-d x^{2}-\left(\frac{x}{K}\right)^{2}\left(d y^{2}+d z^{2}\right), \quad(K \neq 0) .
$$

$$
C_{i j}^{k}
$$

$$
\begin{aligned}
& C_{13}^{2}=C_{\widehat{01}}^{0}=C_{\widehat{02}}^{\widehat{3}}=C_{\widehat{0} \hat{3}}^{\hat{0}}=1, \\
& C_{12}^{3}=C_{\widehat{2} \hat{3}}^{\widehat{2}}=-1, \\
& C_{\widehat{1} \widehat{1}}^{\widehat{1}}=-\frac{1}{2} .
\end{aligned}
$$

$$
R_{i j k l}
$$

$$
R_{2323}=\left(\frac{x}{K^{2}}\right)^{2}
$$


TABLE 24: Conserved quantities corresponding to each Noether symmetry.

Generator; gauge term: $A\left(s, q^{i}\right)$

Conserved quantity: $\varphi\left(s, q^{i}, \dot{q}^{i}\right)$

$\mathbf{X}_{0}=\partial_{t}$

$-2\left(\frac{x}{a}\right)^{2} \dot{t}$

$\mathbf{X}_{4}=y \partial_{t}+\left(\frac{K}{a}\right)^{2} t \partial_{y}$

$2\left(\frac{x}{a}\right)^{2}(y \dot{t}-t \dot{y})$

$\mathbf{X}_{5}=z \partial_{t}+\left(\frac{K}{a}\right)^{2} t \partial_{z}$

$2\left(\frac{x}{a}\right)^{2}(z \dot{t}-t \dot{z})$

$\mathbf{Y}_{1}=s \partial_{s}+\frac{1}{4}\left(t \partial_{t}+x \partial_{x}+y \partial_{y}+z \partial_{z}\right)$

$s L-\frac{1}{2}\left[\left(\frac{x}{a}\right)^{2} t \dot{t}-\left(\frac{x}{b}\right)^{2} x \dot{x}-\left(\frac{x}{K}\right)^{2}(y \dot{y}+z \dot{z})\right]$

TABLE 25: Conserved quantities corresponding to each Noether symmetry.

Generator; gauge term: $A\left(s, q^{i}\right)$

$\mathbf{X}_{0}=\partial_{t}$

$\mathbf{X}_{4}=y \partial_{t}+\left(\frac{K}{a}\right)^{2} t \partial_{y}$

$\mathbf{X}_{5}=z \partial_{t}+\left(\frac{K}{a}\right)^{2} t \partial_{z}$

$\mathbf{Y}_{1}=s \partial_{s}+\frac{b}{2 b+2}\left(t \partial_{t}+\frac{1}{b} x \partial_{x}+y \partial_{y}+z \partial_{z}\right)$

TABLE 26: Conserved quantities corresponding to each Noether symmetry.

\begin{tabular}{ll}
\hline $\begin{array}{l}\text { Generator; gauge term: } \\
A\left(s, q^{i}\right)\end{array}$ & $\begin{array}{l}\text { Conserved quantity: } \\
\varphi\left(s, q^{i}, \dot{q}^{i}\right)\end{array}$ \\
\hline $\mathbf{X}_{0}=\partial_{t}$ & $-2\left(\frac{x}{a}\right)^{2 b} \dot{t}$ \\
$\mathbf{X}_{4}=y \partial_{t}+t \partial_{y}$ & $2\left(\frac{x}{a}\right)^{2 b}(y \dot{t}-t \dot{y})$ \\
$\mathbf{X}_{5}=z \partial_{t}+t \partial_{z}$ & $2\left(\frac{x}{a}\right)^{2 b}(z \dot{t}-t \dot{z})$ \\
$\mathbf{Y}_{1}=s \partial_{s}+$ & $s L+x \dot{x}-$ \\
$\frac{1-b}{2}\left(t \partial_{t}+y \partial_{y}+z \partial_{z}\right)+\frac{x}{2} \partial_{x}$ & $(1-b)\left(\frac{x}{a}\right)^{2 b}(t \dot{t}-y \dot{y}-z \dot{z})$ \\
\hline
\end{tabular}

TABLE 27: Conserved quantities corresponding to each Noether symmetry.

\begin{tabular}{ll}
\hline $\begin{array}{l}\text { Generator; gauge term: } \\
A\left(s, q^{i}\right)\end{array}$ & $\begin{array}{l}\text { Conserved quantity: } \\
\varphi\left(s, q^{i}, \dot{q}^{i}\right)\end{array}$ \\
\hline $\mathbf{X}_{0}=\partial_{x}$ & $2\left(\frac{t}{a}\right)^{2 b} \dot{x}$ \\
$\mathbf{X}_{4}=y \partial_{x}-x \partial_{y}$ & $2\left(\frac{t}{a}\right)^{2 b}(y \dot{x}-x \dot{y})$ \\
$\mathbf{X}_{5}=z \partial_{x}-x \partial_{z}$ & $2\left(\frac{t}{a}\right)^{2 b}(z \dot{x}-x \dot{z})$ \\
$\mathbf{Y}_{1}=s \partial_{s}+\frac{t}{2} \partial_{t}+$ & $s L-t \dot{t}+$ \\
$\frac{1-b}{2}\left(x \partial_{x}+y \partial_{y}+z \partial_{z}\right)$ & $(1-b)\left(\frac{t}{a}\right)^{2 b}(x \dot{x}+y \dot{y}+z \dot{z})$ \\
\hline
\end{tabular}

Conserved quantity: $\varphi\left(s, q^{i}, \dot{q}^{i}\right)$

$-2\left(\frac{x}{a}\right)^{2} \dot{t}$

$2\left(\frac{x}{a}\right)^{2}(y \dot{t}-t \dot{y})$

$2\left(\frac{x}{a}\right)^{2}(z \dot{t}-t \dot{z})$

$s L-\frac{b}{b+1}\left[\left(\frac{x}{a}\right)^{2} t \dot{t}-\left(\frac{c}{x}\right)^{2 b} \frac{x}{b} \dot{x}-\left(\frac{x}{K}\right)^{2}(y \dot{y}+z \dot{z})\right]$

TABLE 28: Conserved quantities corresponding to each Noether symmetry.

\begin{tabular}{ll}
\hline Generator; gauge term: & Conserved quantity: $\varphi\left(s, q^{i}, \dot{q}^{i}\right)$ \\
\hline $\begin{array}{l}\mathbf{X}_{0}=\partial_{t} \pm \partial_{x} \\
\mathbf{Y}_{1}=s\left(\partial_{t} \pm \partial_{x}\right) ;\end{array}$ & $2(\dot{t} \pm \dot{x})$ \\
$A_{1}=2(t \pm x)$ & $2[s(-\dot{t} \pm \dot{x})+t \pm x]$ \\
$\mathbf{Y}_{2}=\frac{1}{2}\left(s^{2} \partial_{s}+s t \partial_{t}+s x \partial_{x}\right) ;$ & $\frac{1}{2}\left[s^{2} L-2 s(t \dot{t}-x \dot{x})-\left(t^{2}-x^{2}\right)\right]$ \\
$A_{1}=\frac{1}{2}\left(t^{2}-x^{2}\right)$ & \\
$\mathbf{Y}_{3}=s \partial_{s}+\frac{1}{2}\left(t \partial_{t}+x \partial_{x}\right)$ & $s L-t \dot{t}+x \dot{x}$
\end{tabular}

$R_{i}^{i}$

$$
R=\frac{2}{x^{2}}
$$

$T_{i j}$

$$
\begin{aligned}
& \kappa T_{00}=-\frac{1}{x^{2}}, \\
& \kappa T_{11}=\frac{1}{x^{2}} .
\end{aligned}
$$

$T_{i}^{i}$

$$
T=-\frac{2}{x^{2}} .
$$

Case 4.

$$
\begin{array}{r}
d s^{2}=\left(\frac{x}{a}\right)^{2} d t^{2}-\left(\frac{x}{b}\right)^{2} d x^{2}-\left(\frac{x}{K}\right)^{2}\left(d y^{2}+d z^{2}\right), \\
(a \neq b \neq K \neq 0) .
\end{array}
$$


$C_{i j}^{k}$

$$
\begin{aligned}
& C_{13}^{2}=C_{14}^{5}=C_{25}^{0}=C_{34}^{0}=C_{\widehat{0} \hat{1}}^{0}=1, \\
& C_{12}^{3}=C_{15}^{4}=-1, \\
& C_{04}^{3}=C_{05}^{2}=\left(\frac{K}{a}\right)^{2}, \\
& C_{45}^{1}=-\left(\frac{K}{a}\right)^{2}, \\
& C_{0 \widehat{1}}^{0}=C_{2 \widehat{1}}^{2}=C_{3 \widehat{1}}^{3}=\frac{1}{4} .
\end{aligned}
$$

$R_{i j k l}$

$$
\begin{aligned}
& R_{0101}=\frac{1}{a^{2}}, \\
& R_{0202}=R_{0303}=-\left(\frac{b}{a K}\right)^{2}, \\
& R_{1212}=R_{1313}=-\frac{1}{K^{2}}, \\
& R_{2323}=\left(\frac{b}{K^{2}}\right)^{2} .
\end{aligned}
$$

$T_{i j}$

$$
\begin{aligned}
& \kappa T_{00}=\left(\frac{b}{a}\right)^{2} \frac{1}{x^{2}}, \\
& \kappa T_{11}=\frac{3}{x^{2}} \\
& \kappa T_{22}=\kappa T_{33}=-\left(\frac{b}{K}\right)^{2} \frac{1}{x^{2}} .
\end{aligned}
$$

$T_{i}^{i}$

$$
T=0 \text {. }
$$

Metric (161) is a gravitational wave solution $\left(R_{i j} \rightarrow 0\right.$ as $x \rightarrow \infty$ ) provided $a, b, K \neq 0$.
Case 5.

(i) $d s^{2}$

$$
\begin{aligned}
= & \left(\frac{x}{a}\right)^{2} d t^{2}-\left(\frac{x}{c}\right)^{2 b} d x^{2} \\
& -\left(\frac{x}{K}\right)^{2}\left(d y^{2}+d z^{2}\right),
\end{aligned}
$$$$
(a \neq b \neq c \neq K \neq 0) .
$$

(ii) $d s^{2}$

$$
\begin{gathered}
=\left(\frac{x}{a}\right)^{2} d t^{2}-\left(\frac{c}{x}\right)^{2 b} d x^{2} \\
-\left(\frac{x}{K}\right)^{2}\left(d y^{2}+d z^{2}\right),
\end{gathered}
$$

$$
(b \neq 0,1, a \neq c \neq K \neq 0) .
$$

$C_{i j}^{k}$

$$
\begin{aligned}
& C_{13}^{2}=C_{14}^{5}=C_{25}^{0}=C_{34}^{0}=C_{\widehat{0} \hat{1}}^{0}=1, \\
& C_{12}^{3}=C_{15}^{4}=-1, \\
& C_{04}^{3}=C_{05}^{2}=\left(\frac{K}{a}\right)^{2}, \\
& C_{45}^{1}=-\left(\frac{K}{a}\right)^{2}, \\
& C_{0 \hat{1}}^{0}=C_{2 \widehat{1}}^{2}=C_{3 \hat{1}}^{3}=\frac{b}{2 b+2} .
\end{aligned}
$$

$R_{i j k l}$

For (i)

$R_{0101}=\frac{b}{a^{2}}$,

$R_{0202}=R_{0303}=-\left(\frac{x}{a K}\right)^{2}\left(\frac{c}{x}\right)^{2 b}$,

$R_{1212}=R_{1313}=-\frac{b}{K^{2}}$,

$R_{2323}=\left(\frac{x}{K^{2}}\right)^{2}\left(\frac{c}{x}\right)^{2 b}$.

For (ii)

$R_{0101}=b\left(\frac{x}{a}\right)^{2}$,

$R_{0202}=R_{0303}=-\frac{1}{K^{2}}\left(\frac{x}{a}\right)^{2}\left(\frac{x}{c}\right)^{2 b}$,

$R_{1212}=R_{1313}=-b\left(\frac{x}{K}\right)^{2}$, 
$R_{i j}$

For (i)

$$
\begin{aligned}
& R_{00}=\left(\frac{2-b}{a^{2}}\right)\left(\frac{c}{x}\right)^{2 b}, \\
& R_{11}=\frac{3 b}{x^{2}}, \\
& R_{22}=R_{33}=-\left(\frac{2-b}{K^{2}}\right)\left(\frac{c}{x}\right)^{2 b} .
\end{aligned}
$$

For (ii)

$$
\begin{aligned}
& R_{00}=\left(\frac{2-b x^{2}}{a^{2}}\right)\left(\frac{x}{c}\right)^{2 b}, \\
& R_{11}=3 b, \\
& R_{22}=R_{33}=-\left(\frac{2-b x^{2}}{K^{2}}\right)\left(\frac{x}{c}\right)^{2 b} .
\end{aligned}
$$

$R_{i}^{i}$

For (i)

$$
R=6\left(\frac{1-b}{x^{2}}\right)\left(\frac{c}{x}\right)^{2 b} .
$$

For (ii)

$$
R=6\left(\frac{1+b}{x^{2}}\right)\left(\frac{x}{c}\right)^{2 b} .
$$

$T_{i j}$

For (i)

$$
\begin{aligned}
& \kappa T_{00}=\left(\frac{2 b-1}{a^{2}}\right)\left(\frac{c}{x}\right)^{2 b}, \\
& \kappa T_{11}=\frac{3}{x^{2}}, \\
& \kappa T_{22}=\kappa T_{33}=-\left(\frac{2 b-1}{K^{2}}\right)\left(\frac{c}{x}\right)^{2 b} .
\end{aligned}
$$

For (ii)

$$
\begin{aligned}
& \kappa T_{00}=\left(\frac{2 b x^{2}-1}{a^{2}}\right)\left(\frac{x}{c}\right)^{2 b}, \\
& \kappa T_{11}=\frac{3}{x^{2}}, \\
& \kappa T_{22}=\kappa T_{33}=-\left(\frac{2 b x^{2}-1}{a^{2}}\right)\left(\frac{x}{c}\right)^{2 b} .
\end{aligned}
$$

$T^{i}{ }_{i}$

For (i)

$$
T=-6\left(\frac{1-b}{x^{2}}\right)\left(\frac{c}{x}\right)^{2 b} .
$$

For (ii)

$$
T=6\left(b-\frac{1}{x^{2}}\right)\left(\frac{x}{c}\right)^{2 b} .
$$

Metric (168)(i) is a gravitational wave solution $\left(R_{i j} \rightarrow 0\right.$ as $x \rightarrow \infty$ ) provided $b>0$.

Case 6.

$$
d s^{2}=\left(\frac{x}{a}\right)^{2 b}\left(d t^{2}-d y^{2}-d z^{2}\right)-d x^{2},
$$

$$
(b \neq 0,1, a \neq 0) \text {. }
$$

$$
C_{i j}^{k}
$$

$$
\begin{aligned}
& C_{13}^{2}=C_{14}^{5}=C_{04}^{3}=C_{05}^{2}=C_{24}^{0}=C_{34}^{0}=C_{\widehat{0} \hat{1}}^{\hat{0}}=1, \\
& C_{12}^{3}=C_{15}^{4}=C_{45}^{1}=-1, \\
& C_{0 \hat{1}}^{0}=C_{2 \hat{1}}^{2}=C_{3 \hat{1}}^{3}=\frac{1-b}{2} .
\end{aligned}
$$

$$
R_{i j k l}
$$

$$
\begin{aligned}
& R_{0101}=-\frac{b(b-1)}{x^{2}}\left(\frac{x}{a}\right)^{2 b}, \\
& R_{0202}=R_{0303}=\left(\frac{b}{x}\right)^{2}\left(\frac{x}{a}\right)^{4 b}, \\
& R_{1212}=R_{1313}=\frac{b(b-1)}{x^{2}}\left(\frac{x}{a}\right)^{2 b}, \\
& R_{2323}=\left(\frac{b}{x}\right)^{2}\left(\frac{x}{a}\right)^{4 b} .
\end{aligned}
$$

$R_{i j}$

$$
\begin{aligned}
& R_{00}=\frac{b(3 b-1)}{x^{2}}\left(\frac{x}{a}\right)^{2 b}, \\
& R_{11}=3 \frac{b(1-b)}{x^{2}}, \\
& R_{22}=R_{33}=\frac{b(1-3 b)}{x^{2}}\left(\frac{x}{a}\right)^{2 b} .
\end{aligned}
$$

$R_{i}^{i}$

$$
R=6 \frac{b(2 b-1)}{x^{2}}
$$


$T_{i j}$

$$
\begin{aligned}
& \kappa T_{00}=\frac{b(2-3 b)}{x^{2}}\left(\frac{x}{a}\right)^{2 b}, \\
& \kappa T_{11}=3\left(\frac{b}{x}\right)^{2}, \\
& \kappa T_{22}=\kappa T_{33}=\frac{b(-2+3 b)}{x^{2}}\left(\frac{x}{a}\right)^{2 b} .
\end{aligned}
$$

$T^{i}{ }_{i}$

$$
T=-6 \frac{b(2 b-1)}{x^{2}} .
$$

Metric (180) is a gravitational wave solution $\left(R_{i j} \rightarrow 0\right.$ as $x \rightarrow \infty)$ provided $b<1$ and $b \neq 0$.

Case 7.

$$
\begin{aligned}
& d s^{2}=d t^{2}-\left(\frac{t}{a}\right)^{2 b}\left(d x^{2}+d y^{2}+d z^{2}\right) \\
&(b \neq 0,1, a \neq 0) .
\end{aligned}
$$

Metric (187) is a gravitational wave solution when $R_{i j} \rightarrow 0$ as $t \rightarrow \infty$ provided $b<1$ and $b \neq 0$.

Case 8.

$$
\begin{aligned}
& \text { (i) } d s^{2}=d t^{2}-d x^{2}-e^{\mu(t \pm x)}\left(d y^{2}+d z^{2}\right), \\
& \text { (ii) } d s^{2}=d t^{2}-d x^{2}-\left(\frac{t}{a} \pm \frac{x}{b}\right)^{2}\left(d y^{2}+d z^{2}\right),
\end{aligned}
$$

$$
(a \neq b \neq 0) .
$$

$C_{i j}^{k}$

$$
\begin{aligned}
& C_{13}^{2}=C_{\widehat{0} \hat{1}}^{0}=C_{\widehat{0} \hat{2}}^{\widehat{3}}=C_{\widehat{0} \widehat{3}}^{\hat{0}}=C_{0 \widehat{2}}^{\hat{1}}=1, \\
& C_{12}^{3}=C_{\widehat{2} \hat{3}}^{\hat{2}}=-1, \\
& C_{\hat{1} \widehat{3}}^{\hat{1}}=-\frac{1}{2}, \\
& C_{0 \hat{3}}^{0}=\frac{1}{2} .
\end{aligned}
$$

$$
R_{i j k l}
$$$$
R_{0101}=R_{0202}=R_{0303}=\frac{b(b-1)}{t^{2}}\left(\frac{t}{a}\right)^{2 b},
$$$$
R_{1212}=R_{1313}=R_{2323}=-\left(\frac{b}{t}\right)^{2}\left(\frac{t}{a}\right)^{4 b} .
$$

$$
R_{i j}
$$

$$
\begin{aligned}
& R_{00}=3 \frac{b(1-b)}{t^{2}} \\
& R_{11}=\frac{b(3 b-1)}{t^{2}}\left(\frac{t}{a}\right)^{2 b}, \\
& R_{22}=R_{33}=\frac{b(3 b-1)}{t^{2}}\left(\frac{t}{a}\right)^{2 b} .
\end{aligned}
$$

$$
R_{i}^{i}
$$

$$
R=-6 \frac{b(2 b-1)}{t^{2}}
$$

$T_{i j}$

$$
\begin{aligned}
& \kappa T_{00}=3\left(\frac{b}{t}\right)^{2}, \\
& \kappa T_{11}=\frac{b(2-3 b)}{t^{2}}\left(\frac{t}{a}\right)^{2 b}, \\
& \kappa T_{22}=\kappa T_{33}=\frac{b(2-3 b)}{t^{2}}\left(\frac{t}{a}\right)^{2 b} .
\end{aligned}
$$

$R_{i j k l}$

For (i)

$$
\begin{aligned}
& R_{0202}=R_{0303}=R_{1212}=R_{1313}=e^{2 \mu(t \pm x)}\left(\ddot{\mu}+\dot{\mu}^{2}\right), \\
& R_{0212}=R_{0313}=e^{2 \mu(t \pm x)} \pm\left(\ddot{\mu}+\dot{\mu}^{2}\right) .
\end{aligned}
$$

For (ii)

$$
R_{2323}=\frac{a^{2}-b^{2}}{\left(a^{4} b^{4}\right)}(b t \pm a x)^{2} .
$$

$R_{i j}$

For (i)

$$
\begin{aligned}
& R_{00}=R_{11}=-2\left(\ddot{\mu}+\dot{\mu}^{2}\right), \\
& R_{01}=\mp 2\left(\ddot{\mu}+\dot{\mu}^{2}\right) .
\end{aligned}
$$

For (ii)

$$
R_{22}=R_{33}=\frac{1}{a^{2}} .
$$

$R_{i}^{i}$

For (i)

$$
R=0 .
$$


TABLE 29: Conserved quantities corresponding to each Noether symmetry.

\begin{tabular}{ll}
\hline $\begin{array}{l}\text { Generator; gauge term: } \\
A\left(s, q^{i}\right)\end{array}$ & Conserved quantity: $\varphi\left(s, q^{i}, \dot{q}^{i}\right)$ \\
\hline $\mathbf{X}_{0}=\partial_{t}$ & $-2 e^{2 v(x)} \dot{t}$ \\
$\mathbf{Y}_{1}=s \partial_{y} ; A_{1}=-2 y$ & $2(y-s \dot{y})$ \\
$\mathbf{Y}_{2}=s \partial_{z} ; A_{2}=-2 z$ & $2(z-s \dot{z})$ \\
\hline
\end{tabular}

TABLE 30: Conserved quantities corresponding to each Noether symmetry.

\begin{tabular}{ll}
\hline $\begin{array}{l}\text { Generator; gauge term: } \\
A\left(s, q^{i}\right)\end{array}$ & Conserved quantity: $\varphi\left(s, q^{i}, \dot{q}^{i}\right)$ \\
\hline $\mathbf{X}_{0}=\partial_{x}$ & $2 e^{2 \lambda(t)} \dot{x}$ \\
$\mathbf{Y}_{1}=s \partial_{y} ; A_{1}=-2 y$ & $2(y-s \dot{y})$ \\
$\mathbf{Y}_{2}=s \partial_{z} ; A_{2}=-2 z$ & $2(z-s \dot{z})$ \\
\hline
\end{tabular}

For (ii)

$$
R=2\left(\frac{b}{b t \pm a x}\right)^{2}
$$

$T_{i j}$

For (i)

$$
\kappa T_{00}=\kappa T_{11}=-2\left(\ddot{\mu}+\dot{\mu}^{2}\right)^{2} .
$$

For (ii)

$$
\begin{aligned}
& \kappa T_{00}=\frac{b^{2}-a^{2}}{(b t \pm a x)^{2}}, \\
& \kappa T_{11}=\frac{a^{2}-b^{2}}{(b t \pm a x)^{2}} .
\end{aligned}
$$

$T_{i}^{i}$

For (i)

$$
T=0 .
$$

For (ii)

$$
T=-2\left(\frac{b}{b t \pm a x}\right)^{2}
$$

4.5. Seven Noether Symmetries. The Noether symmetries admitted by the metrics are presented in Tables 29-36, respectively, along with the minimal set of symmetries which is given in (7). For each metric, we have calculated the structure constants of the corresponding Lie algebra and the nonzero components of the relevant tensors.

Case 1.

$$
\begin{array}{r}
d s^{2}=e^{2 v(x)} d t^{2}-d x^{2}-d y^{2}-d z^{2} \\
\quad(v(x)=\text { arbitrary }) .
\end{array}
$$

TABLE 31: Conserved quantities corresponding to each Noether symmetry.

\begin{tabular}{ll}
\hline Generator & Conserved quantity: $\varphi\left(s, q^{i}, \dot{q}^{i}\right)$ \\
\hline $\mathbf{X}_{0}=\partial_{t}$ & $-2\left(\frac{x}{a}\right)^{2} \dot{t}$ \\
$\mathbf{X}_{4}=y \partial_{t}+\left(\frac{K}{a}\right)^{2} t \partial_{y}$ & $-2\left(\frac{x}{a}\right)^{2}(y \dot{t}-t \dot{y})$ \\
$\mathbf{X}_{5}=z \partial_{t}+\left(\frac{K}{a}\right)^{2} t \partial_{z}$ & $2\left(\frac{x}{a}\right)^{2}(z \dot{t}-t \dot{z})$ \\
\hline
\end{tabular}

$C_{i j}^{k}$

$$
\begin{aligned}
& C_{13}^{2}=C_{\widehat{01}}^{3}=C_{\widehat{02}}^{2}=1, \\
& C_{12}^{3}=-1 .
\end{aligned}
$$

$R_{i j k l}$

$$
R_{0101}=-e^{2 v}\left(v^{\prime \prime}+v^{\prime 2}\right)
$$

$R_{i j}$

$$
\begin{aligned}
& R_{00}=e^{2 v}\left(v^{\prime \prime}+v^{\prime 2}\right), \\
& R_{11}=-\left(v^{\prime \prime}+v^{\prime 2}\right) .
\end{aligned}
$$

$R_{i}^{i}$

$$
R=2\left(v^{\prime \prime}+v^{\prime 2}\right)
$$

$T_{i j}$

$$
\kappa T_{22}=\kappa T_{33}=v^{\prime \prime}+\nu^{\prime 2} .
$$

$T_{i}^{i}$

$$
T=-2\left(\nu^{\prime \prime}+v^{\prime 2}\right)
$$

Case 2.

$$
\begin{aligned}
& d s^{2}=d t^{2}-e^{2 \lambda(t)} d x^{2}-d y^{2}-d z^{2} \\
&(\lambda(t)=\text { arbitrary }) .
\end{aligned}
$$

The structure constants of the corresponding Lie algebra are the same as for (205).

$$
\begin{array}{ll}
R_{i j k l} & \\
& R_{0101}=e^{2 \lambda}\left(\ddot{\lambda}+\dot{\lambda}^{2}\right) . \\
R_{i j} & \\
R_{00} & =-\left(\ddot{\lambda}+\dot{\lambda}^{2}\right), \\
& R_{11}=e^{2 \lambda}\left(\ddot{\lambda}+\dot{\lambda}^{2}\right) .
\end{array}
$$


TABLE 32: Conserved quantities corresponding to each Noether symmetry.

Generator; gauge term: $A\left(s, q^{i}\right)$

Conserved quantity: $\varphi\left(s, q^{i}, \dot{q}^{i}\right)$

$\mathbf{X}_{0}=\partial_{t}$

$\mathbf{Y}_{1}=s \partial_{t} ; A_{1}=2 t$

$\mathbf{Y}_{2}=s \partial_{s}+\frac{1}{2} t \partial_{t}+\frac{1}{2 b+2}\left(x \partial_{x}+b y \partial_{y}+b z \partial_{z}\right)$

$-2 \dot{t}$

$2(t-s \dot{t})$

$s L+\frac{1}{b+1}\left[\left(\frac{x}{a}\right)^{2 b} x \dot{x}+b\left(\frac{x}{K}\right)^{2}(y \dot{y}+z \dot{z})\right]$
TABLE 33: Conserved quantities corresponding to each Noether symmetry.

\begin{tabular}{ll}
\hline Generator & Conserved quantity: $\varphi\left(s, q^{i}, \dot{q}^{i}\right)$ \\
\hline $\mathbf{X}_{0}=\partial_{t}$ & $-2 e^{2 v(x)} \dot{t}$ \\
$\mathbf{X}_{4}=y \partial_{t}+t \partial_{y}$ & $2 e^{2 v(x)}(y \dot{t}-t \dot{y})$ \\
$\mathbf{X}_{5}=z \partial_{t}+t \partial_{z}$ & $2 e^{2 v(x)}(z \dot{t}-t \dot{z})$ \\
\hline
\end{tabular}

TABLE 34: Conserved quantities corresponding to each Noether symmetry.

\begin{tabular}{ll}
\hline Generator & Conserved quantity: $\varphi\left(s, q^{i}, \dot{q}^{i}\right)$ \\
\hline $\mathbf{X}_{0}=\partial_{x}$ & $2 e^{2 \lambda(t)} \dot{x}$ \\
$\mathbf{X}_{4}=y \partial_{x}-x \partial_{y}$ & $2 e^{2 \lambda(t)}(y \dot{x}-x \dot{y})$ \\
$\mathbf{X}_{5}=z \partial_{x}-x \partial_{z}$ & $2 e^{2 \lambda(t)}(z \dot{x}-x \dot{z})$ \\
\hline
\end{tabular}

$R_{i}^{i}$

$$
R=-2\left(\ddot{\lambda}+\dot{\lambda}^{2}\right) \text {. }
$$

$T_{i j}$

$$
\kappa T_{22}=\kappa T_{33}=-\left(\ddot{\lambda}+\dot{\lambda}^{2}\right) .
$$

$T_{i}^{i}$

$$
T=2\left(\ddot{\lambda}+\dot{\lambda}^{2}\right)
$$

Case 3.

$$
\begin{aligned}
d s^{2}=\left(\frac{x}{a}\right)^{2} d t^{2}-e^{2 \lambda(x)} d x^{2}-\left(\frac{x}{K}\right)^{2}\left(d y^{2}+d z^{2}\right), & (a \neq K \neq 0, \lambda(x)=\text { arbitrary }) \\
C_{i j}^{k} & \\
C_{13}^{2}= & C_{34}^{0}=C_{25}^{0}=C_{14}^{5}=1, \\
C_{12}^{3}= & C_{15}^{4}=-1, \\
C_{45}^{1} & =-\left(\frac{K}{a}\right)^{2}, \\
C_{04}^{3} & =C_{05}^{2}=\left(\frac{K}{a}\right)^{2} .
\end{aligned}
$$$$
(a \neq K \neq 0, \lambda(x)=\text { arbitrary }) \text {. }
$$

$R_{i j k l}$

$$
\begin{aligned}
& R_{0101}=\frac{\lambda^{\prime} x}{a^{2}}, \\
& R_{0202}=R_{0303}=-\frac{1}{K^{2}}\left(\frac{x}{a}\right)^{2} e^{-2 \lambda}, \\
& R_{1212}=R_{1313}=-\frac{\lambda^{\prime} x}{K^{2}}, \\
& R_{2323}=\left(\frac{x}{K^{2}}\right)^{2} e^{-2 \lambda} .
\end{aligned}
$$

$$
\begin{aligned}
& R_{00}=\left(\frac{2-\lambda^{\prime} x}{a^{2}}\right) e^{-2 \lambda}, \\
& R_{11}=\frac{3 \lambda^{\prime}}{x} \\
& R_{22}=R_{33}=-\left(\frac{2-\lambda^{\prime} x}{K^{2}}\right) e^{-2 \lambda}
\end{aligned}
$$

$R_{i}^{i}$

$$
R=6\left(\frac{1-\lambda^{\prime} x}{x^{2}}\right) e^{-2 \lambda}
$$

$T_{i j}$

$$
\begin{aligned}
& \kappa T_{00}=\left(\frac{-1+2 \lambda^{\prime} x}{a^{2}}\right) e^{-2 \lambda}, \\
& \kappa T_{11}=\frac{3}{x^{2}}, \\
& \kappa T_{22}=\kappa T_{33}=\left(\frac{1-2 \lambda^{\prime} x}{K^{2}}\right) e^{-2 \lambda} .
\end{aligned}
$$

$T_{i}^{i}$

$$
T=-6\left(\frac{1-\lambda^{\prime} x}{x^{2}}\right) e^{-2 \lambda}
$$

Case 4.

$$
\begin{array}{r}
d s^{2}=d t^{2}-\left(\frac{x}{a}\right)^{2 b} d x^{2}-\left(\frac{x}{K}\right)^{2}\left(d y^{2}+d z^{2}\right), \\
(a \neq K \neq 0, \quad b \neq 0) .
\end{array}
$$


TABLE 35: Conserved quantities corresponding to each Noether symmetry.

\begin{tabular}{ll}
\hline Generator & Conserved quantity: $\varphi\left(s, q^{i}, \dot{q}^{i}\right)$ \\
\hline $\mathbf{X}_{0}=-a \partial_{t}+y \partial_{y}+z \partial_{z}$ & $2\left[a e^{2 v(x)} \dot{t}+e^{2(t / a+v(x))}(y \dot{y}+z \dot{z})\right]$ \\
$\mathbf{X}_{4}=-\frac{2 y}{a} \partial_{t}+\left(e^{-2 t / a}+\frac{1}{a^{2}}\left(y^{2}-z^{2}\right)\right) \partial_{y}+\frac{2}{a^{2}} y z \partial_{z}$ & $2\left[\frac{2 y}{a} e^{2 v(x)} \dot{t}+e^{2(t / a+v(x))}\left\{\left(e^{-2 t / a}+\frac{1}{a^{2}}\left(y^{2}-z^{2}\right)\right) \dot{y}+\frac{2 y}{a^{2}} z \dot{z}\right\}\right]$ \\
$\mathbf{X}_{5}=-a z \partial_{t}+z y \partial_{y}+\frac{1}{2}\left(a^{2} e^{-2 t / a}-y^{2}+z^{2}\right) \partial_{z}$ & $2\left[a z e^{2 v(x)} \dot{t}+e^{2(t / a+v(x))}\left\{z y \dot{y}-\frac{1}{2}\left(a^{2} e^{-2 t / a}-y^{2}+z^{2}\right) \dot{z}\right\}\right]$ \\
\hline
\end{tabular}

TABLE 36: Conserved quantities corresponding to each Noether symmetry.

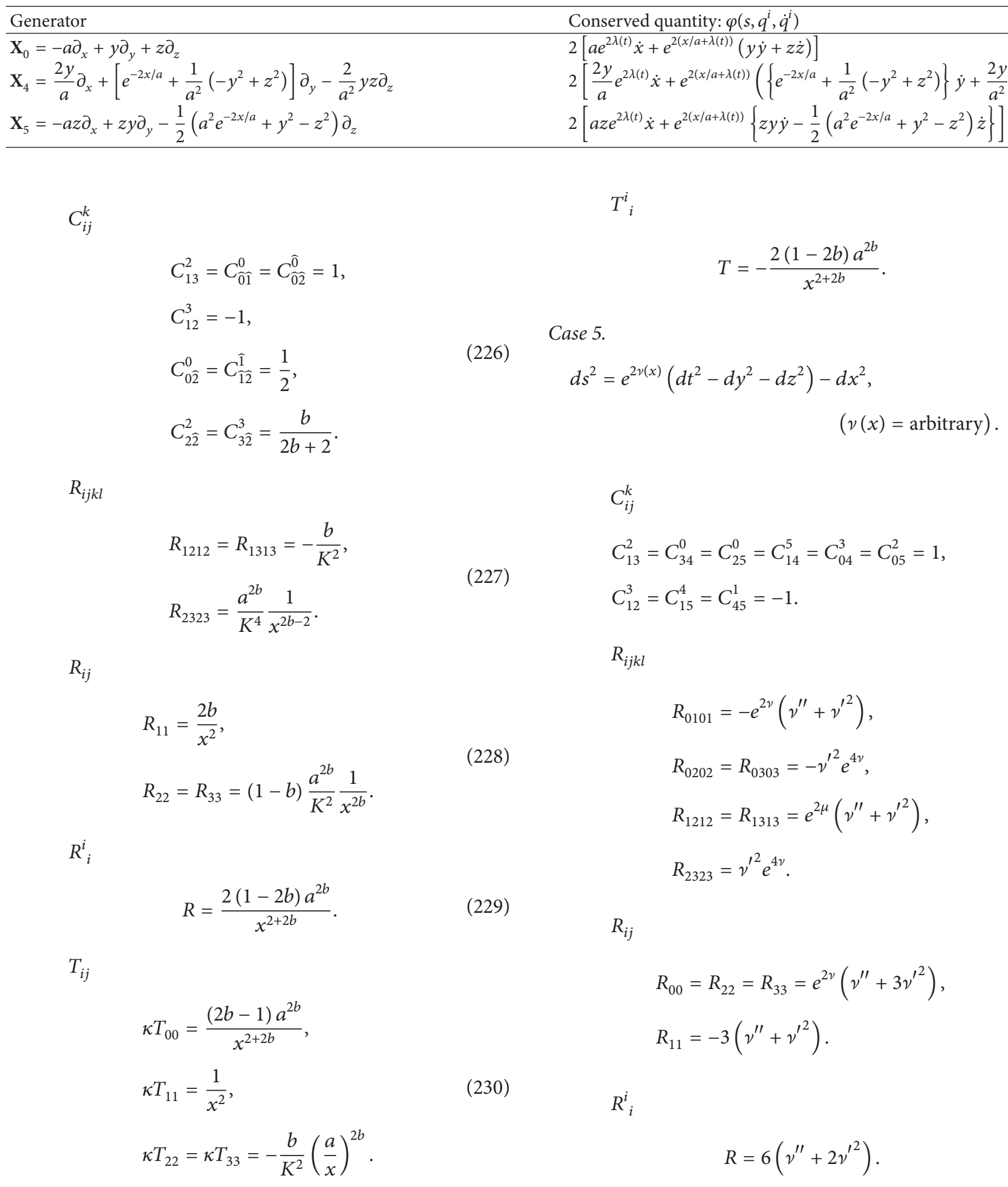


$T_{i j}$

$$
\begin{aligned}
& \kappa T_{00}=-e^{2 v}\left(2 v^{\prime \prime}+3 v^{\prime 2}\right), \\
& \kappa T_{11}=3 v^{\prime 2}, \\
& \kappa T_{22}=\kappa T_{33}=e^{2 v}\left(2 v^{\prime \prime}+3 v^{\prime 2}\right) .
\end{aligned}
$$

$T_{i}^{i}$

$$
T=-6\left(v^{\prime \prime}+2 v^{\prime 2}\right) .
$$

Case 6.

$$
\begin{array}{r}
d s^{2}=d t^{2}-e^{2 \lambda(t)}\left(d x^{2}+d y^{2}+d z^{2}\right) \\
(\lambda(t)=\text { arbitrary }) .
\end{array}
$$

$C_{i j}^{k}$

$$
\begin{aligned}
& C_{13}^{2}=C_{34}^{0}=C_{25}^{0}=C_{14}^{5}=C_{45}^{1}=1, \\
& C_{12}^{3}=C_{04}^{3}=C_{05}^{2}=C_{15}^{4}=-1 .
\end{aligned}
$$

$R_{i j k l}$

$$
\begin{aligned}
& R_{0101}=R_{0202}=R_{0303}=e^{2 \lambda}\left(\ddot{\lambda}+\dot{\lambda}^{2}\right), \\
& R_{1212}=R_{1313}=R_{2323}=-\dot{\lambda}^{2} e^{4 \lambda} .
\end{aligned}
$$

$$
R_{i}^{i}
$$

$$
\begin{aligned}
& R_{00}=-3\left(\ddot{\lambda}+\dot{\lambda}^{2}\right), \\
& R_{11}=R_{22}=R_{33}=e^{2 \lambda}\left(\ddot{\lambda}+3 \dot{\lambda}^{2}\right) .
\end{aligned}
$$

$$
R=-6\left(\ddot{\lambda}+2 \dot{\lambda}^{2}\right) .
$$

$T_{i j}$

$$
\begin{aligned}
& \kappa T_{00}=3 \dot{\lambda}^{2}, \\
& \kappa T_{11}=\kappa T_{22}=\kappa T_{33}=-e^{2 \lambda}\left(2 \ddot{\lambda}+3 \dot{\lambda}^{2}\right) .
\end{aligned}
$$$$
T^{i}{ }_{i}
$$

$$
T=6\left(\ddot{\lambda}+2 \dot{\lambda}^{2}\right) .
$$

Case 7.

(i) $d s^{2}$

$$
=e^{2 \gamma(x)} d t^{2}-d x^{2}-e^{2(t / a+\gamma(x))}\left(d y^{2}+d z^{2}\right),
$$

(ii) $d s^{2}$

$$
\begin{aligned}
= & \sinh ^{2}\left(\frac{x}{b}\right) d t^{2}-d x^{2} \\
& -e^{2 t / a} \sinh ^{2}\left(\frac{x}{b}\right)\left(d y^{2}+d z^{2}\right),
\end{aligned}
$$

(iii) $d s^{2}$

$$
\begin{aligned}
= & \cosh ^{2}\left(\frac{x}{b}\right) d t^{2}-d x^{2} \\
& -e^{2 t / a} \cosh ^{2}\left(\frac{x}{b}\right)\left(d y^{2}+d z^{2}\right),
\end{aligned}
$$

where $a \neq 0 \neq b$ and $\nu(x)$ is an arbitrary function.

$$
C_{i j}^{k}
$$

$$
\begin{aligned}
& C_{13}^{2}=C_{04}^{4}=C_{05}^{5}=C_{35}^{1}=C_{25}^{0}=1, \\
& C_{12}^{3}=-1, \\
& C_{14}^{5}=C_{34}^{0}=\frac{2}{a^{2}}, \\
& C_{15}^{4}=C_{24}^{1}=-\frac{a^{2}}{2} .
\end{aligned}
$$

$R_{i j k l}$

$$
\text { For (i) }
$$

$$
\begin{aligned}
& R_{0101}=-e^{2 \nu}\left(\nu^{\prime \prime}+\nu^{\prime 2}\right), \\
& R_{0202}=R_{0303}=e^{2(t / a+v(x))}\left(\frac{1}{a^{2}}-\nu^{\prime 2} e^{2 v}\right), \\
& R_{1212}=R_{1313}=e^{2 \nu}\left(\nu^{\prime \prime}+\nu^{\prime 2}\right), \\
& R_{2323}=\nu^{\prime 2} e^{4(t / a+v(x))} .
\end{aligned}
$$

For (ii)

$$
\begin{aligned}
R_{0101} & =-\frac{1}{b^{2}} \sinh ^{2}\left(\frac{x}{b}\right), \\
R_{0202} & =R_{0303} \\
& =e^{2 t / a} \sinh ^{2}\left(\frac{x}{b}\right)\left[\frac{1}{a^{2}}-\frac{1}{b^{2}} \cosh ^{2}\left(\frac{x}{b}\right)\right], \\
R_{1212} & =R_{1313}=\frac{1}{b^{2}} e^{2 t / a} \sinh ^{2}\left(\frac{x}{b}\right), \\
R_{2323} & =e^{4 t / a} \sinh ^{2}\left(\frac{x}{b}\right)\left[-\frac{1}{a^{2}}+\frac{1}{b^{2}} \cosh ^{2}\left(\frac{x}{b}\right)\right] .
\end{aligned}
$$


For (iii)

$$
\begin{aligned}
R_{0101} & =-\frac{1}{b^{2}} \cosh ^{2}\left(\frac{x}{b}\right) \\
R_{0202} & =R_{0303} \\
& =e^{2 t / a} \cosh ^{2}\left(\frac{x}{b}\right)\left[\frac{1}{a^{2}}-\frac{1}{b^{2}} \sinh ^{2}\left(\frac{x}{b}\right)\right], \\
R_{1212} & =R_{1313}=\frac{1}{b^{2}} e^{2 t / a} \cosh ^{2}\left(\frac{x}{b}\right) \\
R_{2323} & =e^{4 t / a} \cosh ^{2}\left(\frac{x}{b}\right)\left[-\frac{1}{a^{2}}+\frac{1}{b^{2}} \sinh ^{2}\left(\frac{x}{b}\right)\right] . \\
R_{i j} &
\end{aligned}
$$

For (i)

$$
\begin{aligned}
& R_{00}=e^{2 v}\left(v^{\prime \prime}+3 v^{\prime 2}\right), \\
& R_{11}=-3\left(v^{\prime \prime}+v^{\prime 2}\right), \\
& R_{22}=R_{33}=e^{2(t / a+v(x))}\left(v^{\prime \prime}+3 v^{\prime 2}\right) .
\end{aligned}
$$$$
\text { For (ii) }
$$

$$
\begin{aligned}
R_{00} & =\frac{1}{b^{2}}\left[\sinh ^{2}\left(\frac{x}{b}\right)+2 \cosh ^{2}\left(\frac{x}{b}\right)\right]-\frac{2}{a^{2}}, \\
R_{11} & =-\frac{3}{b^{2}} \\
R_{22} & =R_{33} \\
& =e^{2 t / a}\left[\frac{2}{a^{2}}-\frac{1}{b^{2}}\left\{\sinh ^{2}\left(\frac{x}{b}\right)+2 \cosh ^{2}\left(\frac{x}{b}\right)\right\}\right] .
\end{aligned}
$$$$
\text { For (iii) }
$$

$$
\begin{aligned}
R_{00}= & \frac{1}{b^{2}}\left[2 \sinh ^{2}\left(\frac{x}{b}\right)+\cosh ^{2}\left(\frac{x}{b}\right)\right]-\frac{2}{a^{2}}, \\
R_{11} & =-\frac{3}{b^{2}} \\
R_{22} & =R_{33} \\
= & e^{2 t / a}\left[\frac{2}{a^{2}}-\frac{1}{b^{2}}\left\{2 \sinh ^{2}\left(\frac{x}{b}\right)+\cosh ^{2}\left(\frac{x}{b}\right)\right\}\right] . \\
& R_{i}^{i}
\end{aligned}
$$

For (i)

$$
R=6\left(v^{\prime \prime}+2 v^{\prime 2}\right)-\frac{6}{a^{2}} e^{-2 v} .
$$

For (ii)

$$
R=\frac{6}{b^{2}}\left[1+2 \operatorname{coth}^{2}\left(\frac{x}{b}\right)\right]-\frac{6}{a^{2}} \operatorname{csch}^{2}\left(\frac{x}{b}\right) .
$$

For (iii)

$$
\begin{aligned}
& R=\frac{6}{b^{2}}\left[1+2 \tanh ^{2}\left(\frac{x}{b}\right)\right]-\frac{6}{a^{2}} \operatorname{sech}^{2}\left(\frac{x}{b}\right) . \\
& T_{i j}
\end{aligned}
$$$$
\text { For (i) }
$$

$$
\begin{aligned}
& \kappa T_{00}=-e^{2 v}\left(2 \nu^{\prime \prime}+3 v^{\prime 2}\right)+\frac{1}{a^{2}}, \\
& \kappa T_{11}=3\left(-\frac{1}{a^{2}} e^{-2 v}+v^{\prime 2}\right), \\
& \kappa T_{22}=\kappa T_{33}=e^{2 t / a}\left(-\frac{1}{a^{2}}+e^{2 v}\left(2 \nu^{\prime \prime}+3 v^{\prime 2}\right)\right) .
\end{aligned}
$$

For (ii)

For (i)

$$
T=-6\left(\nu^{\prime \prime}+2 \nu^{\prime 2}\right)+\frac{6}{a^{2}} e^{-2 \nu}
$$

$$
\kappa T_{22}=\kappa T_{33}
$$

$$
=e^{2 t / a}\left[\frac{2}{b^{2}} \sinh ^{2}\left(\frac{x}{b}\right)+\frac{1}{b^{2}} \cosh ^{2}\left(\frac{x}{b}\right)-\frac{1}{a^{2}}\right] .
$$

For (iii)

$$
\begin{aligned}
& \kappa T_{00}=\frac{1}{a^{2}}-\frac{1}{b^{2}}\left[\sinh ^{2}\left(\frac{x}{b}\right)+2 \cosh ^{2}\left(\frac{x}{b}\right)\right], \\
& \kappa T_{11}=3\left[\frac{1}{b^{2}} \tanh ^{2}\left(\frac{x}{b}\right)-\frac{1}{a^{2}} \operatorname{sech}^{2}\left(\frac{x}{b}\right)\right], \\
& \kappa T_{22}=\kappa T_{33} \\
&=e^{2 t / a}\left[\frac{2}{b^{2}} \cosh ^{2}\left(\frac{x}{b}\right)+\frac{1}{b^{2}} \sinh ^{2}\left(\frac{x}{b}\right)-\frac{1}{a^{2}}\right] . \\
& T_{i}^{i}
\end{aligned}
$$$$
\text { For (ii) }
$$$$
T=-\frac{6}{b^{2}}\left[1+2 \operatorname{coth}^{2}\left(\frac{x}{b}\right)\right]-\frac{6}{a^{2}} \operatorname{csch}^{2}\left(\frac{x}{b}\right) .
$$

For (iii)

$$
T=-\frac{6}{b^{2}}\left[1+2 \tanh ^{2}\left(\frac{x}{b}\right)\right]+\frac{6}{a^{2}} \operatorname{sech}^{2}\left(\frac{x}{b}\right) .
$$


Case 8.

(i) $d s^{2}$

$$
=d t^{2}-e^{2 \lambda(t)} d x^{2}-e^{2(x / a+\lambda(t))}\left(d y^{2}+d z^{2}\right),
$$

(ii) $d s^{2}$

$$
\begin{aligned}
= & d t^{2}-\sinh ^{2}\left(\frac{t}{b}\right) d x^{2} \\
& -e^{2 x / a} \sinh ^{2}\left(\frac{t}{b}\right)\left(d y^{2}+d z^{2}\right),
\end{aligned}
$$

(iii) $d s^{2}$

$$
\begin{aligned}
= & d t^{2}-\cosh ^{2}\left(\frac{t}{b}\right) d x^{2} \\
& -e^{2 x / a} \cosh ^{2}\left(\frac{t}{b}\right)\left(d y^{2}+d z^{2}\right),
\end{aligned}
$$

where $a \neq 0 \neq b$ and $\lambda(t)$ is an arbitrary function.

$$
\begin{array}{ll}
C_{i j}^{k} & \\
C_{13}^{2} & =C_{04}^{4}=C_{05}^{5}=C_{35}^{1}=C_{25}^{0}=1, \\
C_{12}^{3} & =-1, \\
C_{14}^{5} & =C_{34}^{0}=-\frac{2}{a^{2}}, \\
C_{15}^{4} & =C_{24}^{1}=\frac{a^{2}}{2} . \\
R_{i j k l} &
\end{array}
$$

For (i)

$$
\begin{aligned}
& R_{0101}=e^{2 \lambda}\left(\ddot{\lambda}+\dot{\lambda}^{2}\right), \\
& R_{0202}=R_{0303}=\left(\ddot{\lambda}+\dot{\lambda}^{2}\right) e^{2(x / a+\lambda)}, \\
& R_{1212}=R_{1313}=\left(\frac{1}{a^{2}}-\dot{\lambda}^{2} e^{2 \lambda}\right) e^{2(\lambda+x / a)}, \\
& R_{2323}=\left(\frac{1}{a^{2}} e^{-2 \lambda}-\dot{\lambda}^{2}\right) e^{4(\lambda+x / a)} .
\end{aligned}
$$$$
\text { For (ii) }
$$$$
R_{0101}=\frac{1}{b^{2}} \sinh ^{2}\left(\frac{t}{b}\right) \text {, }
$$$$
R_{0202}=R_{0303}=\frac{1}{b^{2}} e^{2 x / a} \sinh ^{2}\left(\frac{t}{b}\right),
$$$$
R_{1212}=R_{1313}
$$$$
=e^{2 x / a} \sinh ^{2}\left(\frac{t}{b}\right)\left[\frac{1}{a^{2}}-\frac{1}{b^{2}} \cosh ^{2}\left(\frac{t}{b}\right)\right],
$$$$
R_{2323}=e^{4 x / a} \sinh ^{2}\left(\frac{t}{b}\right)\left[\frac{1}{a^{2}}-\frac{1}{b^{2}} \cosh ^{2}\left(\frac{t}{b}\right)\right] \text {. }
$$

For (iii)

$$
\begin{aligned}
R_{0101} & =\frac{1}{b^{2}} \cosh ^{2}\left(\frac{t}{b}\right), \\
R_{0202} & =R_{0303}=\frac{1}{b^{2}} e^{2 x / a} \cosh ^{2}\left(\frac{t}{b}\right), \\
R_{1212} & =R_{1313} \\
& =e^{2 x / a} \cosh ^{2}\left(\frac{t}{b}\right)\left(\frac{1}{a^{2}}-\frac{1}{b^{2}} \sinh ^{2}\left(\frac{t}{b}\right)\right), \\
R_{2323} & =e^{4 x / a} \cosh ^{2}\left(\frac{t}{b}\right)\left(\frac{1}{a^{2}}-\frac{1}{b^{2}} \sinh ^{2}\left(\frac{t}{b}\right)\right) .
\end{aligned}
$$

$R_{i j}$

$$
\text { For (i) }
$$

$$
\begin{aligned}
& R_{00}=-3\left(\ddot{\lambda}+\dot{\lambda}^{2}\right), \\
& R_{11}=e^{2 \lambda}\left(\ddot{\lambda}+3 \dot{\lambda}^{2}\right),
\end{aligned}
$$

$$
R_{22}=R_{33}=-e^{2 x / a}\left[e^{2 \lambda}\left(\ddot{\lambda}+\dot{\lambda}^{2}\right)-\frac{2}{a^{2}}\right] .
$$

For (ii)

$$
\begin{aligned}
& R_{00}=-\frac{3}{b^{2}} \\
& R_{11}=\frac{1}{b^{2}}\left[\sinh ^{2}\left(\frac{t}{b}\right)+2 \cosh ^{2}\left(\frac{t}{b}\right)\right]-\frac{2}{a^{2}}, \\
& R_{22}=R_{33} \\
& =e^{2 x / a}\left[-\frac{2}{a^{2}} \sinh ^{2}\left(\frac{t}{b}\right)+\frac{1}{b^{2}}\left\{1+2 \operatorname{coth}^{2}\left(\frac{t}{b}\right)\right\}\right] .
\end{aligned}
$$

For (iii)

$$
\begin{aligned}
R_{00} & =-\frac{3}{b^{2}} \\
R_{11} & =\frac{1}{b^{2}}\left[\cosh ^{2}\left(\frac{t}{b}\right)+2 \sinh ^{2}\left(\frac{t}{b}\right)\right]-\frac{2}{a^{2}}, \\
R_{22} & =R_{33} \\
& =e^{2 x / a}\left[\frac{2}{b^{2}} \sinh ^{2}\left(\frac{t}{b}\right)+\frac{1}{b^{2}} \cosh ^{2}\left(\frac{t}{b}\right)-\frac{1}{a^{2}}\right] . \\
& R_{i}^{i}
\end{aligned}
$$$$
\text { For (i) }
$$$$
R=\frac{6}{a^{2}} e^{-2 \lambda}-6\left(\ddot{\lambda}+2 \dot{\lambda}^{2}\right) .
$$

For (ii)

$$
R=\frac{6}{a^{2}} \operatorname{csch}^{2}\left(\frac{t}{b}\right)-\frac{6}{b^{2}}\left[1+2 \operatorname{coth}^{2}\left(\frac{t}{b}\right)\right] .
$$


For (iii)

$$
R=\frac{6}{a^{2}} \operatorname{sech}^{2}\left(\frac{t}{b}\right)-\frac{6}{b^{2}}\left[1+\tanh ^{2}\left(\frac{t}{b}\right)\right] .
$$

$T_{i j}$

For (i)

$$
\begin{aligned}
& \kappa T_{00}=3\left(-\frac{1}{a^{2}} e^{-2 \lambda}+\dot{\lambda}^{2}\right) \\
& \kappa T_{11}=\frac{1}{a^{2}}-\left(2 \ddot{\lambda}+3 \dot{\lambda}^{2}\right) e^{2 \lambda}, \\
& \kappa T_{22}=\kappa T_{33}=\left[\frac{1}{a^{2}} e^{-2 \lambda}-\left(2 \ddot{\lambda}+3 \dot{\lambda}^{2}\right)\right] e^{2(\lambda+x / a)} .
\end{aligned}
$$

For (ii)

$$
\begin{aligned}
\kappa T_{00} & =3\left[\frac{1}{b^{2}} \operatorname{coth}^{2}\left(\frac{t}{b}\right)-\frac{1}{a^{2}} \operatorname{csch}^{2}\left(\frac{t}{b}\right)\right], \\
\kappa T_{11} & =\frac{1}{a^{2}}-\left[\sinh ^{2}\left(\frac{t}{b}\right)+\frac{1}{b^{2}} \cosh ^{2}\left(\frac{x}{b}\right)\right], \\
\kappa T_{22} & =\kappa T_{33} \\
& =e^{2 x / a}\left[\frac{1}{a^{2}}-\frac{2}{b^{2}} \sinh ^{2}\left(\frac{t}{b}\right)-\frac{1}{b^{2}} \cosh ^{2}\left(\frac{t}{b}\right)\right] .
\end{aligned}
$$

For (iii)

$$
\begin{aligned}
& \kappa T_{00}=3\left[\frac{1}{b^{2}} \tanh ^{2}\left(\frac{t}{b}\right)-\frac{1}{a^{2}} \operatorname{sech}^{2}\left(\frac{t}{b}\right)\right] \\
& \kappa T_{11}=\frac{1}{a^{2}}-\frac{1}{b^{2}}\left[\sinh ^{2}\left(\frac{t}{b}\right)+2 \cosh ^{2}\left(\frac{x}{b}\right)\right] \\
& \kappa T_{22}=\kappa T_{33} \\
&=e^{2 x / a}\left[\frac{1}{a^{2}}-\frac{2}{b^{2}} \cosh ^{2}\left(\frac{t}{b}\right)-\frac{1}{b^{2}} \sinh ^{2}\left(\frac{t}{b}\right)\right] . \\
& T_{i}^{i}
\end{aligned}
$$$$
\text { For (i) }
$$$$
T=-\frac{6}{a^{2}} e^{-2 \lambda}+6\left(\ddot{\lambda}+2 \dot{\lambda}^{2}\right)
$$

For (ii)

$$
T=-\frac{6}{a^{2}} \operatorname{csch}^{2}\left(\frac{t}{b}\right)+\frac{6}{b^{2}}\left[1+2 \operatorname{coth}^{2}\left(\frac{t}{b}\right)\right] .
$$

For (iii)

$$
T=-\frac{6}{a^{2}} \operatorname{sech}^{2}\left(\frac{t}{b}\right)+\frac{6}{b^{2}}\left[1+\tanh ^{2}\left(\frac{t}{b}\right)\right] .
$$

TABLE 37: Conserved quantities corresponding to each Noether symmetry.

\begin{tabular}{ll}
\hline Generator; gauge term: & Conserved quantity: $\varphi\left(s, q^{i}, \dot{q}^{i}\right)$ \\
$A\left(s, q^{i}\right)$ & $-2 \dot{t}$ \\
\hline $\mathbf{X}_{0}=\partial_{t}$ & $2(t-s \dot{t})$ \\
$\mathbf{Y}_{1}=s \partial_{t} ; A_{1}=2 t$ & \\
\hline
\end{tabular}

TABLE 38: Conserved quantities corresponding to each Noether symmetry.

\begin{tabular}{ll}
\hline Generator & Conserved quantity: $\varphi\left(s, q^{i}, \dot{q}^{i}\right)$ \\
\hline $\mathbf{X}_{0}=\partial_{t}$ & $-2\left(\frac{x}{a}\right)^{2 b} \dot{t}$ \\
$\mathbf{X}_{4}=-b t \partial_{t}+$ & $2\left[b\left(\frac{x}{a}\right)^{2 b} t \dot{t}+\left(\frac{c}{x}\right)^{2} x \dot{x}-\left(\frac{x}{K}\right)^{2}(y \dot{y}+z \dot{z})\right]$ \\
$x \partial_{x}-y \partial_{y}-z \partial_{z}$ & \\
\hline
\end{tabular}

4.6. Six Noether Symmetries. In this section, metrics admit six Noether symmetries. Four of them are given in (7) and the rest of the symmetries along with their conserved quantities are given in Tables 37-43. Also, the structure constants of the corresponding Lie algebra and the nonzero components of the relevant tensors are calculated for each metric.

Case 1.

$$
\begin{aligned}
d s^{2}=d t^{2}-e^{2 \lambda(x)} d x^{2}- & \left(\frac{x}{K}\right)^{2}\left(d y^{2}+d z^{2}\right) \\
& (K \neq 0, \lambda(x)=\text { arbitrary }) .
\end{aligned}
$$

$C_{i j}^{k}$

$$
\begin{aligned}
& C_{13}^{2}=C_{\widehat{01}}^{0}=1, \\
& C_{12}^{3}=-1 .
\end{aligned}
$$

$R_{i j k l}$

$$
R_{2323}=\left(\frac{x}{K^{2}}\right)^{2} e^{-2 \lambda}
$$

$R_{i j}$

$$
R_{22}=R_{33}=-\frac{1-\lambda^{\prime} x}{K^{2}} e^{-2 \lambda}
$$

$R_{i}^{i}$

$$
R=2\left(\frac{1-2 \lambda^{\prime} x}{x^{2}}\right) e^{-2 \lambda}
$$

$T_{i j}$

$$
\begin{aligned}
& \kappa T_{00}=-\frac{1-2 \lambda^{\prime} x}{x^{2}} e^{-2 \lambda}, \\
& \kappa T_{11}=\frac{1}{x^{2}} \\
& \kappa T_{22}=\kappa T_{33}=-\frac{\lambda^{\prime} x}{K^{2}} e^{-2 \lambda} .
\end{aligned}
$$


TABLE 39: Conserved quantities corresponding to each Noether symmetry.

Generator; gauge term: $A\left(s, q^{i}\right)$

$\mathbf{X}_{0}=\partial_{t}$

$\mathbf{Y}_{1}=s \partial_{s}+\frac{1}{2 d-2}\left[(b+d-1) t \partial_{t}-x \partial_{x}+d\left(y \partial_{y}+z \partial_{z}\right)\right]$
Conserved quantity: $\varphi\left(s, q^{i}, \dot{q}^{i}\right)$

$-2\left(\frac{x}{a}\right)^{2 b} \dot{t}$

$s L-\frac{1}{d-1}\left[(b+d-1)\left(\frac{x}{a}\right)^{2 b} t \dot{t}-\left(\frac{c}{x}\right)^{2 d} x \dot{x}+d\left(\frac{x}{K}\right)^{2}(y \dot{y}+z \dot{z})\right]$
TABLE 40: Conserved quantities corresponding to each Noether symmetry.

\begin{tabular}{ll}
\hline Generator & Conserved quantity: $\varphi\left(s, q^{i}, \dot{q}^{i}\right)$ \\
\hline $\mathbf{X}_{0}=\partial_{t}$ & $-2 e^{2 x / a} \dot{t}$ \\
$\mathbf{X}_{4}=\partial_{x}-\frac{1}{a} t \partial_{t}-$ & $2\left[\frac{1}{a} e^{2 x / a} t \dot{t}+\dot{x}-\frac{1}{b} e^{2 x / b}(y \dot{y}+z \dot{z})\right]$ \\
$\frac{1}{b}\left(y \partial_{y}+z \partial_{z}\right)$ & \\
\hline
\end{tabular}

TABLE 41: Conserved quantities corresponding to each Noether symmetry.

\begin{tabular}{ll}
\hline Generator & Conserved quantity: $\varphi\left(s, q^{i}, \dot{q}^{i}\right)$ \\
\hline $\mathbf{X}_{0}=\partial_{x}$ & $2 e^{2 t / b} \dot{x}$ \\
$\mathbf{X}_{4}=\partial_{t}-\frac{1}{b} x \partial_{x}-$ & $2\left[-\dot{t}+\frac{1}{b} e^{2 x / b} x \dot{x}-\frac{1}{a} e^{2 x / a}(y \dot{y}+z \dot{z})\right]$ \\
$\frac{1}{a}\left(y \partial_{y}+z \partial_{z}\right)$ & \\
\hline
\end{tabular}

TABLE 42: Conserved quantities corresponding to each Noether symmetry.

\begin{tabular}{ll}
\hline $\begin{array}{l}\text { Generator; gauge } \\
\text { term: } A\left(s, q^{i}\right)\end{array}$ & Conserved quantity: $\varphi\left(s, q^{i}, \dot{q}^{i}\right)$ \\
\hline $\mathbf{Y}_{1}=\frac{1}{2}\left(s^{2} \partial_{s}+s t \partial_{t}\right) ;$ & $\frac{1}{2} s^{2} L-s t \dot{t}+\frac{1}{2} t^{2}$ \\
$A_{1}=\frac{1}{2} t^{2}$ & $s L-t \dot{t}$ \\
$\mathbf{Y}_{2}=s \partial_{s}+\frac{1}{2} t \partial_{t}$ & \\
\hline
\end{tabular}

TABLE 43: Conserved quantities corresponding to each Noether symmetry.

\begin{tabular}{ll}
\hline $\begin{array}{l}\text { Generator; gauge } \\
\text { term: } A\left(s, q^{i}\right)\end{array}$ & Conserved quantity: $\varphi\left(s, q^{i}, \dot{q}^{i}\right)$ \\
\hline $\mathbf{Y}_{1}=\frac{1}{2}\left(s^{2} \partial_{s}+s x \partial_{x}\right) ;$ & $\frac{1}{2} s^{2} L+s x \dot{x}-\frac{1}{2} x^{2}$ \\
$A_{1}=-\frac{1}{2} x^{2}$ & \\
$\mathbf{Y}_{2}=s \partial_{s}+\frac{1}{2} x \partial_{x}$ & $s L+x \dot{x}$ \\
\hline
\end{tabular}

$T_{i}^{i}$
Case 2.

$$
\begin{aligned}
d s^{2}=\left(\frac{x}{a}\right)^{2 b} d t^{2}-\left(\frac{c}{x}\right)^{2} d x^{2}-\left(\frac{x}{K}\right)^{2}\left(d y^{2}+d z^{2}\right) & (b \neq 0,1, a \neq c \neq K \neq 0) . \\
C_{i j}^{k} & C_{13}^{2}=1, \\
C_{04}^{0} & =-b, \\
C_{12}^{3} & =C_{24}^{2}=C_{34}^{3}=-1 .
\end{aligned}
$$

$R_{i j k l}$

$$
\begin{aligned}
& R_{0101}=b\left(1+\frac{1-b}{x^{2}}\right)\left(\frac{x}{a}\right)^{2 b}, \\
& R_{0202}=R_{0303}=-\frac{b}{K^{2}}\left(\frac{x}{a}\right)^{2 b}\left(\frac{x}{c}\right)^{2}, \\
& R_{1212}=R_{1313}=-\frac{x}{K^{2}} \\
& R_{2323}=\frac{1}{c^{2}}\left(\frac{x}{K}\right)^{4} .
\end{aligned}
$$

$R_{i j}$

$$
\begin{aligned}
& R_{00}=\frac{b(1+b)}{c^{2}}\left(\frac{x}{a}\right)^{2 b}, \\
& R_{11}=2+b+\frac{b(1+b)}{x^{2}}, \\
& R_{22}=R_{33}=\frac{1+b-x^{2}}{c^{2}}\left(\frac{x}{K}\right)^{2} .
\end{aligned}
$$

$R_{i}^{i}$

$$
R=2\left(\frac{1+b+b^{2}}{x^{2}}-2-b\right)\left(\frac{x}{c}\right)^{2}
$$

$T_{i j}$

$$
\begin{aligned}
& \kappa T_{00}=\left(2-\frac{1}{x^{2}}\right)\left(\frac{x}{c}\right)^{2}\left(\frac{x}{a}\right)^{2 b}, \\
& \kappa T_{11}=(1+2 b) \frac{1}{x^{2}} \\
& \kappa T_{22}=\kappa T_{33}=-\left(1+b-\frac{b^{2}}{x^{2}}\right)\left(\frac{x}{c}\right)^{2}\left(\frac{x}{K}\right)^{2} .
\end{aligned}
$$


$T_{i}^{i}$

$$
T=-2\left(\frac{1+b+b^{2}}{x^{2}}-2-b\right)\left(\frac{x}{c}\right)^{2} .
$$

Case 3.

$$
\begin{aligned}
d s^{2}= & \left(\frac{x}{a}\right)^{2 b} d t^{2}-\left(\frac{c}{x}\right)^{2 d} d x^{2} \\
-\left(\frac{x}{K}\right)^{2}\left(d y^{2}+d z^{2}\right), & (b \neq 0,1, d \neq 0, a \neq c \neq K \neq 0) .
\end{aligned}
$$

$C_{i j}^{k}$

$$
\begin{aligned}
& C_{13}^{2}=C_{\widehat{0} \hat{1}}^{\hat{0}}=1, \\
& C_{12}^{3}=-1, \\
& C_{0 \hat{1}}^{0}=\frac{b+d-1}{2 d-2}, \\
& C_{2 \widehat{1}}^{2}=C_{3 \hat{1}}^{3}=\frac{d}{2 d-2} .
\end{aligned}
$$

$R_{i j k l}$

$$
\begin{aligned}
& R_{0101}=b\left(\frac{1-b}{x^{2}}-d\right)\left(\frac{x}{a}\right)^{2 b} \\
& R_{0202}=R_{0303}=-\frac{b}{K^{2}}\left(\frac{x}{a}\right)^{2 b}\left(\frac{x}{c}\right)^{2 d}, \\
& R_{1212}=R_{1313}=-d\left(\frac{x}{K}\right)^{2} \\
& R_{2323}=\left(\frac{x}{K^{2}}\right)^{2}\left(\frac{x}{c}\right)^{2 d}
\end{aligned}
$$

$R_{i j}$

$$
\begin{aligned}
& R_{00}=b\left(\frac{1+b}{x^{2}}-d\right)\left(\frac{x}{a}\right)^{2 b}\left(\frac{x}{c}\right)^{2 d}, \\
& R_{11}=2 d+b d+\frac{b(1-b)}{x^{2}}, \\
& R_{22}=R_{33}=\left(\frac{1+b}{x^{2}}-d\right)\left(\frac{x}{K}\right)^{2}\left(\frac{x}{c}\right)^{2 d} . \\
& R_{i}^{i}
\end{aligned}
$$

$$
R=2\left(\frac{1+b+b^{2}}{x^{2}}-2 d-b d\right)\left(\frac{x}{c}\right)^{2 d} .
$$

$T_{i j}$

$$
\begin{aligned}
\kappa T_{00} & =\left(2 d-\frac{1}{x^{2}}\right)\left(\frac{x}{c}\right)^{2 d}\left(\frac{x}{a}\right)^{2 b}, \\
\kappa T_{11} & =(1+2 b) \frac{1}{x^{2}}, \\
\kappa T_{22} & =\kappa T_{33}=\left[-d(1+b)+\frac{b^{2}}{x^{2}}\right]\left(\frac{x}{c}\right)^{2 d}\left(\frac{x}{K}\right)^{2} . \\
T_{i}^{i} & \\
T & =-2\left(\frac{1+b+b^{2}}{x^{2}}-2 d-b d\right)\left(\frac{x}{c}\right)^{2 d} .
\end{aligned}
$$

Case 4.

$$
\begin{aligned}
d s^{2}=e^{2 x / a} d t^{2}-d x^{2}-e^{2 x / b}\left(d y^{2}+d z^{2}\right), & (a \neq b \neq 0) . \\
R_{i j k l} & \\
R_{0101} & =-\frac{1}{a^{2}} e^{2 x / a}, \\
R_{0202} & =R_{0303}=-\frac{1}{a b} e^{2 x / a+2 x / b}, \\
R_{1212} & =R_{1313}=\frac{1}{b^{2}} e^{2 x / b}, \\
R_{2323} & =\frac{1}{b^{2}} e^{4 x / b} \cdot \\
R_{i j} \quad & \\
R_{00} & =\frac{1}{a}\left(\frac{1}{a}+\frac{2}{b}\right) e^{2 x / a}, \\
R_{11} & =-\left(\frac{1}{a^{2}}+\frac{2}{b^{2}}\right), \\
C_{13}^{2} & =1, \\
C_{12}^{3} & =-1, \\
R_{22} & =R_{33}=\frac{1}{b}\left(\frac{1}{a}+\frac{2}{b}\right) e^{2 x / b} . \\
R_{i}^{i} \quad & =C_{24}^{2}=C_{34}^{3}=-\frac{1}{a} . \\
a^{2} &
\end{aligned}
$$


$T_{i j}$

$$
\begin{aligned}
& \kappa T_{00}=-\frac{3}{b^{2}} e^{2 x / a}, \\
& \kappa T_{11}=\frac{1}{b}\left(\frac{1}{a}+\frac{2}{b}\right), \\
& \kappa T_{22}=\kappa T_{33}=\left(\frac{1}{a^{2}}+\frac{1}{a b}+\frac{1}{b^{2}}\right) e^{2 x / b} .
\end{aligned}
$$

$T^{i}{ }_{i}$

$$
T=-2\left(\frac{1}{a^{2}}+\frac{2}{a b}+\frac{3}{b^{2}}\right) .
$$

Case 5.

$$
\begin{array}{r}
d s^{2}=d t^{2}-e^{2 t / b} d x^{2}-e^{2 t / a}\left(d y^{2}+d z^{2}\right), \\
(a \neq b \neq 0) .
\end{array}
$$

The structure constants of the corresponding Lie algebra are the same as for (301).

$$
\begin{aligned}
R_{i j k l} & \\
R_{0101} & =\frac{1}{b^{2}} e^{2 t / b}, \\
R_{0202} & =R_{0303}=\frac{1}{a^{2}} e^{2 t / a}, \\
R_{1212} & =R_{1313}=-\frac{1}{a b} e^{2 t / a+2 t / b}, \\
R_{2323} & =-\frac{1}{a^{2}} e^{4 t / a} . \\
R_{i j} \quad & =-\left(\frac{2}{a^{2}}+\frac{1}{b^{2}}\right), \\
R_{11} & =\frac{1}{b}\left(\frac{2}{a}+\frac{1}{b}\right) e^{2 t / b}, \\
R_{22} & =R_{33}=\frac{1}{a}\left(\frac{2}{a}+\frac{1}{b}\right) e^{2 t / a} . \\
R_{i}^{i} \quad R & =-2\left(\frac{3}{a^{2}}+\frac{2}{a b}+\frac{1}{b^{2}}\right) .
\end{aligned}
$$

$T_{i j}$

$$
\begin{aligned}
& \kappa T_{00}=\frac{1}{a}\left(\frac{1}{a}+\frac{2}{b}\right), \\
& \kappa T_{11}=-\frac{3}{a^{2}} e^{2 t / b}, \\
& \kappa T_{22}=\kappa T_{33}=-\left(\frac{1}{a^{2}}+\frac{1}{a b}+\frac{1}{b^{2}}\right) e^{2 t / a} .
\end{aligned}
$$

$T^{i}{ }_{i}$

$$
T=2\left(\frac{3}{a^{2}}+\frac{2}{a b}+\frac{1}{b^{2}}\right) .
$$

Case 6.

(i) $d s^{2}$

$$
=d t^{2}
$$

$$
-\left(\frac{t}{a}\right)^{2}\left[d x^{2}+\sinh ^{2}\left(\frac{x}{b}\right)\left(d y^{2}+d z^{2}\right)\right]
$$

(ii) $d s^{2}$

$$
=d t^{2}
$$

$$
\begin{array}{r}
-\left(\frac{t}{a}\right)^{2}\left[d x^{2}+\cosh ^{2}\left(\frac{x}{b}\right)\left(d y^{2}+d z^{2}\right)\right], \\
(a \neq b \neq 0) .
\end{array}
$$

$C_{i j}^{k}$

$$
\begin{aligned}
& C_{13}^{2}=C_{\widehat{01}}^{\hat{2}}=C_{\widehat{0} \hat{2}}^{\hat{0}}=1, \\
& C_{12}^{3}=C_{\widehat{1} \hat{2}}^{\hat{1}}=-1 .
\end{aligned}
$$

$$
R_{i j k l}
$$

For (i)

$$
\begin{aligned}
& R_{1212}=R_{1313}=\left(\frac{1}{b^{2}}-\frac{1}{a^{2}}\right)\left(\frac{t}{a}\right)^{2} \sinh ^{2}\left(\frac{x}{b}\right), \\
& R_{2323}=\left(\frac{t}{a}\right)^{2} \sinh ^{2}\left(\frac{x}{b}\right) \\
& \cdot\left[\frac{1}{b^{2}} \cosh ^{2}\left(\frac{x}{b}\right)-\frac{1}{a^{2}} \sinh ^{2}\left(\frac{x}{b}\right)\right] .
\end{aligned}
$$

\section{For (ii)}

$$
\begin{aligned}
R_{1212} & =R_{1313}=\left(\frac{1}{b^{2}}-\frac{1}{a^{2}}\right)\left(\frac{t}{a}\right)^{2} \cosh ^{2}\left(\frac{x}{b}\right), \\
R_{2323} & =\left(\frac{t}{a}\right)^{2} \cosh ^{2}\left(\frac{x}{b}\right) \\
\cdot & {\left[\frac{1}{b^{2}} \sinh ^{2}\left(\frac{x}{b}\right)-\frac{1}{a^{2}} \cosh ^{2}\left(\frac{x}{b}\right)\right] . }
\end{aligned}
$$

$R_{i j}$

For (i)

$$
\begin{aligned}
& R_{11}=2\left(\frac{1}{a^{2}}-\frac{1}{b^{2}}\right), \\
& R_{22}=R_{33}=\left(\frac{2}{a^{2}}-\frac{1}{b^{2}}\right) \sinh ^{2}\left(\frac{x}{b}\right)-\frac{1}{b^{2}} \cosh ^{2}\left(\frac{x}{b}\right) .
\end{aligned}
$$


For (ii)

$$
\begin{aligned}
R_{11}= & 2\left(\frac{1}{a^{2}}-\frac{1}{b^{2}}\right), \\
R_{22}= & R_{33}=\left(\frac{2}{a^{2}}-\frac{1}{b^{2}}\right) \cosh ^{2}\left(\frac{x}{b}\right)+\frac{1}{b^{2}} \sinh ^{2}\left(\frac{x}{b}\right) . \\
& R_{i}^{i}
\end{aligned}
$$$$
\text { For (i) }
$$$$
R=\left(\frac{a}{t}\right)^{2}\left[\frac{2}{b^{2}}\left\{2+\operatorname{coth}^{2}\left(\frac{x}{b}\right)\right\}-\frac{6}{a^{2}}\right] .
$$$$
\text { For (ii) }
$$$$
R=\left(\frac{a}{t}\right)^{2}\left[\frac{2}{b^{2}}\left\{2+\tanh ^{2}\left(\frac{x}{b}\right)\right\}-\frac{6}{a^{2}}\right] .
$$

$T_{i j}$

For (i)

$$
\begin{aligned}
& \kappa T_{00}=\frac{1}{t^{2}}\left[3-\left(\frac{a}{b}\right)^{2}\left\{2+\operatorname{coth}^{2}\left(\frac{x}{b}\right)\right\}\right], \\
& \kappa T_{11}=-\frac{1}{a^{2}}+\frac{1}{b^{2}} \operatorname{coth}^{2}\left(\frac{x}{b}\right), \\
& \kappa T_{22}=\kappa T_{33}=\left(\frac{1}{b^{2}}-\frac{1}{a^{2}}\right) \sinh ^{2}\left(\frac{x}{b}\right) .
\end{aligned}
$$$$
\text { For (ii) }
$$$$
\kappa T_{00}=\frac{1}{t^{2}}\left[3-\left(\frac{a}{b}\right)^{2}\left\{2+\tanh ^{2}\left(\frac{x}{b}\right)\right\}\right],
$$$$
\kappa T_{11}=-\frac{1}{a^{2}}+\frac{1}{b^{2}} \tanh ^{2}\left(\frac{x}{b}\right),
$$$$
\kappa T_{22}=\kappa T_{33}=\left(\frac{1}{b^{2}}-\frac{1}{a^{2}}\right) \cosh ^{2}\left(\frac{x}{b}\right) .
$$$$
T^{i}{ }_{i}
$$

For (i)

$$
\begin{aligned}
& T=-\left(\frac{a}{t}\right)^{2}\left[\frac{2}{b^{2}}\left\{2+\operatorname{coth}^{2}\left(\frac{x}{b}\right)\right\}-\frac{6}{a^{2}}\right] . \\
& \text { For (ii) } \\
& T=-\left(\frac{a}{t}\right)^{2}\left[\frac{2}{b^{2}}\left\{2+\tanh ^{2}\left(\frac{x}{b}\right)\right\}-\frac{6}{a^{2}}\right] .
\end{aligned}
$$

Case 7.

(i) $d s^{2}$

$$
\begin{aligned}
= & \left(\frac{x}{a}\right)^{2}\left[d t^{2}-\sinh ^{2}\left(\frac{t}{b}\right)\left(d y^{2}+d z^{2}\right)\right] \\
& -d x^{2},
\end{aligned}
$$

(ii) $d s^{2}$

$$
\begin{aligned}
= & \left(\frac{x}{a}\right)^{2}\left[d t^{2}-\cosh ^{2}\left(\frac{t}{b}\right)\left(d y^{2}+d z^{2}\right)\right] \\
& -d x^{2}, \quad(a \neq b \neq 0) .
\end{aligned}
$$

The structure constants of the corresponding Lie algebra are the same as for (314).

$$
\begin{aligned}
& R_{i j k l} \\
& \text { For (i) } \\
& R_{0202}=R_{0303}=\left(\frac{1}{b^{2}}-\frac{1}{a^{2}}\right)\left(\frac{x}{a}\right)^{2} \sinh ^{2}\left(\frac{t}{b}\right), \\
& R_{2323}=\left(\frac{x}{a}\right)^{2} \sinh ^{2}\left(\frac{t}{b}\right) \\
& \cdot\left[\frac{1}{a^{2}} \sinh ^{2}\left(\frac{t}{b}\right)-\frac{1}{b^{2}} \cosh ^{2}\left(\frac{t}{b}\right)\right] \text {. } \\
& \text { For (ii) } \\
& R_{0202}=R_{0303}=\left(\frac{1}{b^{2}}-\frac{1}{a^{2}}\right)\left(\frac{x}{a}\right)^{2} \cosh ^{2}\left(\frac{t}{b}\right), \\
& R_{2323}=\left(\frac{x}{a}\right)^{2} \cosh ^{2}\left(\frac{t}{b}\right) \\
& \cdot\left[\frac{1}{a^{2}} \cosh ^{2}\left(\frac{t}{b}\right)-\frac{1}{b^{2}} \sinh ^{2}\left(\frac{t}{b}\right)\right] . \\
& R_{i j} \\
& \text { For (i) } \\
& R_{00}=2\left(\frac{1}{a^{2}}-\frac{1}{b^{2}}\right) \text {, } \\
& R_{22}=R_{33}=\left(\frac{1}{b^{2}}-\frac{2}{a^{2}}\right) \sinh ^{2}\left(\frac{t}{b}\right)+\frac{1}{b^{2}} \cosh ^{2}\left(\frac{t}{b}\right) . \\
& \text { For (ii) } \\
& R_{00}=2\left(\frac{1}{a^{2}}-\frac{1}{b^{2}}\right) \text {, } \\
& R_{22}=R_{33}=\left(\frac{1}{b^{2}}-\frac{2}{a^{2}}\right) \cosh ^{2}\left(\frac{t}{b}\right)+\frac{1}{b^{2}} \sinh ^{2}\left(\frac{t}{b}\right) . \\
& R_{i}^{i} \\
& \text { For (i) } \\
& R=\left(\frac{a}{x}\right)^{2}\left[\frac{6}{a^{2}}-\frac{2}{b^{2}}\left\{2+\operatorname{coth}^{2}\left(\frac{t}{b}\right)\right\}\right] . \\
& \text { For (ii) } \\
& R=\left(\frac{a}{x}\right)^{2}\left[\frac{6}{a^{2}}-\frac{2}{b^{2}}\left\{2+\tanh ^{2}\left(\frac{t}{b}\right)\right\}\right] . \\
& T_{i j} \\
& \text { For (i) } \\
& \kappa T_{00}=-\frac{1}{a^{2}}+\frac{1}{b^{2}} \operatorname{coth}^{2}\left(\frac{t}{b}\right), \\
& \kappa T_{11}=\frac{1}{x^{2}}\left[3-\left(\frac{a}{b}\right)^{2}\left\{2+\operatorname{coth}^{2}\left(\frac{t}{b}\right)\right\}\right], \\
& \kappa T_{22}=\kappa T_{33}=\left(\frac{1}{a^{2}}-\frac{1}{b^{2}}\right) \sinh ^{2}\left(\frac{t}{b}\right) .
\end{aligned}
$$


TABLE 44: Conserved quantity corresponding to the Noether symmetry.

\begin{tabular}{ll}
\hline Generator & Conserved quantity: $\varphi\left(s, q^{i}, \dot{q}^{i}\right)$ \\
\hline $\mathbf{X}_{0}=\partial_{t}$ & $-2 e^{2 \nu(x)} \dot{t}$ \\
\hline
\end{tabular}

TABLE 45: Conserved quantity corresponding to the Noether symmetry.

\begin{tabular}{ll}
\hline Generator & Conserved quantity: $\varphi\left(s, q^{i}, \dot{q}^{i}\right)$ \\
\hline $\mathbf{X}_{0}=\partial_{x}$ & $2 e^{2 \gamma(t, x)} \dot{x}$ \\
\hline
\end{tabular}

For (ii)

$$
\begin{aligned}
& \kappa T_{00}=-\frac{1}{a^{2}}+\frac{1}{b^{2}} \tanh ^{2}\left(\frac{t}{b}\right), \\
& \kappa T_{11}=\frac{1}{x^{2}}\left[3-\left(\frac{a}{b}\right)^{2}\left\{2+\tanh ^{2}\left(\frac{t}{b}\right)\right\}\right], \\
& \kappa T_{22}=\kappa T_{33}=\left(\frac{1}{a^{2}}-\frac{1}{b^{2}}\right) \cosh ^{2}\left(\frac{t}{b}\right) . \\
& T_{i}^{i}
\end{aligned}
$$$$
\text { For (i) }
$$$$
T=-\left(\frac{a}{x}\right)^{2}\left[\frac{6}{a^{2}}-\frac{2}{b^{2}}\left\{2+\operatorname{coth}^{2}\left(\frac{t}{b}\right)\right\}\right] .
$$

For (ii)

$$
T=-\left(\frac{a}{x}\right)^{2}\left[\frac{6}{a^{2}}-\frac{2}{b^{2}}\left\{2+\tanh ^{2}\left(\frac{t}{b}\right)\right\}\right] .
$$

4.7. Five Noether Symmetries. In this section, we present the metrics that admit five Noether symmetries, out of which four symmetries (minimal set) are given in (7) and the fifth generator along with its conserved form is given in Tables 44-46, respectively. The structure constants of the associated Lie algebra and the nonzero components of relevant tensors are also calculated.

Case 1.

$$
\begin{aligned}
& \text { (i) } d s^{2} \\
& \quad=e^{2 v(x)} d t^{2}-e^{2 \lambda(x)} d x^{2}-\left(\frac{x}{K}\right)^{2}\left(d y^{2}+d z^{2}\right), \\
& \text { (ii) } d s^{2}=e^{2 v(x)} d t^{2}-d x^{2}-e^{2 \mu(x)}\left(d y^{2}+d z^{2}\right), \\
& \text { (iii) } d s^{2}=e^{2 v(x)} d t^{2}-d x^{2}-e^{2 x / a}\left(d y^{2}+d z^{2}\right),
\end{aligned}
$$

where $a \neq 0$ and $K \neq 0$ are arbitrary constants and $\nu(x), \lambda(x)$, and $\mu(x)$ are arbitrary functions.

The structure constants of the corresponding Lie algebra are $C_{13}^{2}=1$ and $C_{12}^{3}=-1$.
TABLE 46: Noether symmetry and the corresponding conserved form.

\begin{tabular}{ll}
\hline Generator & $\begin{array}{l}\text { Conserved quantity: } \\
\varphi\left(s, q^{i}, \dot{q}^{i}\right)\end{array}$ \\
\hline (i) $\mathbf{X}_{0}=a \frac{\nu^{\prime \prime}}{\dot{\nu}^{\prime}} \partial_{t}-a \partial_{x}+y \partial_{y}+z \partial_{z}$ & $2 a\left(-e^{2 v(t, x)} \frac{\nu^{\prime \prime}}{\dot{\nu}^{\prime}} \dot{t}+\dot{x}\right)+$ \\
& $2 e^{2 x / a}(y \dot{y}+z \dot{z})$ \\
(ii) $\mathbf{X}_{0}=-a \partial_{t}+a \frac{\ddot{\lambda}}{\dot{\lambda}^{\prime}} \partial_{x}+y \partial_{y}+z \partial_{z}$ & $2 a\left(\dot{t}+e^{2 \lambda(t, x)} \frac{\ddot{\lambda}}{\dot{\lambda^{\prime}}} \dot{x}\right)+$ \\
& $2 e^{2 t / a}(y \dot{y}+z \dot{z})$ \\
\hline
\end{tabular}

$R_{i j k l}$

For (i)

$$
\begin{aligned}
& R_{0101}=-e^{2 \nu}\left(\nu^{\prime \prime}+\nu^{\prime 2}-\nu^{\prime} \lambda^{\prime}\right), \\
& R_{0202}=R_{0303}=-\left(\frac{x}{K^{2}}\right) \nu^{\prime} e^{2(\nu-\lambda)}, \\
& R_{1212}=R_{1313}=-\left(\frac{x}{K^{2}}\right) \lambda^{\prime}, \\
& R_{2323}=\left(\frac{x}{K^{2}}\right)^{2} e^{-2 \lambda} .
\end{aligned}
$$

For (ii)

$$
\begin{aligned}
& R_{0101}=-e^{2 \nu}\left(\nu^{\prime \prime}+\nu^{\prime 2}\right), \\
& R_{0202}=R_{0303}=-e^{2(\nu+\mu)} \nu^{\prime} \mu^{\prime}, \\
& R_{1212}=R_{1313}=e^{2 \mu}\left(\mu^{\prime \prime}+\mu^{\prime 2}\right), \\
& R_{2323}=\mu^{\prime 2} e^{4 \mu} .
\end{aligned}
$$

For (iii)

$$
\begin{aligned}
& R_{0101}=-e^{2 v}\left(v^{\prime \prime}+v^{\prime 2}\right), \\
& R_{0202}=R_{0303}=-\frac{1}{a} \nu^{\prime} e^{2(\nu+x / a)}, \\
& R_{1212}=R_{1313}=\frac{1}{a^{2}} e^{2 x / a}, \\
& R_{2323}=\frac{1}{a^{2}} e^{4 x / a} .
\end{aligned}
$$

$R_{i j}$

For (i)

$$
\begin{aligned}
& R_{00}=e^{2(\nu-\lambda)}\left[\nu^{\prime \prime}+\nu^{\prime 2}-\nu^{\prime}\left(\lambda^{\prime}-\frac{2}{x}\right)\right], \\
& R_{11}=-\left[\nu^{\prime \prime}+\nu^{\prime 2}-\lambda^{\prime}\left(\nu^{\prime}+\frac{2}{x}\right)\right] \\
& R_{22}=R_{33}=-\left(\nu^{\prime}-\lambda^{\prime}+\frac{1}{x}\right)\left(\frac{x}{K^{2}}\right) e^{-2 \lambda} .
\end{aligned}
$$


For (ii)

$$
\begin{aligned}
& R_{00}=e^{2 \nu}\left(\nu^{\prime \prime}+\nu^{\prime 2}+2 \nu^{\prime} \mu^{\prime}\right), \\
& R_{11}=-\left(\nu^{\prime \prime}+v^{\prime 2}+2 \mu^{\prime \prime}+2 \mu^{\prime 2}\right), \\
& R_{22}=R_{33}=-e^{2 \mu}\left(\mu^{\prime \prime}+\mu^{\prime 2}+\nu^{\prime} \mu^{\prime}\right) .
\end{aligned}
$$

For (iii)

$$
\begin{aligned}
& R_{00}=e^{2 v}\left(v^{\prime \prime}+v^{\prime 2}+\frac{2}{a} v^{\prime}\right), \\
& R_{11}=-\left(v^{\prime \prime}+v^{\prime 2}+\frac{2}{a^{2}}\right), \\
& R_{22}=R_{33}=-\frac{1}{a}\left(v^{\prime}+\frac{2}{a}\right) e^{2 x / a} .
\end{aligned}
$$

$R_{i}^{i}$

For (i)

$$
R=2\left[v^{\prime \prime}+\nu^{\prime 2}-v^{\prime} \lambda^{\prime}+\frac{2}{x}\left(\nu^{\prime}-\lambda^{\prime}\right)+\frac{1}{x^{2}}\right] e^{-2 \lambda}
$$

For (ii)

$$
R=2\left(2 \mu^{\prime \prime}+3 \mu^{\prime 2}+2 \nu^{\prime} \mu^{\prime}+\nu^{\prime \prime}+\nu^{\prime 2}\right) .
$$

For (iii)

$$
R=2\left[\frac{3}{a^{2}}+v^{\prime}\left(\frac{2}{a}+v^{\prime}\right)+v^{\prime \prime}\right] .
$$

$T_{i j}$

For (i)

$$
\begin{aligned}
\kappa T_{00} & =e^{2(\nu-\lambda)}\left(\frac{2 \lambda^{\prime}}{x}-\frac{1}{x^{2}}\right) \\
\kappa T_{11} & =\frac{2 \nu^{\prime}}{x}+\frac{1}{x^{2}} \\
\kappa T_{22} & =\kappa T_{33} \\
& =\left[\nu^{\prime \prime}+\nu^{\prime 2}-\nu^{\prime} \lambda^{\prime}+\frac{1}{x}\left(\nu^{\prime}-\lambda^{\prime}\right)\right]\left(\frac{x}{K}\right)^{2} e^{-2 \lambda} .
\end{aligned}
$$

For (ii)

$$
\begin{aligned}
& \kappa T_{00}=e^{2 \nu}\left(-2 \mu^{\prime \prime}-3 \mu^{\prime 2}\right), \\
& \kappa T_{11}=\mu^{\prime 2}+2 \nu^{\prime} \mu^{\prime}, \\
& \kappa T_{22}=\kappa T_{33}=e^{2 \mu}\left(\mu^{\prime \prime}+\mu^{\prime 2}+\nu^{\prime} \mu^{\prime}+\nu^{\prime \prime}+\nu^{\prime 2}\right) .
\end{aligned}
$$

For (iii)

$$
\begin{aligned}
& \kappa T_{00}=-\frac{3}{a^{2}} e^{2 v}, \\
& \kappa T_{11}=\frac{1}{a}\left(2 v^{\prime}+\frac{1}{a}\right), \\
& \kappa T_{22}=\kappa T_{33}=e^{2 v}\left[\frac{1}{a}\left(v^{\prime}+\frac{1}{a}\right)+v^{\prime \prime}+v^{\prime 2}\right] .
\end{aligned}
$$

$T_{i}^{i}$

For (i)

$T=-2\left[\nu^{\prime \prime}+\nu^{\prime 2}-v^{\prime} \lambda^{\prime}+\frac{2}{x}\left(\nu^{\prime}-\lambda^{\prime}\right)+\frac{1}{x^{2}}\right] e^{-2 \lambda}$.

For (ii)

$$
T=-2\left(2 \mu^{\prime \prime}+3 \mu^{\prime 2}+2 \nu^{\prime} \mu^{\prime}+\nu^{\prime \prime}+\nu^{\prime 2}\right) .
$$

For (iii)

$$
T=2\left(2 \ddot{\mu}+3 \dot{\mu}^{2}+2 \dot{\lambda} \dot{\mu}+\ddot{\lambda}+\dot{\lambda}^{2}\right) .
$$

Case 2.

$$
\begin{aligned}
& \text { (i) } d s^{2}=d t^{2}-e^{2 \lambda(t)} d x^{2}-e^{2 \mu(t)}\left(d y^{2}+d z^{2}\right), \\
& \text { (ii) } d s^{2}=d t^{2}-e^{2 \lambda(t)} d x^{2}-e^{2 t / a}\left(d y^{2}+d z^{2}\right),
\end{aligned}
$$

where $a \neq 0$ is an arbitrary constant and $\lambda(t)$ and $\mu(t)$ are arbitrary functions. The structure constants of the corresponding Lie algebra are the same as for (337).

$$
R_{i j k l}
$$

For (i)

$$
\begin{aligned}
& R_{0101}=e^{2 \lambda}\left(\ddot{\lambda}+\dot{\lambda}^{2}\right), \\
& R_{0202}=R_{0303}=e^{2 \mu}\left(\ddot{\mu}+\dot{\mu}^{2}\right), \\
& R_{1212}=R_{1313}=-e^{2(\lambda+\mu)} \dot{\lambda} \dot{\mu}, \\
& R_{2323}=-\dot{\mu}^{2} e^{4 \mu} .
\end{aligned}
$$

For (ii)

$$
\begin{aligned}
& R_{0101}=e^{2 \lambda}\left(\ddot{\lambda}+\dot{\lambda}^{2}\right), \\
& R_{0202}=R_{0303}=\frac{1}{a^{2}} e^{2 t / a}, \\
& R_{1212}=R_{1313}=-\frac{1}{a} \dot{\lambda} e^{2(\lambda+t / a)}, \\
& R_{2323}=-\frac{1}{a^{2}} e^{4 t / a} .
\end{aligned}
$$




$$
R_{i j}
$$

For (i)

$$
\begin{aligned}
& R_{00}=-\left(\ddot{\lambda}+\dot{\lambda}^{2}+2 \ddot{\mu}+2 \dot{\mu}^{2}\right), \\
& R_{11}=e^{2 \lambda}\left(\ddot{\lambda}+\dot{\lambda}^{2}+2 \dot{\lambda} \dot{\mu}\right), \\
& R_{22}=R_{33}=-e^{2 \mu}\left(\ddot{\mu}+\dot{\mu}^{2}+\dot{\lambda} \dot{\mu}\right) .
\end{aligned}
$$

For (ii)

$$
\begin{aligned}
& R_{00}=-\left(\ddot{\lambda}+\dot{\lambda}^{2}+\frac{2}{a^{2}}\right), \\
& R_{11}=e^{2 \lambda}\left(\ddot{\lambda}+\dot{\lambda}^{2}+\frac{2 \dot{\lambda}}{a}\right), \\
& R_{22}=R_{33}=-e^{2 t / a}\left(\frac{2}{a^{2}}+\frac{\dot{\lambda}}{a}\right) .
\end{aligned}
$$

$R_{i}^{i}$

For (i)

$$
R=-2\left(2 \ddot{\mu}+3 \dot{\mu}^{2}+2 \dot{\lambda} \dot{\mu}+\ddot{\lambda}+\dot{\lambda}^{2}\right) .
$$

For (ii)

$$
R=-2\left(\frac{3}{a^{2}}+\frac{2 \dot{\lambda}}{a}+\ddot{\lambda}+\dot{\lambda}^{2}\right) .
$$

$T_{i j}$

For (i)

$$
\begin{aligned}
& \kappa T_{00}=\dot{\mu}^{2}+2 \dot{\lambda} \dot{\mu}, \\
& \kappa T_{11}=e^{2 \lambda}\left(-2 \ddot{\mu}-3 \dot{\mu}^{2}\right), \\
& \kappa T_{22}=\kappa T_{33}=-e^{2 \mu}\left(\ddot{\mu}+\dot{\mu}^{2}+\dot{\lambda} \dot{\mu}+\ddot{\lambda}+\dot{\lambda}^{2}\right) .
\end{aligned}
$$

For (ii)

$$
\begin{aligned}
& \kappa T_{00}=\frac{1}{a^{2}}+\frac{2 \dot{\lambda}}{a}, \\
& \kappa T_{11}=-\frac{3}{a^{2}} e^{2 \lambda}, \\
& \kappa T_{22}=\kappa T_{33}=-e^{2 t / a}\left(\frac{1}{a^{2}}+\frac{\dot{\lambda}}{a}+\ddot{\lambda}+\dot{\lambda}^{2}\right) . \\
& T^{i}{ }_{i}
\end{aligned}
$$$$
\text { For (i) }
$$$$
T=2\left(2 \ddot{\mu}+3 \dot{\mu}^{2}+2 \dot{\lambda} \dot{\mu}+\ddot{\lambda}+\dot{\lambda}^{2}\right) .
$$

For (ii)

$$
T=2\left(\frac{3}{a^{2}}+\frac{2 \dot{\lambda}}{a}+\ddot{\lambda}+\dot{\lambda}^{2}\right) .
$$

Case 3.

$$
\begin{aligned}
& \text { (i) } d s^{2}=e^{2 \gamma(t, x)} d t^{2}-d x^{2}-e^{2 x / a}\left(d y^{2}+d z^{2}\right), \\
& \text { (ii) } d s^{2}=d t^{2}-e^{2 \lambda(t, x)} d x^{2}-e^{2 t / a}\left(d y^{2}+d z^{2}\right),
\end{aligned}
$$

where $a \neq 0$ is a real constant and $\nu(t, x)$ and $\lambda(t, x)$ denote arbitrary functions.

The structure constants of the corresponding Lie algebra are $C_{13}^{2}=1$ and $C_{12}^{3}=C_{02}^{2}=C_{03}^{3}=-1$. The nonzero components of the relevant tensors for (364)(i), (ii) are the same as the ones obtained for metrics (337)(iii) and (353)(ii), respectively.

4.8. Four Noether Symmetries. Finally, the metrics admitting the minimal set (7) of Noether symmetries are

$$
\begin{aligned}
& \text { (i) } d s^{2}=e^{2 v(t, x)} d t^{2}-d x^{2}-e^{2 \mu(x)}\left(d y^{2}+d z^{2}\right), \\
& \text { (ii) } d s^{2}=d t^{2}-e^{2 \lambda(t, x)} d x^{2}-e^{2 \mu(t)}\left(d y^{2}+d z^{2}\right),
\end{aligned}
$$

where $\nu(t, x), \lambda(t, x), \mu(t)$, and $\mu(x)$ are arbitrary functions. The structure constants of the corresponding Lie algebra for these metrics are $C_{13}^{2}=1$ and $C_{12}^{3}=-1$. The relevant tensors of these metrics are the same as the ones given for metrics (337)(ii) and (353)(i), respectively.

\section{Conclusion}

The Noether symmetries associated with the geodetic Lagrangian of general plane symmetric nonstatic space-times along with the conserved quantities corresponding to each symmetry, and the associated Lie algebras in terms of their structure constants and the relevant tensors of these metrics have been obtained. It turns out that general plane symmetric space-times can admit $4,5,6,7,8,9,11$, or 17 Noether symmetries. The maximum number of Noether symmetries (i.e., 17) appears only for the Minkowski space-time and 10 of these symmetries correspond to the generators of isometries associated with this metric. The maximal isometry group, in this case, is denoted by $G_{10}$. Other space-times that admit the maximal isometry group $G_{10}$ are de Sitter and anti-de Sitter space-times, which have a total of 11 Noether symmetries. Apart from these 10 isometries the only additional symmetry is $\mathbf{Y}_{0}=\partial_{s}$. This is the only Noether symmetry (other than isometries and general homotheties) that does not have a gauge term and corresponds to the constancy of the given Lagrangian along solutions, that is, geodesics. The Einstein and anti-Einstein metrics, space-times of Bertotti-Robinson type, and metrics given by (42), (49), (55), (62), (68), (75), (81), (88), (94), (101), (108), (115), (122), (129), and (135) admit 9 Noether symmetries. There are 8 metrics, given by (141), (148), (154), (161), (168), (180), (187), and (193), which 
admit 8 Noether symmetries. The ones admitting 7 Noether symmetries are given by (205), (212), (218), (225), (232), (239), (246), and (263). There are 7 metrics that admit 6 Noether symmetries and are given by (280), (287), (294), (301), (308), (314), and (326). The metrics that admit 5 Noether symmetries are given by (337), (353), and (364). Metric (365) admits the minimal set of Noether symmetries (i.e., 4).

Metrics (101), (108), (141), (154), (161), (168), (180), (225), (294), and (326)(ii) have essential singularities at $x=0$. For metric (161), the scalar curvature is $R=0$; therefore, we have calculated the second curvature invariant $R_{2}=$ $R_{k l}^{i j} R_{i j}^{k l}=12 b^{4} / x^{8}$ to check the singularity. For metrics (168)(i) and (225) the scalar curvature $R$ becomes undefined at $x=0$ provided $b>-1$. For metrics (168)(ii) and (294), scalar curvature $R$ becomes undefined at $x=0$ provided $b<1$ and $d<1$, respectively. Metrics (115), (148), and (187) are nonstatic analogues of (108), (141), and (180), respectively. These nonstatic metrics together with (314)(ii) have essential singularities at $t=0$. Metric (193)(ii) has essential singularity at $b t \pm a x=0$. Metrics (246)(ii) and (263)(ii) have essential singularity at $x / b=n \pi$ and $t / b=n \pi$ for $n=0,1,2, \ldots$, respectively. Metrics (314)(i) and (326)(i) have essential singularity at $t=0, x / b=n \pi$ and $x=0$, and $t / b=n \pi$, where $n=0,1,2, \ldots$, respectively.

Finally, we want to stress the fact that the Noether symmetries of the geodetic Lagrangian are either elements of the Killing or isometry Lie algebra of the underlying Lorentzian manifold (which were denoted by $\mathbf{X}_{i}$ ) or can be interpreted (at least locally) as elements of the projective algebra of the extended space $\left(s, q^{i}\right)$ of variables on which the geodesic equations depend, namely, the parameter $s$ and the coordinates $q^{i}$. In fact, all nonisometry symmetry generators $\mathbf{Y}_{i}$ are dependent on the affine parameter $s$ [39].

\section{Conflicts of Interest}

The authors declare that there are no conflicts of interest.

\section{Acknowledgments}

The third author was partially supported by the Research Project (ID-PRY: 4863 and ID-PPTA: 5027) of the Faculty of Sciences, Pontificia Universidad Javeriana, Bogotá, Colombia. The publication of this work was financed by Vicerrectoría de Investigación (ID-PRY: 7929, ID-PPTA: 8159), Pontificia Universidad Javeriana, Bogotá, Colombia.

\section{References}

[1] L. P. Eisenhart, Continuous Groups of Transformations, Princeton University Press, 1933.

[2] D. Kramer, H. Stephani, E. Herlt, and M. MacCallum, Exact Solutions of Einstein's Field Equations, Cambridge University Press, Cambridge, UK, 1980.

[3] T. Feroze, F. M. Mahomed, and A. Qadir, "The connection between isometries and symmetries of geodesic equations of underlying spaces," Nonlinear Dynamics, vol. 45, no. 1, pp. 6574, 2006.
[4] Y. Bozhkov and I. L. Freire, "Special conformal groups of a Riemannian manifold and Lie point symmetries of the nonlinear Poisson equation," Journal of Differential Equations, vol. 249, pp. 872-913, 2010.

[5] A. Aslam and A. Qadir, "Noether symmetries of the areaminimizing lagrangian," Journal of Applied Mathematics, vol. 2012, Article ID 532690, 14 pages, 2012.

[6] A. Paliathanasis and M. Tsamparlis, "The geometric origin of Lie point symmetries of the Schrodinger equation and the Klein-Gordon equations," International Journal of Geometric Methods in Modern Physics, vol. 11, no. 4, Article ID 1450037, 17 pages, 2014.

[7] A. H. Bokhari and A. Qadir, "Symmetries of static, spherically symmetric spacetimes," Journal of Mathematical Physics, vol. 28, no. 5, pp. 1019-1022, 1987.

[8] M. Ziad, Spherically Symmetric Spacetimes [Ph.D. thesis], Quaid-i-Azam University, Islamabad, 1990.

[9] A. Qadir and M. Ziad, "The classification of spherically symmetric spacetimes," II Nuovo Cimento B, vol. 110, no. 3, pp. 317334, 1995.

[10] T. B. Farid, A. Qadir, and M. Ziad, "The classification of static plane symmetric spacetimes according to their Ricci collineations," Journal of Mathematical Physics, vol. 36, no. 10, pp. 5812$5828,1995$.

[11] A. H. Bokhari and A. R. Kashif, "Curvature collineations of some static spherically symmetric spacetimes," Journal of Mathematical Physics, vol. 37, no. 7, pp. 3498-3504, 1996.

[12] D. Ahmed and M. Ziad, "Homothetic motions of spherically symmetric spacetimes," Journal of Mathematical Physics, vol. 38, no. 5, pp. 2547-2552, 1997.

[13] T. Feroze, A. Qadir, and M. Ziad, "The classification of plane symmetric spacetimes by isometries," Journal of Mathematical Physics, vol. 42, no. 10, pp. 4947-4955, 2001.

[14] M. Ziad and S. Ehsan, "Classification of plane symmetric lorentzian manifolds according to their homotheties and Classification of plane symmetric lorentzian manifolds according to their homotheties and metrics," IL Nuovo Cimento, vol. 123, no. 1, pp. 71-83, 2008.

[15] A. Qadir and K. Saifullah, "Comments on matter collineations of plane symmetric, cylindrically symmetric and spherically symmetric spacetimes," Journal of Mathematical Physics, vol. 45, no. 11, pp. 4191-4192, 2004.

[16] M. Sharif, "Classification of static plane symmetric spacetimes according to their matter collineations," Journal of Mathematical Physics, vol. 45, no. 4, pp. 1518-1531, 2004.

[17] M. Tsamparlis and A. Paliathanasis, "Lie and Noether symmetries of geodesic equations and collineations," General Relativity and Gravitation, vol. 42, no. 12, pp. 2957-2980, 2010.

[18] M. Tsamparlis, A. Paliathanasis, and L. Karpathopoulos, "Autonomous three-dimensional Newtonian systems which admit Lie and Noether point symmetries," Journal of Physics. A. Mathematical and Theoretical, vol. 45, no. 27, Article ID 275201, 12 pages, 2012.

[19] H. Azad, A. Y. Al-Dweik, R. Ghanam, and M. T. Mustafa, "Symmetry analysis of wave equation on static spherically symmetric spacetimes with higher symmetries," Journal of Mathematical Physics, vol. 54, no. 6, Article ID 063509, 23 pages, 2013.

[20] M. Tsamparlis, "Geometrization of Lie and Noether symmetries with applications in Cosmology," Journal of Physics: Conference Series, vol. 453, no. 1, Article ID 012020, 2013. 
[21] M. T. Mustafa and A. Y. Al-Dweik, "Noether symmetries and conservation laws of wave equation on static spherically symmetric spacetimes with higher symmetries," Communications in Nonlinear Science and Numerical Simulation, vol. 23, no. 1-3, pp. 141-152, 2015.

[22] M. Tsamparlis, A. Paliathanasis, and A. Qadir, "Noether symmetries and isometries of the minimal surface Lagrangian under constant volume in a Riemannian space," International Journal of Geometric Methods in Modern Physics, vol. 12, no. 1, Article ID 1550003, 10 pages, 2015.

[23] M. Tsamparlis, "Geometrization of Lie and Noether symmetries and applications," International Journal of Modern Physics: Conference Series, vol. 38, Article ID 1560078, 20 pages, 2015.

[24] E. Buhe, G. Bluman, and A. H. Kara, "Conservation laws for some systems of nonlinear PDEs via the symmetry/adjoint symmetry pair method," Journal of Mathematical Analysis and Applications, vol. 436, no. 1, pp. 94-103, 2016.

[25] H. Stephani and M. MacCallum, Differential Equations-Their Solution Using Symmetries, Cambridge University Press, Cambridge, UK, 1989.

[26] P. E. Hydon, Symmetry Methods for Differential Equations: A Beginner's Guide, Cambridge University Press, Cambridge, UK, 2000.

[27] N. H. Ibragimov, Elementary Lie Group Analysis and Ordinary Differential Equations, Wiley, Chichester, UK, 1999.

[28] E. Noether, "Invariante variations probleme," Nachr. d. König. Gesellsch. d. Wiss. zu Göttingen, Math-Phys. Klasse, pp. 235-257, 1918, (see Transport Theory and Statistical Physics, vol. 1, no. 3, pp. 186-207, 1971 for an English translation).

[29] P. J. Olver, Applications of Lie Groups to Differential Equations, Springer Science \& Business Media, New York, NY, USA, 2000.

[30] M. Tsamparlis and A. Paliathanasis, "The geometric nature of Lie and Noether symmetries," General Relativity and Gravitation, vol. 43, no. 6, pp. 1861-1881, 2011.

[31] G. S. Hall, Symmetries and Curvature Structure in General Relativity, World Scientific, Singapore, 2004.

[32] M. Ziad and S. Ehsan, "Classification of plane symmetric lorentzian manifolds according to their homotheties and metrics," II Nuovo Cimento B, vol. 123, no. 1, pp. 71-83, 2008.

[33] F. Ali and T. Feroze, "Classification of plane symmetric static spacetimes according to their noether symmetries," International Journal of Theoretical Physics, vol. 52, no. 9, pp. 3329-3342, 2013.

[34] B. Jamil and T. Feroze, "Classification of a class of plane symmetric static spacetimes according to the noether symmetries," in Proceedings of the Thirteenth Marcel Grossmann Meeting on General Relativity, vol. 1, pp. 1840-1843, 2015.

[35] I. Hussain, F. M. Mahomed, and A. Qadir, "Approximate Noether symmetries of the geodesic equations for the chargedKerr spacetime and rescaling of energy," General Relativity and Gravitation, vol. 41, no. 10, pp. 2399-2414, 2009.

[36] W.-H. Steeb, Ontinuous Symmetries, Lie Algebras, Differential Equations and Computer Algebra, World Scientific Publishing Co Inc, 2007.

[37] A. M. Ahmad, A. H. Bokhari, and F. D. Zaman, "Symmetries, conservation laws and wave equation on the Milne metric," Journal of Applied Mathematics, vol. 2012, Article ID 153817, 7 pages, 2012.

[38] J. B. Griffiths and J. Podolský, Exact Spacetimes in Einstein's General Relativity, Cambridge University Press, Cambridge, UK, 2009.
[39] T. Feroze and F. Ali, "Corrigendum to "Noether symmetries and conserved quantities for spaces with a section of zero curvature"," Journal of Geometry and Physics, vol. 80, pp. 8889, 2014, Journal of Geometry and Physics, vol. 61, pp. 658-662, 2011. 


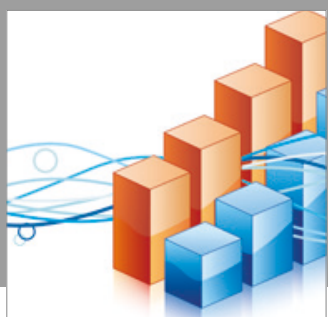

Advances in

Operations Research

vatersals

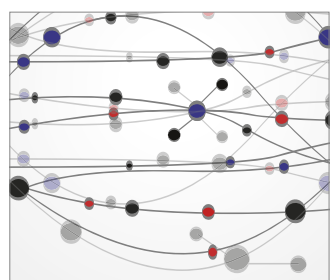

\section{The Scientific} World Journal
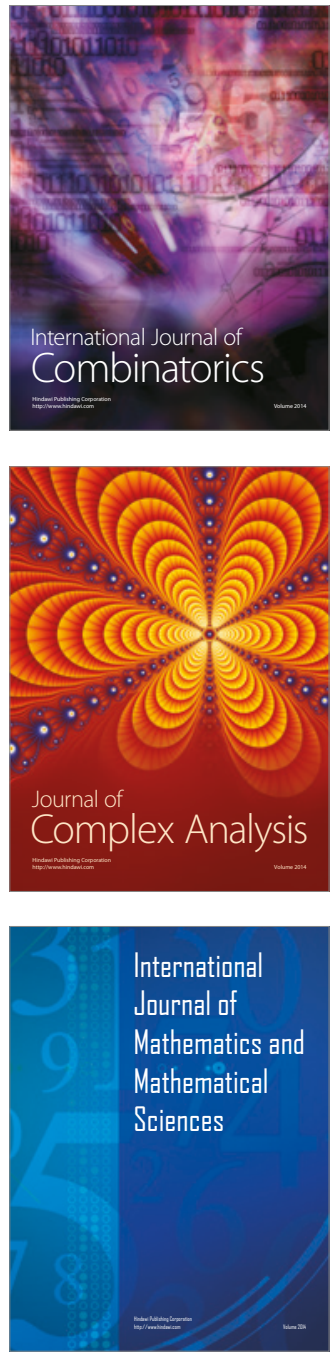
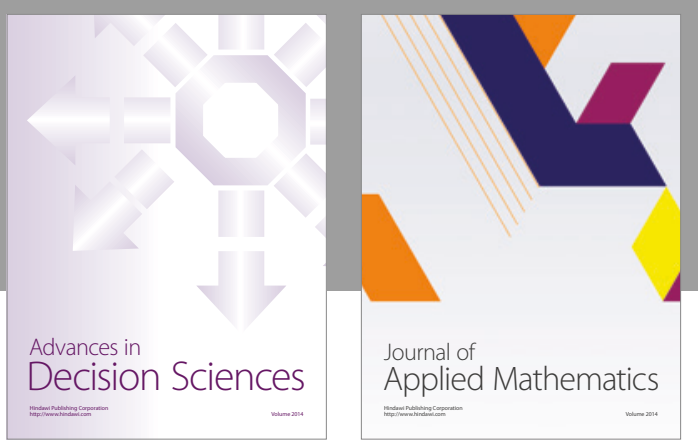

Algebra

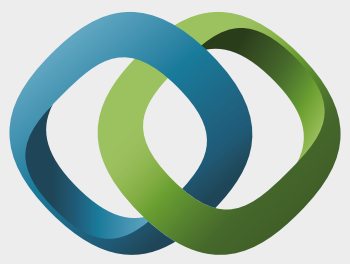

\section{Hindawi}

Submit your manuscripts at

https://www.hindawi.com
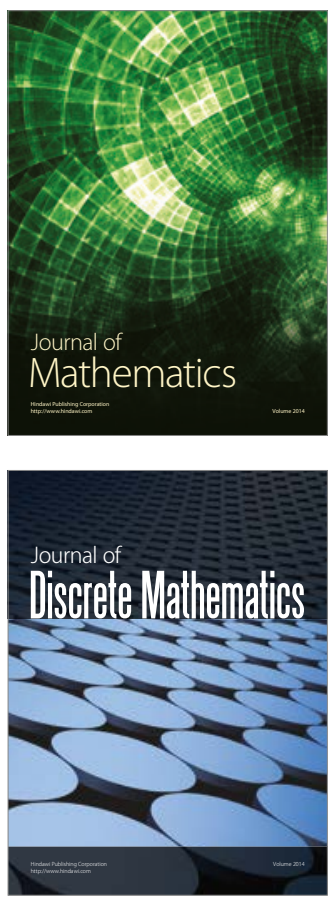

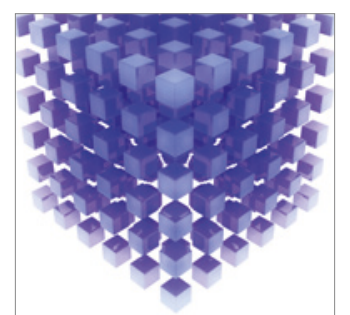

Mathematical Problems in Engineering
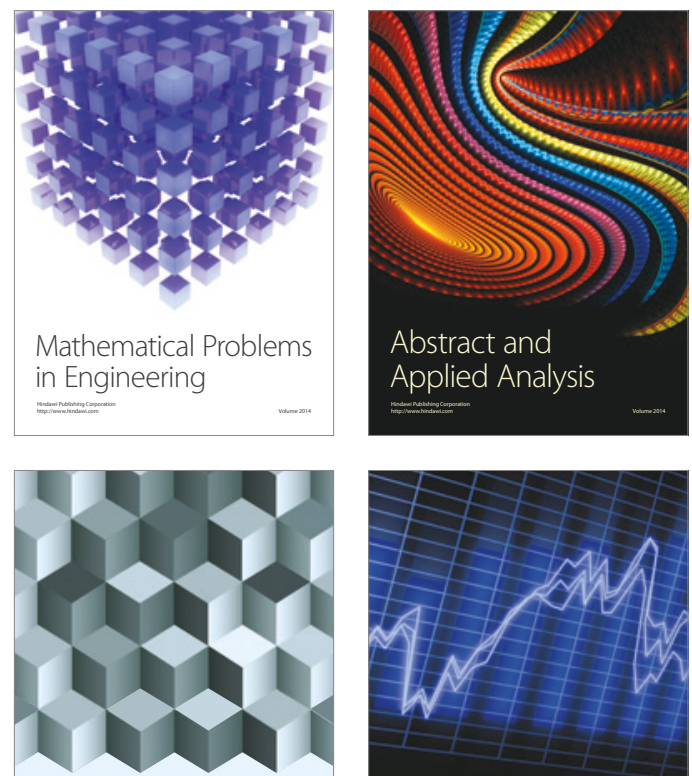

Journal of

Function Spaces

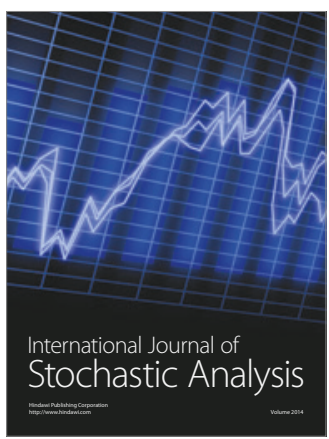

Probability and Statistics
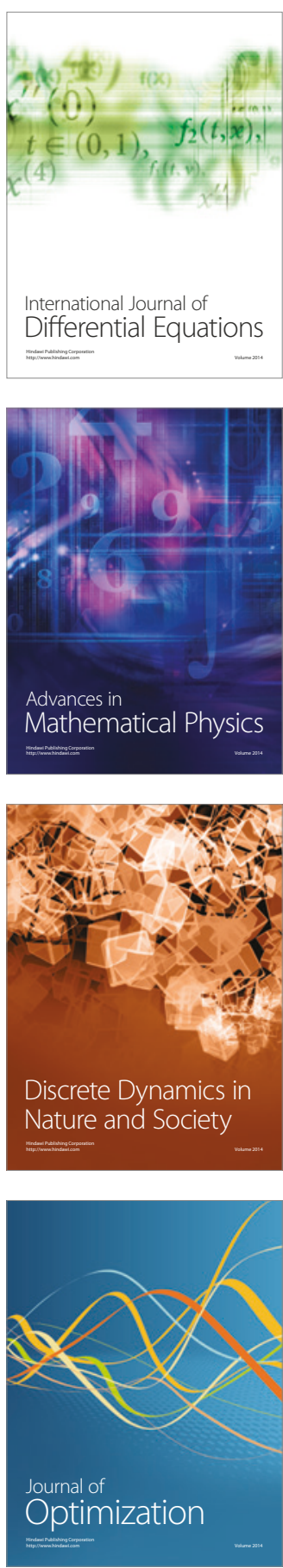